\title{
Catalytic Asymmetric Conjugate Addition of Carboxylic Acids via oxa-Michael Reaction of Peroxy-hemiacetals followed by Kornblum DeLaMare Fragmentation
}

Biswajit Parhi, Sanjay Maity and Prasanta Ghorai*

Department of Chemistry, Indian Institute of Science Education and Research Bhopal,

Bhopal By-pass Road, Bhauri, Bhopal-462066, India.

Email: pghorai@iiserb.ac.in

Contents:

Pages

General Experimental Procedures

(E)-2-(4-(4-Methoxyphenyl)-4-oxobut-2-en-1-yl)benzaldehyde (1c)

(E)-2-(4-(4-Chlorophenyl)-4-oxobut-2-en-1-yl)benzaldehyde (1d)

(E)-2-(4-(4-Bromophenyl)-4-oxobut-2-en-1-yl)benzaldehyde (1e)

(E)-2-(4-(4-Nitrophenyl)-4-oxobut-2-en-1-yl)benzaldehyde (1h)

(E)-2-(4-(Furan-2-yl)-4-oxobut-2-en-1-yl)benzaldehyde (1i)

(E)-2-(4-Oxo-4-(thiophen-2-yl)but-2-en-1-yl)benzaldehyde (1j)

(E)-5-Bromo-2-(4-(4-fluorophenyl)-4-oxobut-2-en-1-yl)benzaldehyde (1k)

Representative synthetic procedure for 1-Isochromanones:

Details of Optimization of Reaction condition:

3-(2-Oxo-2-phenylethyl)isochroman-1-one (3a)

3-(2-Oxo-2-(p-tolyl)ethyl)isochroman-1-one (3b)

3-(2-(4-Methoxyphenyl)-2-oxoethyl)isochroman-1-one (3c)

3-(2-(4-Chlorophenyl)-2-oxoethyl)isochroman-1-one (3d)

3-(2-(4-Bromophenyl)-2-oxoethyl)isochroman-1-one (3e)

3-(2-(4-Iodophenyl)-2-oxoethyl)isochroman-1-one (3f)

3-(2-(4-Fluorophenyl)-2-oxoethyl)isochroman-1-one (3g)

3-(2-(4-Nitrophenyl)-2-oxoethyl)isochroman-1-one (3h)

3-(2-(Furan-2-yl)-2-oxoethyl)isochroman-1-one (3i)

3-(2-Oxo-2-(thiophen-2-yl)ethyl)isochroman-1-one (3j)

7-Methyl-3-(2-oxo-2-(p-tolyl)ethyl)isochroman-1-one (3k)

7-Bromo-3-(2-(4-fluorophenyl)-2-oxoethyl)isochroman-1-one (3I)

(E)-2-(2-(3-(4-Bromophenyl)-3-oxoprop-1-en-1-yl)-4-methylphenyl)acetaldehyde (4b) 13

(E)-2-(2-(3-(4-Bromophenyl)-3-oxoprop-1-en-1-yl)-6-fluorophenyl)acetaldehyde (4d) 13

(E)-2-(2-(3-(4-Bromophenyl)-3-oxoprop-1-en-1-yl)-5-fluorophenyl)acetaldehyde (4e) 13

Representative synthetic procedure for 3-Isochromanones: $\quad 14$

1-(2-(4-Bromophenyl)-2-oxoethyl)isochroman-3-one (5a) 14

1-(2-(4-Bromophenyl)-2-oxoethyl)-7-methylisochroman-3-one (5b) 14

1-(2-(4-Bromophenyl)-2-oxoethyl)-6-chloroisochroman-3-one (5c) 15

1-(2-(4-Bromophenyl)-2-oxoethyl)-6-fluoroisochroman-3-one (5d) 15

(E)-2-(3-(2-Bromophenyl)-3-oxoprop-1-en-1-yl)benzaldehyde (6f) 16

(E)-2-(3-(4-Iodophenyl)-3-oxoprop-1-en-1-yl)benzaldehyde (6g) 16

(E)-2-(3-(3-Nitrophenyl)-3-oxoprop-1-en-1-yl)benzaldehyde (6k) 16

(E)-5-Methoxy-2-(3-oxo-3-(p-tolyl)prop-1-en-1-yl)benzaldehyde (6o) 16

(E)-5-Bromo-2-(3-(4-bromophenyl)-3-oxoprop-1-en-1-yl)benzaldehyde (6s) 17

(E)-2-(3-(4-Bromophenyl)-3-oxoprop-1-en-1-yl)-6-fluorobenzaldehyde (6u) 17

(E)-2-Fluoro-6-(3-oxo-3-(p-tolyl)prop-1-en-1-yl)benzaldehyde (6v) 17

(E)-1-(3-(4-Bromophenyl)-3-oxoprop-1-en-1-yl)-2-naphthaldehyde (6w) 18

(E)-2-(3-oxopent-1-en-1-yl)benzaldehyde (6x) 18 
Representative synthetic procedure for Phthalides: 18

3-(2-Oxo-2-phenylethyl)isobenzofuran-1(3H)-one (7a) 19

3-(2-Oxo-2-(p-tolyl)ethyl)isobenzofuran-1(3H)-one (7b) 19

3-(2-(4-Methoxyphenyl)-2-oxoethyl)isobenzofuran-1(3H)-one (7c) 20

3-(2-(4-Chlorophenyl)-2-oxoethyl)isobenzofuran-1(3H)-one (7d) 20

3-(2-(4-Bromophenyl)-2-oxoethyl)isobenzofuran-1(3H)-one (7e) 21

3-(2-(2-Bromophenyl)-2-oxoethyl)isobenzofuran-1(3H)-one (7f) 21

3-(2-(4-Iodophenyl)-2-oxoethyl)isobenzofuran-1(3H)-one (7g) 21

3-(2-(4-Fluorophenyl)-2-oxoethyl)isobenzofuran-1(3H)-one (7h) 22

3-(2-(2,4-Difluorophenyl)-2-oxoethyl)isobenzofuran-1(3H)-one (7i) 22

3-(2-(4-Nitrophenyl)-2-oxoethyl)isobenzofuran-1(3H)-one (7j) 23

3-(2-(3-Nitrophenyl)-2-oxoethyl)isobenzofuran-1(3H)-one (7k) 23

3-(2-(Furan-2-yl)-2-oxoethyl)isobenzofuran-1(3H)-one (7l) 24

3-(2-Oxo-2-(thiophen-2-yl)ethyl)isobenzofuran-1(3H)-one (7m) 24

3-(2-(4-Bromophenyl)-2-oxoethyl)-6-methoxyisobenzofuran-1(3H)-one (7n) 24

6-Methoxy-3-(2-oxo-2-(p-tolyl)ethyl)isobenzofuran-1(3H)-one (7o) 25

6-(Benzyloxy)-3-(2-(4-bromophenyl)-2-oxoethyl)-5-methoxyisobenzofuran-1(3H)-one (7p)

3-(2-(4-Bromophenyl)-2-oxoethyl)-5-methylisobenzofuran-1(3H)-one (7q) 26

3-(2-(4-Bromophenyl)-2-oxoethyl)-6-chloroisobenzofuran-1(3H)-one (7r) 26

6-Bromo-3-(2-(4-bromophenyl)-2-oxoethyl)isobenzofuran-1(3H)-one (7s) 27

3-(2-(4-Bromophenyl)-2-oxoethyl)-6-fluoroisobenzofuran-1(3H)-one (7t) 27

3-(2-(4-Bromophenyl)-2-oxoethyl)-7-fluoroisobenzofuran-1(3H)-one (7u) 27

7-Fluoro-3-(2-oxo-2-(p-tolyl)ethyl)isobenzofuran-1(3H)-one (7v) 28

1-(2-(4-Bromophenyl)-2-oxoethyl)naphtho[1,2-c]furan-3(1H)-one (7w) 28

3-(2-Oxobutyl)isobenzofuran-1(3H)-one (7x) 29

Synthetic Procedure for (E)-6-Oxo-6-phenylhex-4-enal/ (E)-6-Oxo-6-(p-tolyl)hex-4-enal: 29

(E)-6-Oxo-6-(p-tolyl)hex-4-enal (8a) 30

(E)-6-(4-Methoxyphenyl)-6-oxohex-4-enal (8b) 30

(E)-6-(Furan-2-yl)-6-oxohex-4-enal (8e) 30

(E)-6-Oxo-6-(thiophen-2-yl)hex-4-enal (8f) 30

(E)-6-Oxohept-4-enal (8g) 31

Representative synthetic procedure for $\gamma$-Lactones:

5-(2-Oxo-2-(p-tolyl)ethyl)dihydrofuran-2(3H)-one (9a) 31

5-(2-(4-Methoxyphenyl)-2-oxoethyl)dihydrofuran-2(3H)-one (9b) 32

5-(2-(4-Bromophenyl)-2-oxoethyl)dihydrofuran-2(3H)-one $(\mathbf{9 c}) \quad 32$

5-(2-(4-Iodophenyl)-2-oxoethyl)dihydrofuran-2(3H)-one (9d) 33

5-(2-(Furan-2-yl)-2-oxoethyl)dihydrofuran-2(3H)-one (9e) 33

5-(2-Oxo-2-(thiophen-2-yl)ethyl)dihydrofuran-2(3H)-one (9f) 33

5-(2-Oxopropyl)dihydrofuran-2(3H)-one (9g) 34

Reaction of in situ generated ortho-carboxylic acid containing chalcone 35

Decomposition Study: $\quad 37$

Gram scale synthesis: $\quad 42$

Determination of absolute configuration: $\quad 44$

References: $\quad 45$ 


\section{General Methods:}

All reagents and solvents were used as supplied commercially. Analytical thin-layer chromatography (TLC) were performed on $0.2 \mathrm{~mm}$ coated Science silica gel (EM 60-F254) plates purchased from Merck, Germany. Visualization was accomplished with UV light (254 $\mathrm{nm}$ ) and exposure to either ethanolic phosphomolybdic acid (PMA), anisaldehyde or $\mathrm{KMnO}_{4}$ solution, $\mathrm{CeSO}_{4}+$ ammoniu phosphomolybdate $+10 \% \mathrm{H}_{2} \mathrm{SO}_{4}$ followed by heating. Melting points are uncorrected. ${ }^{1} \mathrm{H}$ NMR spectra were acquired on a Bruker AVANCE (at $400 \mathrm{MHz}$ ) and chemical shifts are reported relative to the residual solvent peak. Only the variable temperature ${ }^{1} \mathrm{H}$ NMR experiments were performed on a Bruker AVANCE at $500 \mathrm{MHz}$ instrument. ${ }^{13} \mathrm{C}$ NMR spectra were acquired on Bruker AVANCE (at $100 \mathrm{MHz}$ ) and chemical shifts are reported in ppm relative to the residual solvent peak. Unless noted, NMR spectra were acquired in $\mathrm{CDCl}_{3}$; individual peaks are reported as: multiplicity ( $\mathrm{s}=$ singlet, $\mathrm{d}=$ doublet, $\mathrm{t}=$ triplet, $\mathrm{q}=$ quartet, $\mathrm{m}=$ multiplet), integration, coupling constant in Hz. All IR spectra were obtained as neat films and selected absorbances are reported in cm-1. Highresolution data were acquired using BrukerDaltonicsMicroTOF-Q-II Mass Spectrometer in $\mathrm{MeOH}$ as solvent or using Agilent GCQTOF Mass Spectrometer.

Representative Synthetic procedure for synthesis of Substrates 1a-k, see: ref. 2 Experimental Data for 1a-b, 1f-g, 1k, see: ref. 2

(E)-2-(4-(4-Methoxyphenyl)-4-oxobut-2-en-1-yl)benzaldehyde (1c): $146 \mathrm{mg} ; 36 \%$ yield; $\mathrm{R}_{\mathrm{f}}=0.16$ (10:90 = EtOAc/n-Hexane); Yellow colour sticky liquid; FT-IR (neat): 3017, 2896, 2883, 1668, 1565, 1263, 1010, $980 \mathrm{~cm}^{-1} ;{ }^{1} \mathbf{H}$ NMR (400 MHz, $\left.\mathbf{C D C l}_{3}\right): \delta, 10.18(\mathrm{~s}, 1 \mathrm{H}), 8.06(\mathrm{~d}, J=8.9 \mathrm{~Hz}, 1 \mathrm{H}), 7.87(\mathrm{~d}$, $J=8.9 \mathrm{~Hz}, 2 \mathrm{H}), 7.56(\mathrm{td}, J=7.5,1.4 \mathrm{~Hz}, 1 \mathrm{H}), 7.46(\mathrm{t}, J=7.5 \mathrm{~Hz}$,

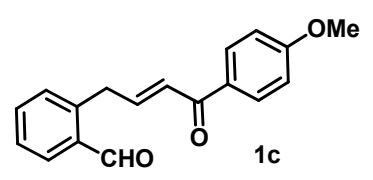
1H), 7.32 (d, $J=7.5 \mathrm{~Hz}, 1 \mathrm{H}), 6.98(\mathrm{~d}, J=8.9 \mathrm{~Hz}, 1 \mathrm{H}), 6.91$ (d, $J=8.9 \mathrm{~Hz}, 2 \mathrm{H}), 6.82$ (dd, $J=$ 9.1, 7.7 Hz, 1H), $4.07(\mathrm{dd}, J=6.5,1.0 \mathrm{~Hz}, 2 \mathrm{H}), 3.84(\mathrm{~s}, 3 \mathrm{H}) ;{ }^{13} \mathbf{C}$ NMR (101 MHz, CDCl $\mathbf{~}_{3}$ : $\delta, 192.6,188.9,163.4,145.8,140.1,134.6,134.1$, 133.7, 131.4, 130.9 (2C), 127.5, 126.9, 114.1, 113.8 (2C), 55.5, 35.7; HR-MS (ESI, m/z): Calculated for $\mathrm{C}_{18} \mathrm{H}_{17} \mathrm{O}_{3}\left([\mathrm{M}+\mathrm{H}]^{+}\right)$: 281.1172; Found: 281.1192.

(E)-2-(4-(4-Chlorophenyl)-4-oxobut-2-en-1-yl)benzaldehyde (1d): $65 \mathrm{mg} ; 54 \%$ yield; $\mathrm{R}_{\mathrm{f}}=$ 0.18 (20:80 = EtOAc/n-Hexane); Yellow colour semi-solid; FT-IR (neat): 3015, 2896, 2843, 2747, 2334, 1658, 1601, 1491, 1265, $1090 \mathrm{~cm}^{-1}$; ${ }^{1}$ H NMR (400

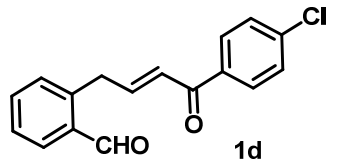


MHz, $\left.\mathbf{C D C l}_{3}\right): \delta, 10.14(\mathrm{~s}, 1 \mathrm{H}), 7.83(\mathrm{~d}, J=7.5 \mathrm{~Hz}, 1 \mathrm{H}), 7.78(\mathrm{~d}, J=8.5 \mathrm{~Hz}, 2 \mathrm{H}), 7.55(\mathrm{t}, J=$ $7.3 \mathrm{~Hz}, 1 \mathrm{H}), 7.46$ (t, $J=7.4 \mathrm{~Hz}, 1 \mathrm{H}), 7.38$ (d, $J=8.4 \mathrm{~Hz}, 2 \mathrm{H}), 7.30$ (d, $J=7.5 \mathrm{~Hz}, 1 \mathrm{H}), 7.15$ $(\mathrm{dd}, J=11.0,4.4 \mathrm{~Hz}, 1 \mathrm{H}), 6.75$ (d, $J=15.4 \mathrm{~Hz}, 1 \mathrm{H}), 4.07$ (d, $J=6.4 \mathrm{~Hz}, 2 \mathrm{H}) ;{ }^{13}$ C NMR (101 $\left.\mathbf{M H z}, \mathbf{C D C l}_{3}\right): \delta, 192.7,189.5,147.5,139.6,139.2,136.0,134.2,134.1,133.9,131.5,129.9$ (2C), 128.9 (2C), 127.6, 126.6, 35.9; HR-MS (ESI, m/z): Calculated for $\mathrm{C}_{17} \mathrm{H}_{14} \mathrm{ClO}_{2}$ $\left([\mathrm{M}+\mathrm{H}]^{+}\right): 285.0677$; Found: 285.0675 .

(E)-2-(4-(4-Bromophenyl)-4-oxobut-2-en-1-yl)benzaldehyde (1e): $60 \mathrm{mg} ; 55 \%$ yield; $\mathrm{R}_{\mathrm{f}}=$ $0.21(10: 90=$ EtOAc/n-Hexane); Yellow colour semi-solid; FTIR (neat): 3015, 2896, 2835, 2329, 1682, 1583, 1397, 1276, 1215, 1070, 1007, $700 \mathrm{~cm}^{-1} ;{ }^{1} \mathbf{H}$ NMR (400 MHz, $\left.\mathbf{C D C l}_{3}\right): \delta, 10.20(\mathrm{~s}$,

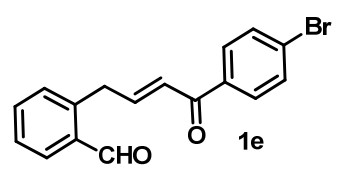
1H), $7.84(\mathrm{~d}, J=8.5 \mathrm{~Hz}, 2 \mathrm{H}), 7.76(\mathrm{~d}, J=7.6 \mathrm{~Hz}, 1 \mathrm{H}), 7.69$ (d, $J=8.5 \mathrm{~Hz}, 1 \mathrm{H}), 7.59$ (d, $J=$ $8.6 \mathrm{~Hz}, 2 \mathrm{H}), 7.54$ (dd, $J=6.8,4.7 \mathrm{~Hz}, 2 \mathrm{H}), 7.39$ (d, $J=6.7 \mathrm{~Hz}, 1 \mathrm{H}), 6.39$ (dt, $J=15.8,6.9$ $\mathrm{Hz}, 1 \mathrm{H}), 3.93(\mathrm{dd}, J=6.9,1.2 \mathrm{~Hz}, 2 \mathrm{H}) ;{ }^{13} \mathbf{C}$ NMR (101 MHz, $\left.\mathbf{C D C l}_{3}\right): \delta, 192.7,189.6$, 147.6, 139.4, 135.2, 134.1, 133.8, 132.3, 132.0 (2C), 130.3, 129.8 (2C), 127.7, 127.7, 126.6, 42.8; HR-MS (ESI, m/z): Calculated for $\mathrm{C}_{17} \mathrm{H}_{13} \mathrm{BrNaO}_{2}\left([\mathrm{M}+\mathrm{Na}]^{+}\right)$: 350.9991; Found: 350.9985 .

(E)-2-(4-(4-Nitrophenyl)-4-oxobut-2-en-1-yl)benzaldehyde (1h): $110 \mathrm{mg} ; 55 \%$ yield; $\mathrm{R}_{\mathrm{f}}=$ 0.20 (20:80 = EtOAc/n-Hexane); Brown colour oil; FT-IR (neat): 2989, 2874, 2843, 2364, 1673, 1511, 1338, 1110, 1026, $695 \mathrm{~cm}^{-1}$; ${ }^{1} \mathbf{H}$ NMR (400 MHz, CDCl $\left.\mathbf{~}_{3}\right): \delta, 10.13$ (s, 1H), 8.27 $(\mathrm{d}, J=8.8 \mathrm{~Hz}, 2 \mathrm{H}), 7.97(\mathrm{~d}, J=8.8 \mathrm{~Hz}, 2 \mathrm{H}), 7.84(\mathrm{dd}, J=7.5,1.2$ $\mathrm{Hz}, 1 \mathrm{H}), 7.58(\mathrm{td}, J=7.5,1.4 \mathrm{~Hz}, 1 \mathrm{H}), 7.50(\mathrm{td}, J=7.5,0.9 \mathrm{~Hz}$, 1H), 7.32 (d, $J=7.5 \mathrm{~Hz}, 1 \mathrm{H}), 7.23(\mathrm{~d}, J=7.5 \mathrm{~Hz}, 1 \mathrm{H}), 6.74(\mathrm{dt}, J$

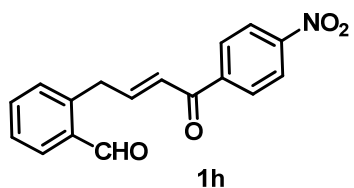
$=15.5,1.5 \mathrm{~Hz}, 1 \mathrm{H}), 4.10(\mathrm{dd}, J=6.5,1.2 \mathrm{~Hz}, 2 \mathrm{H}) ;{ }^{13} \mathbf{C}$ NMR (101 MHz, $\left.\mathbf{C D C l}_{3}\right): \delta, 192.8$, 189.4, 149.4, 142.6, 139.1, 134.8, 134.2, 133.9, 131.6, 129.5, 127.8, 126.6 (2C), 123.9, 123.8 (2C), 36.2; HR-MS (ESI, m/z): Calculated for $\mathrm{C}_{17} \mathrm{H}_{14} \mathrm{NO}_{4}\left([\mathrm{M}+\mathrm{H}]^{+}\right)$: 296.0917; Found: 296.0924 .

(E)-2-(4-(Furan-2-yl)-4-oxobut-2-en-1-yl)benzaldehyde (1i): $72 \mathrm{mg} ; 56 \%$ yield; $\mathrm{R}_{\mathrm{f}}=0.17$ (20:80 = EtOAc/n-Hexane); Yellow colour semi-solid; FT-IR (neat): 2949, 2927, 2857, 2821, 2364, 1677, 1603, 1454, 1287, 1033, $763 \mathrm{~cm}^{-1} ;{ }^{1}$ H NMR (400 MHz, $\left.\mathbf{C D C l}_{3}\right): \delta, 10.15(\mathrm{~s}, 1 \mathrm{H}), 7.83(\mathrm{dd}, J=7.5,1.1 \mathrm{~Hz}, 1 \mathrm{H}), 7.59-$ $7.52(\mathrm{~m}, 2 \mathrm{H}), 7.45$ (t, $J=7.2 \mathrm{~Hz}, 1 \mathrm{H}), 7.30$ (d, $J=8.0 \mathrm{~Hz}, 1 \mathrm{H}), 7.27$ -

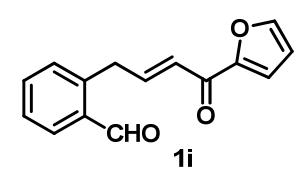
$7.21(\mathrm{~m}, 1 \mathrm{H}), 7.18(\mathrm{~d}, J=3.5 \mathrm{~Hz}, 1 \mathrm{H}), 6.72(\mathrm{~d}, J=15.5 \mathrm{~Hz}, 1 \mathrm{H}), 6.51(\mathrm{dd}, J=3.5,1.6 \mathrm{~Hz}$, 
1H), $4.06(\mathrm{dd}, J=6.6,1.1 \mathrm{~Hz}, 2 \mathrm{H}) ;{ }^{13} \mathbf{C}$ NMR (101 MHz, $\left.\mathbf{C D C l}_{3}\right): \delta, 192.6,177.9,153.1$, 146.66, 146.2, 139.8, 134.1, 133.9, 133.8, 131.4, 127.6, 126.1, 117.9, 112.4, 35.7; HR-MS (ESI, $\boldsymbol{m} / \mathbf{z})$ : Calculated for $\mathrm{C}_{15} \mathrm{H}_{13} \mathrm{O}_{3}\left([\mathrm{M}+\mathrm{H}]^{+}\right)$: 241.0859; Found: 241.0849.

(E)-2-(4-Oxo-4-(thiophen-2-yl)but-2-en-1-yl)benzaldehyde $(\mathbf{1 j}): 87 \mathrm{mg}, 61 \%$ yield; $\mathrm{R}_{\mathrm{f}}=$ 0.19 (20:80 = EtOAc/n-Hexane); Yellow colour semi-solid; FT-IR (neat): 2949, 2927, 2857 , 2821, 2364, 1677, 1603, 1454, 1287, 1033, $763 \mathrm{~cm}^{-1} ;{ }^{1}$ H NMR (400 MHz, $\left.\mathbf{C D C l}_{3}\right): \delta, 10.12(\mathrm{~s}, 1 \mathrm{H}), 7.80(\mathrm{~d}, J=7.5 \mathrm{~Hz}, 1 \mathrm{H}), 7.64(\mathrm{~d}, J=$ $3.7 \mathrm{~Hz}, 1 \mathrm{H}), 7.58(\mathrm{~d}, J=4.9 \mathrm{~Hz}, 1 \mathrm{H}), 7.52$ (t, $J=6.9 \mathrm{~Hz}, 1 \mathrm{H}), 7.42$ (t, $J$

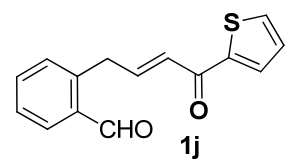
$=7.5 \mathrm{~Hz}, 1 \mathrm{H}), 7.28(\mathrm{~d}, J=7.5 \mathrm{~Hz}, 1 \mathrm{H}), 7.19(\mathrm{dt}, J=15.0,6.6 \mathrm{~Hz}, 1 \mathrm{H}), 7.06(\mathrm{t}, J=4.2 \mathrm{~Hz}$, 1H), $6.71(\mathrm{~d}, J=15.3 \mathrm{~Hz}, 1 \mathrm{H}), 4.04(\mathrm{~d}, J=6.6 \mathrm{~Hz}, 2 \mathrm{H}) ;{ }^{13} \mathbf{C}$ NMR (101 MHz, CDCl 3$): ~ \delta$, 192.6, 182.2, 146.1, 144.9, 139.8, 134.1, 134.0, 133.9, 133.8, 132.2, 131.4, 128.2, 127.6, 126.6, 35.6; HR-MS (ESI, m/z): Calculated for $\mathrm{C}_{15} \mathrm{H}_{13} \mathrm{O}_{2} \mathrm{~S}\left([\mathrm{M}+\mathrm{H}]^{+}\right)$: 257.0631; Found: 257.0646 .

(E)-5-Bromo-2-(4-(4-fluorophenyl)-4-oxobut-2-en-1-yl)benzaldehyde (11): $58 \mathrm{mg} ; 29 \%$ yield; $\mathrm{R}_{\mathrm{f}}=0.19$ (10:90 = EtOAc/n-Hexane); Yellow colour semi-solid; FT-IR (neat): 2996, 2856, 2843, 1673, 1525, 1474, 1260, 1125, 980, $750 \mathrm{~cm}^{-1} ;{ }^{1} \mathbf{H}$ NMR (400 MHz, $\left.\mathbf{C D C l}_{3}\right): \delta, 10.16(\mathrm{~s}, 1 \mathrm{H}), 7.89(\mathrm{dd}, J=8.3$, $5.6 \mathrm{~Hz}, 4 \mathrm{H}), 7.64-7.60(\mathrm{~m}, 2 \mathrm{H}), 7.47(\mathrm{~s}, 1 \mathrm{H}), 6.80(\mathrm{dd}, J=$

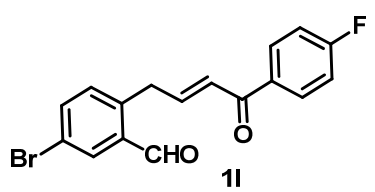
15.3, 1.5 Hz, 2H), $3.96(\mathrm{dd}, J=6.9,1.3 \mathrm{~Hz}, 2 \mathrm{H}) ;{ }^{13} \mathbf{C}$ NMR (101 MHz, $\left.\mathbf{C D C l}_{3}\right): \delta, 188.6$, $188.2,165.7$ (d, $J=254.6 \mathrm{~Hz}), 145.5,140.5,139.7,133.6,132.9,131.2$ (d, $J=7.7 \mathrm{~Hz}), 130.9$ $(\mathrm{d}, J=23.1 \mathrm{~Hz}), 128.4,127.2,124.9,123.8,115.9(\mathrm{~d}, J=21.9 \mathrm{~Hz}), 115.7(\mathrm{~d}, J=21.9 \mathrm{~Hz})$, 36.2; HR-MS (ESI, m/z): Calculated for $\mathrm{C}_{17} \mathrm{H}_{13} \mathrm{BrFO}_{2}\left([\mathrm{M}+\mathrm{H}]^{+}\right)$: 347.0077; Found: 347.0049 .

Optimization of the reaction conditions ${ }^{\text {a,b,c }}$ :

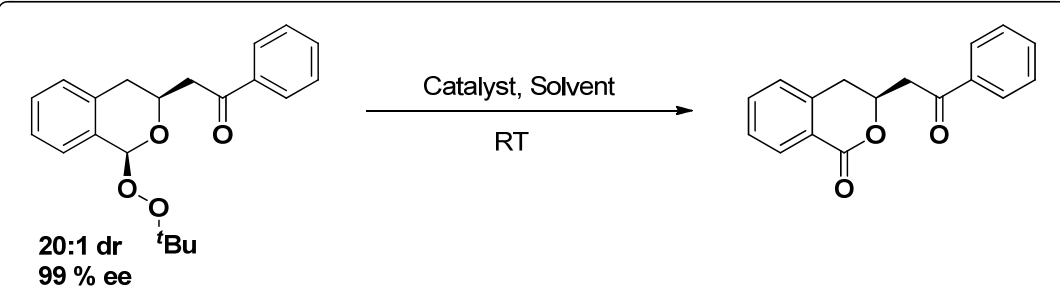

\begin{tabular}{|c|c|c|c|c|c|c|}
\hline Entry & Catalyst & Mol \% & Solvent & Time & $\%$ NMR Yield & $\%$ ee $^{\mathrm{e}}$ \\
\hline 1 & $\mathrm{Re}_{2} \mathrm{O}_{7}$ & 10 & Dichloromethane & $24 \mathrm{~h}$ & 5 & $\mathrm{ND}^{\mathrm{f}}$ \\
\hline
\end{tabular}




\begin{tabular}{|c|c|c|c|c|c|c|}
\hline 2 & $\mathrm{InCl}_{3}$ & 10 & Dichloromethane & $18 \mathrm{~h}$ & 8 & ND \\
\hline 3 & $\mathrm{ZnCl}_{2}$ & 10 & Dichloromethane & $18 \mathrm{~h}$ & 10 & ND \\
\hline 4 & $\mathrm{FeCl}_{3} \cdot 6 \mathrm{H}_{2} \mathrm{O}$ & 10 & Dichloromethane & $12 \mathrm{~h}$ & 94 & 97 \\
\hline 5 & $\mathrm{Bi}(\mathrm{OTf})_{3}$ & 10 & Dichloromethane & $24 \mathrm{~h}$ & 10 & ND \\
\hline 6 & $\mathrm{In}(\mathrm{OTf})_{3}$ & 10 & Dichloromethane & $24 \mathrm{~h}$ & 6 & ND \\
\hline 7 & $\mathrm{Et}_{3} \mathrm{~N}$ & 10 & Dichloromethane & $12 \mathrm{~h}$ & 31 & 0 \\
\hline 8 & $\mathrm{DBU}$ & 10 & Dichloromethane & $12 \mathrm{~h}$ & 100 & 0 \\
\hline 9 & $\mathrm{FeCl}_{3} \cdot 6 \mathrm{H}_{2} \mathrm{O}$ & 25 & Toluene & $12 \mathrm{~h}$ & 9 & 97 \\
\hline 10 & $\mathrm{FeCl}_{3} \cdot 6 \mathrm{H}_{2} \mathrm{O}$ & 25 & 1,2-DCE & $12 \mathrm{~h}$ & 63 & 97 \\
\hline 11 & $\mathrm{FeCl}_{3} \cdot 6 \mathrm{H}_{2} \mathrm{O}$ & 25 & Chloroform & $12 \mathrm{~h}$ & 90 & 97 \\
\hline 12 & $\mathrm{FeCl}_{3} \cdot 6 \mathrm{H}_{2} \mathrm{O}$ & 25 & Dichloromethane & $10 \mathrm{~h}$ & 100 & 97 \\
\hline 13 & $\mathrm{FeCl}_{3} \cdot 6 \mathrm{H}_{2} \mathrm{O}$ & 25 & Diethylether & $24 \mathrm{~h}$ & 0 & $\mathrm{ND}$ \\
\hline 14 & $\mathrm{FeCl}_{3} \cdot 6 \mathrm{H}_{2} \mathrm{O}$ & 25 & Acetonitrile & $24 \mathrm{~h}$ & 0 & $\mathrm{ND}$ \\
\hline 15 & $\mathrm{FeCl}_{3} \cdot 6 \mathrm{H}_{2} \mathrm{O}$ & 25 & Nitromethane & $24 \mathrm{~h}$ & 0 & $\mathrm{ND}$ \\
\hline 16 & $\mathrm{FeCl}_{3} \cdot 6 \mathrm{H}_{2} \mathrm{O}$ & 35 & Dichloromethane & $8 \mathrm{~h}$ & 100 & 97 \\
\hline
\end{tabular}

[a] All reactions are carried out at $0.03 \mathrm{mmol}$ scale. [b] The reaction mixture was filtered through a small plug of silica gel and was wahed with dichloromethane as solvent. [c] The initial selectivity of peroxide was 20:1 dr with $99 \%$ ee. [d] NMR yield was calculated by using $5 \mu L$ anisole as internal standard. [e] Enantiomeric excess was determined by HPLC analysis on a chiral stationary phase. [f] ND $=$ Not Determined

\section{Representative one-pot protocol for the synthesis of chiral 1-Isochromanones:}

\section{Step-I}

A solution of homo-chalcone 1 [1 equiv, $0.1 \mathrm{mmol}$ ] and catalyst $(5 \mathrm{~mol} \%)$ in toluene was taken in a small round bottom flask with a magnetic bar and was placed over a magnetic stirrer at room temperature. Then, $6 \mathrm{M}$ solution of TBHP (5 equiv) was added dropwise to the pre-mix solution. The reaction mixture was allowed to stir at room temperature for 3 to $7 \mathrm{~h}$. The reaction was stopped when all the starting material (homo-chalcone) were consumed (monitored by TLC). Finally, the reaction was filtered with a small plug of silica and washed with dichloromethane as solvent. The peroxy-acetal was directly taken for step-II after removing the solvent in vaccuo.

\section{Step-II}

The solution of peroxy-acetal (obtained from step-I) in dichloromethane was taken in a small round bottom flask with a bar magnet and was placed over a magnetic stirrer. The solid $\mathrm{FeCl}_{3} \cdot 6 \mathrm{H}_{2} \mathrm{O}(25 \mathrm{~mol} \%)$ was directly added to the flask and the reaction was allowed to stir at room temperature for overnight. Then the reaction was filtered with a small plug of silica gel 
and washed with dichloromethane as solvent. After the removal of solvent in vaccuo, the crude 1-Isochromanone was washed with n-hexane to get optically pure 1-Isochromanone.

All data were given after washing with n-hexane unless until mentioned:

3-(2-Oxo-2-phenylethyl)isochroman-1-one (3a): $9 \mathrm{mg} ; 42 \%$ yield; $\mathrm{R}_{\mathrm{f}}=0.25(20: 80=$ EtOAc/n-Hexane); White Solid; mp $128{ }^{0} \mathrm{C}$; FT-IR (neat): 3054, 2945, 2861, 2813, 2369, 1715, 1682, 1601, 1452, 1031, $761 \mathrm{~cm}^{-1}$; ${ }^{\mathbf{1}} \mathbf{H}$ NMR (400 MHz, $\left.\mathbf{C D C l}_{3}\right)$ : $\delta, 8.09(\mathrm{~d}, J=7.6 \mathrm{~Hz}, 2 \mathrm{H}), 8.01-7.88(\mathrm{~m}, 4 \mathrm{H}), 7.60(\mathrm{t}, J=7.4 \mathrm{~Hz}, 2 \mathrm{H})$, $7.54(\mathrm{td}, J=7.5,1.1 \mathrm{~Hz}, 2 \mathrm{H}), 7.48(\mathrm{t}, J=7.7 \mathrm{~Hz}, 4 \mathrm{H}), 7.40(\mathrm{t}, J=7.6$ $\mathrm{Hz}, 2 \mathrm{H}), 7.25$ (d, $J=7.5 \mathrm{~Hz}, 3 \mathrm{H}), 5.21$ (dddd, $J=11.2,8.0,4.7,3.3 \mathrm{~Hz}$,

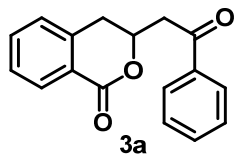
2H), $3.71(\mathrm{dd}, J=17.4,4.8 \mathrm{~Hz}, 2 \mathrm{H}), 3.36(\mathrm{dd}, J=17.4,8.0 \mathrm{~Hz}, 2 \mathrm{H}), 3.22(\mathrm{dd}, J=16.2,3.2$ $\mathrm{Hz}, 2 \mathrm{H}), 3.03(\mathrm{dd}, J=16.2,11.2 \mathrm{~Hz}, 2 \mathrm{H}) ;{ }^{13} \mathbf{C}$ NMR (101 MHz, $\left.\mathbf{C D C l}_{\mathbf{3}}\right): \delta, 196.2,165.2$, $138.7,136.4,133.9,133.7,130.3,128.8(2 \mathrm{C}), 128.1(2 \mathrm{C}), 127.8,127.6,124.9,74.8,43.5$, 33.2; HR-MS (ESI, m/z): Calculated for $\mathrm{C}_{17} \mathrm{H}_{14} \mathrm{NaO}_{3}\left([\mathrm{M}+\mathrm{Na}]^{+}\right)$: 289.0835; Found: $289.0861 ;[\alpha]_{\mathrm{D}}^{18}=-49.02\left(\mathrm{c}=0.5, \mathrm{CHCl}_{3},>99 \% \mathrm{ee}\right)$.

The enantiomeric ratio was determined by HPLC analysis using Diacel Chiralpak OD-3 column, $\mathrm{n}$-Hexane/2-Propanol $=75 / 25$, flow rate $=1.0 \mathrm{~mL} / \mathrm{min}, \lambda=254 \mathrm{~nm} ; \mathrm{t}_{\mathrm{R}}=13.4 \mathrm{~min}$ (major), $\mathrm{t}_{\mathrm{R}}=16.0 \mathrm{~min}$ (minor).

3-(2-Oxo-2-(p-tolyl)ethyl)isochroman-1-one (3b): (before wash $46 \mathrm{mg}$; 92\% yield; 96\% ee) $28 \mathrm{mg} ; 56 \%$ yield; $\mathrm{R}_{\mathrm{f}}=0.26\left(20: 80=\right.$ EtOAc/n-Hexane); White Solid; mp $118{ }^{0} \mathrm{C}$; FT-IR (neat): 3030, 2958, 2852, 1701, 1603, 1454, 1118, 917, $768 \mathrm{~cm}^{-1} ;{ }^{1} \mathbf{H}$ NMR (400 MHz, $\left.\mathbf{C D C l}_{3}\right): \delta, 8.08(\mathrm{~d}, J=7.7 \mathrm{~Hz}, 1 \mathrm{H}), 7.85(\mathrm{~d}, J=8.2$ $\mathrm{Hz}, 2 \mathrm{H}), 7.53(\mathrm{td}, J=7.5,1.0 \mathrm{~Hz}, 1 \mathrm{H}), 7.38$ (t, $J=7.6 \mathrm{~Hz}, 1 \mathrm{H}), 7.25$ (dd,

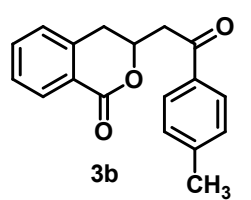
$J=11.2,7.2 \mathrm{~Hz}, 3 \mathrm{H}), 5.26-5.08(\mathrm{~m}, 1 \mathrm{H}), 3.67(\mathrm{dd}, J=17.3,4.8 \mathrm{~Hz}, 1 \mathrm{H}), 3.32(\mathrm{dd}, J=17.3$, $8.1 \mathrm{~Hz}, 1 \mathrm{H}), 3.21(\mathrm{dd}, J=16.2,3.1 \mathrm{~Hz}, 1 \mathrm{H}), 3.01$ (dd, $J=16.2,11.2 \mathrm{~Hz}, 1 \mathrm{H}), 2.40(\mathrm{~s}, 3 \mathrm{H})$; ${ }^{13}$ C NMR (101 MHz, $\left.\mathbf{C D C l}_{3}\right): \delta, 195.8,165.2,144.7,138.8,134.0,133.9,130.3,129.5(2 \mathrm{C})$, 128.2(2C), 127.8, 127.6, 125.0, 74.9, 43.3, 33.2, 21.7; HR-MS (ESI, m/z): Calculated for $\mathrm{C}_{18} \mathrm{H}_{17} \mathrm{O}_{3}\left([\mathrm{M}+\mathrm{H}]^{+}\right): 281.1172$; Found: 281.1167; $[\alpha]_{\mathrm{D}}^{22}=-141.6\left(\mathrm{c}=0.5, \mathrm{CHCl}_{3},>99 \%\right.$ ee $)$.

The enantiomeric ratio was determined by HPLC analysis using Diacel Chiralpak OD-3 column, $\mathrm{n}$-Hexane/2-Propanol $=85 / 15$, flow rate $=1.0 \mathrm{~mL} / \mathrm{min}, \lambda=254 \mathrm{~nm} ; \mathrm{t}_{\mathrm{R}}=17.2 \mathrm{~min}$ (major), $\mathrm{t}_{\mathrm{R}}=19.5 \mathrm{~min}$ (minor). 
3-(2-(4-Methoxyphenyl)-2-oxoethyl)isochroman-1-one (3c): (before wash $31 \mathrm{mg}$; 83\% yield; 96\% ee); $17 \mathrm{mg} ; 46 \%$ yield; $\mathrm{R}_{\mathrm{f}}=0.20(20: 80=\mathrm{EtOAc} / \mathrm{n}-$ Hexane); White Solid; mp $121{ }^{0}$ C; FT-IR (neat): 3019, 2989, 2896, 2835, 2356, 1721, 1675, 1241, 1169, $750 \mathrm{~cm}^{-1} ;{ }^{1}$ H NMR (400 MHz, $\left.\mathbf{C D C l}_{3}\right): \delta, 8.08(\mathrm{~d}, J=7.6 \mathrm{~Hz}, 2 \mathrm{H}), 7.94(\mathrm{~d}, J=8.9 \mathrm{~Hz}, 4 \mathrm{H}), 7.53(\mathrm{td}, J$

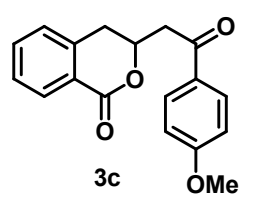
$=7.5,1.1 \mathrm{~Hz}, 2 \mathrm{H}), 7.39(\mathrm{t}, J=7.6 \mathrm{~Hz}, 2 \mathrm{H}), 7.24(\mathrm{t}, J=3.7 \mathrm{~Hz}, 3 \mathrm{H}), 6.94(\mathrm{~d}, J=8.9 \mathrm{~Hz}, 4 \mathrm{H})$, 5.18 (dddd, $J=11.3,8.0,4.7,3.3 \mathrm{~Hz}, 2 \mathrm{H}), 3.87$ (s, 6H), 3.65 (dd, $J=17.1,4.8 \mathrm{~Hz}, 2 \mathrm{H}), 3.26$ (ddd, $J=19.4,16.6,5.6 \mathrm{~Hz}, 4 \mathrm{H}), 3.02$ (dd, $J=16.2,11.2 \mathrm{~Hz}, 2 \mathrm{H}) ;{ }^{13} \mathbf{C}$ NMR (101 MHz, $\left.\mathbf{C D C l}_{3}\right): \delta, 194.7,165.2,164.0,138.8,133.9,130.5$ (2C), 130.3, 129.6, 127.8, 127.6, 125.0, 113.9 (2C), 75.1, 55.6, 43.1, 33.2; HR-MS (ESI, m/z): Calculated for $\mathrm{C}_{18} \mathrm{H}_{16} \mathrm{NaO}_{4}$ $\left([\mathrm{M}+\mathrm{Na}]^{+}\right): 319.0941$; Found: $319.0952 ;[\alpha]_{\mathrm{D}}^{23}=-98.04\left(\mathrm{c}=0.5, \mathrm{CHCl}_{3}, 97.3 \%\right.$ ee $)$.

The enantiomeric ratio was determined by HPLC analysis using Diacel Chiralpak OD-3 column, $\mathrm{n}$-Hexane/2-Propanol $=65 / 35$, flow rate $=1.0 \mathrm{~mL} / \mathrm{min}, \lambda=254 \mathrm{~nm} ; \mathrm{t}_{\mathrm{R}}=19.5 \mathrm{~min}$ (major), $\mathrm{t}_{\mathrm{R}}=24.4 \mathrm{~min}$ (minor).

3-(2-(4-Chlorophenyl)-2-oxoethyl)isochroman-1-one (3d): (before wash $35 \mathrm{mg}$; 79\% yield; $96 \%$ ee); $22 \mathrm{mg} ; 49 \%$ yield; $\mathrm{R}_{\mathrm{f}}=0.28$ (20:80 = EtOAc/n-Hexane); White Solid; mp $134{ }^{0} \mathrm{C}$; FT-IR (neat): 2971, 2861, 1701, 1688, 1298, $1090 \mathrm{~cm}^{-1}$; ${ }^{\mathbf{1}} \mathbf{H}$ NMR (400 MHz, CDCl $\left.\mathbf{3}\right): \delta$, $8.08(\mathrm{~d}, J=7.7 \mathrm{~Hz}, 1 \mathrm{H}), 7.90(\mathrm{~d}, J=8.5 \mathrm{~Hz}, 2 \mathrm{H}), 7.54$ (dd, $J=10.8,4.1$ $\mathrm{Hz}, 1 \mathrm{H}), 7.45$ (d, $J=8.5 \mathrm{~Hz}, 2 \mathrm{H}), 7.39$ (t, $J=7.6 \mathrm{~Hz}, 1 \mathrm{H}), 7.28-7.22$ $(\mathrm{m}, 1 \mathrm{H}), 5.18(\mathrm{tdd}, J=8.1,5.0,3.4 \mathrm{~Hz}, 1 \mathrm{H}), 3.66(\mathrm{dd}, J=17.4,5.1 \mathrm{~Hz}$, 1H), $3.30(\mathrm{dd}, J=17.4,7.6 \mathrm{~Hz}, 1 \mathrm{H}), 3.19(\mathrm{dd}, J=16.2,3.1 \mathrm{~Hz}, 1 \mathrm{H}), 3.03$

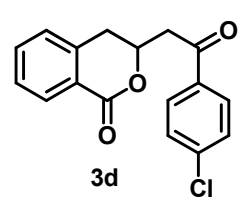

$(\mathrm{dd}, J=16.1,11.3 \mathrm{~Hz}, 1 \mathrm{H}) ;{ }^{13} \mathbf{C}$ NMR (101 MHz, $\left.\mathbf{C D C l}_{3}\right): \delta, 195.0,165.0,140.3,138.6$, $134.8,133.9,130.3,129.5(2 \mathrm{C}), 129.2(2 \mathrm{C}), 127.9,127.6,124.9,74.6,43.4,33.1$; HR-MS (ESI, m/z): Calculated for $\mathrm{C}_{17} \mathrm{H}_{13} \mathrm{ClNaO}_{3}\left([\mathrm{M}+\mathrm{Na}]^{+}\right)$: 323.0445; Found: $323.0456 ;[\alpha]_{\mathrm{D}}{ }^{21}=$ $91.5\left(\mathrm{c}=0.5, \mathrm{CHCl}_{3},>99 \%\right.$ ee $)$.

The enantiomeric ratio was determined by HPLC analysis using Diacel Chiralpak OD-3 column, $\mathrm{n}$-Hexane $/ 2$-Propanol $=75 / 25$, flow rate $=1.0 \mathrm{~mL} / \mathrm{min}, \lambda=254 \mathrm{~nm} ; \mathrm{t}_{\mathrm{R}}=16.4 \mathrm{~min}$ (major), $\mathrm{t}_{\mathrm{R}}=22.4 \mathrm{~min}$ (minor).

3-(2-(4-Bromophenyl)-2-oxoethyl)isochroman-1-one (3e): (before wash $58 \mathrm{mg}$; 90\% yield; 97\% ee); $31 \mathrm{mg} ; 48 \%$ yield; $\mathrm{R}_{\mathrm{f}}=0.26$ (20:80 = EtOAc/n-Hexane); Light Red Solid; mp 138 ${ }^{0} \mathrm{C}$; FT-IR (neat): 3024, 2997, 2883, 2839, 1690, 1585, 1296, 1125, 752

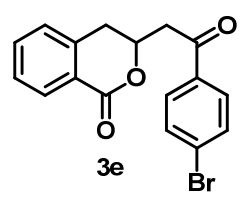


$\mathrm{cm}^{-1} ;{ }^{1} \mathbf{H}$ NMR (400 MHz, $\left.\mathbf{C D C l}_{3}\right): \delta, 8.08(\mathrm{~d}, J=7.7 \mathrm{~Hz}, 1 \mathrm{H}), 7.81(\mathrm{~d}, J=8.5 \mathrm{~Hz}, 2 \mathrm{H}), 7.62$ $(\mathrm{d}, J=8.5 \mathrm{~Hz}, 2 \mathrm{H}), 7.56-7.51(\mathrm{~m}, 1 \mathrm{H}), 7.39(\mathrm{t}, J=7.6 \mathrm{~Hz}, 1 \mathrm{H}), 7.28-7.22(\mathrm{~m}, 1 \mathrm{H}), 5.18$ $(\mathrm{dd}, J=8.0,2.9 \mathrm{~Hz}, 1 \mathrm{H}), 3.65(\mathrm{dd}, J=17.4,5.1 \mathrm{~Hz}, 1 \mathrm{H}), 3.29(\mathrm{dd}, J=17.4,7.5 \mathrm{~Hz}, 1 \mathrm{H})$, $3.18(\mathrm{dd}, J=16.2,3.2 \mathrm{~Hz}, 1 \mathrm{H}), 3.03(\mathrm{dd}, J=16.1,11.3 \mathrm{~Hz}, 1 \mathrm{H}) ;{ }^{13} \mathbf{C}$ NMR (101 MHz, $\left.\mathbf{C D C l}_{3}\right): \delta, 195.2,194.9,165.0,138.6,135.2,133.9,132.2(2 \mathrm{C}), 130.4,129.6(2 \mathrm{C}), 129.0$, 127.9, 127.6, 124.9, 74.6, 43.4, 33.1; HR-MS (ESI, m/z): Calculated for $\mathrm{C}_{17} \mathrm{H}_{14} \mathrm{BrO}_{3}$ $\left([\mathrm{M}+\mathrm{H}]^{+}\right): 345.0121 ;$ Found: $345.0105 ;[\alpha]_{\mathrm{D}}^{22}=-118.2\left(\mathrm{c}=0.5, \mathrm{CHCl}_{3}, 99 \%\right.$ ee $)$.

The enantiomeric ratio was determined by HPLC analysis using Diacel Chiralpak OD-3 column, $\mathrm{n}$-Hexane/2-Propanol $=75 / 25$, flow rate $=1.0 \mathrm{~mL} / \mathrm{min}, \lambda=254 \mathrm{~nm} ; \mathrm{t}_{\mathrm{R}}=20.4 \mathrm{~min}$ (major), $\mathrm{t}_{\mathrm{R}}=26.9 \min$ (minor).

3-(2-(4-Iodophenyl)-2-oxoethyl)isochroman-1-one (3f): (before wash $27 \mathrm{mg}$; 70\% yield; 94\% ee); $20 \mathrm{mg} ; 51 \%$ yield; $\mathrm{R}_{\mathrm{f}}=0.30$ (20:80 = EtOAc/n-Hexane); White Solid; mp $136{ }^{0} \mathrm{C}$; FT-IR (neat): 3010, 2967, 2879, 2839, 2356, 1719, 1688, 1246, 987, $761 \mathrm{~cm}^{-1} ;{ }^{1} \mathbf{H}$ NMR (400 MHz, $\left.\mathbf{C D C l}_{3}\right): \delta, 8.08(\mathrm{~d}, J=7.7 \mathrm{~Hz}, 2 \mathrm{H}), 7.85$ $(\mathrm{d}, J=8.4 \mathrm{~Hz}, 4 \mathrm{H}), 7.66(\mathrm{~d}, J=8.5 \mathrm{~Hz}, 4 \mathrm{H}), 7.57-7.51(\mathrm{~m}, 2 \mathrm{H}), 7.40(\mathrm{t}$, $J=7.6 \mathrm{~Hz}, 2 \mathrm{H}), 7.25(\mathrm{t}, J=3.7 \mathrm{~Hz}, 3 \mathrm{H}), 5.28-5.11(\mathrm{~m}, 2 \mathrm{H}), 3.64(\mathrm{dd}, J$

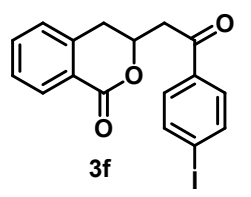
$=17.4,5.1 \mathrm{~Hz}, 2 \mathrm{H}), 3.28(\mathrm{dd}, J=17.4,7.6 \mathrm{~Hz}, 2 \mathrm{H}), 3.19(\mathrm{dd}, J=16.2,3.1 \mathrm{~Hz}, 2 \mathrm{H}), 3.03(\mathrm{dd}$, $J=16.1,11.3 \mathrm{~Hz}, 2 \mathrm{H}) ;{ }^{13} \mathbf{C}$ NMR (101 MHz, $\left.\mathbf{C D C l}_{3}\right): \delta, 195.5,165.0,138.6,138.2(2 \mathrm{C})$, 135.7, 133.9, 130.4, 129.5(2C), 127.9, 127.6, 124.9, 101.9, 74.6, 43.3, 33.1; HR-MS (ESI, $\mathbf{m} / \mathbf{z})$ : Calculated for $\mathrm{C}_{17} \mathrm{H}_{13} \mathrm{INaO}_{3}\left([\mathrm{M}+\mathrm{Na}]^{+}\right): 414.9802$; Found: $414.9788 ;[\alpha]_{\mathrm{D}}^{22}=-68.94(\mathrm{c}$ $=0.5, \mathrm{CHCl}_{3},>99 \%$ ee).

The enantiomeric ratio was determined by HPLC analysis using Diacel Chiralpak OD-3 column, $\mathrm{n}$-Hexane/2-Propanol $=75 / 25$, flow rate $=1.0 \mathrm{~mL} / \mathrm{min}, \lambda=254 \mathrm{~nm} ; \mathrm{t}_{\mathrm{R}}=27.5 \mathrm{~min}$ (major), $\mathrm{t}_{\mathrm{R}}=31.8 \min$ (minor).

3-(2-(4-Fluorophenyl)-2-oxoethyl)isochroman-1-one (3g): (before wash $30 \mathrm{mg}$; 94\% yield; $92 \%$ ee); $15 \mathrm{mg} ; 47 \%$ yield; $\mathrm{R}_{\mathrm{f}}=0.23$ (20:80 = EtOAc/n-Hexane); White Solid; mp $129{ }^{0} \mathrm{C}$; FT-IR (neat): 3026, 2993, 2852, 2273, 1699, 1603, 1452, 1228, 836, $750 \mathrm{~cm}^{-1} ;{ }^{1} \mathbf{H}$ NMR (400 MHz, $\left.\mathbf{C D C l}_{3}\right): \delta, 8.09(\mathrm{~d}, J=7.5 \mathrm{~Hz}, 2 \mathrm{H}), 8.03$ - $7.89(\mathrm{~m}, 4 \mathrm{H}), 7.54(\mathrm{td}, J=7.5,1.2 \mathrm{~Hz}, 2 \mathrm{H}), 7.40(\mathrm{t}, J=7.6 \mathrm{~Hz}, 2 \mathrm{H})$, $7.25(\mathrm{~d}, J=7.4 \mathrm{~Hz}, 3 \mathrm{H}), 7.21-7.07$ (m, 4H), 5.19 (dddd, $J=11.1,8.1$,

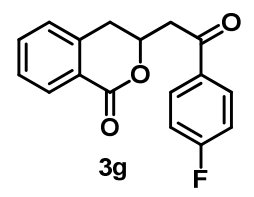
5.0, 3.3 Hz, 2H), $3.67(\mathrm{dd}, J=17.3,5.0 \mathrm{~Hz}, 2 \mathrm{H}), 3.31(\mathrm{dd}, J=17.3,7.7 \mathrm{~Hz}, 2 \mathrm{H}), 3.21(\mathrm{dd}, J$ $=16.2,3.2 \mathrm{~Hz}, 2 \mathrm{H}), 3.04(\mathrm{dd}, J=16.2,11.2 \mathrm{~Hz}, 2 \mathrm{H}) ;{ }^{13} \mathbf{C}$ NMR (101 MHz, CDCl $\left.\mathbf{3}\right): \delta$, 
194.6, 166.1(d, $J=256.0 \mathrm{~Hz}), 165.1,138.6,133.9,132.9$ (d, $J=3.0 \mathrm{~Hz}), 130.8$ (d, $J=9.4$ $\mathrm{Hz}), 130.4,127.9,127.6,124.9,116.1,115.9,74.7,43.4,33.2$; HR-MS (ESI, m/z): Calculated for $\mathrm{C}_{17} \mathrm{H}_{14} \mathrm{FO}_{3}\left([\mathrm{M}+\mathrm{H}]^{+}\right)$: 285.0921; Found: $285.0907 ;[\alpha]_{\mathrm{D}}^{21}=-62.085(\mathrm{c}=0.5$, $\mathrm{CHCl}_{3},>99 \%$ ee).

The enantiomeric ratio was determined by HPLC analysis using Diacel Chiralpak OD-3 column, $\mathrm{n}$-Hexane/2-Propanol $=75 / 25$, flow rate $=1.0 \mathrm{~mL} / \mathrm{min}, \lambda=254 \mathrm{~nm} ; \mathrm{t}_{\mathrm{R}}=12.2 \mathrm{~min}$ (major), $\mathrm{t}_{\mathrm{R}}=16.7 \mathrm{~min}$ (minor).

3-(2-(4-Nitrophenyl)-2-oxoethyl)isochroman-1-one (3h): (before wash $56 \mathrm{mg}$; 76\% yield; $88 \%$ ee); 30 mg; 40\% yield; $\mathrm{R}_{\mathrm{f}}=0.15$ (20:80 = EtOAc/n-Hexane); Brown Solid; $182{ }^{0} \mathrm{C}$; FTIR (neat): 2980, 2909, 2848, 1721, 1697, 1522, 1346, 1123, $851 \mathrm{~cm}^{-1}$; ${ }^{1}$ H NMR (400 MHz, $\left.\mathbf{C D C l}_{3}\right): \delta, 8.33(\mathrm{~d}, J=8.8 \mathrm{~Hz}, 2 \mathrm{H}), 8.11(\mathrm{dd}, J=$ 13.4, 8.4 Hz, 3H), $7.56(\mathrm{td}, J=7.5,1.1 \mathrm{~Hz}, 1 \mathrm{H}), 7.41(\mathrm{t}, J=7.6 \mathrm{~Hz}, 1 \mathrm{H})$, $7.27(\mathrm{~d}, J=7.6 \mathrm{~Hz}, 1 \mathrm{H}), 5.30-5.14(\mathrm{~m}, 1 \mathrm{H}), 3.74(\mathrm{dd}, J=17.5,5.6 \mathrm{~Hz}$,

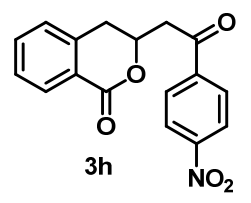
1H), $3.36(\mathrm{dd}, J=17.5,6.9 \mathrm{~Hz}, 1 \mathrm{H}), 3.19$ (dd, $J=16.2,3.3 \mathrm{~Hz}, 1 \mathrm{H}), 3.08(\mathrm{dd}, J=16.1,11.2$ $\mathrm{Hz}, 1 \mathrm{H}) ;{ }^{13} \mathbf{C}$ NMR (101 MHz, $\left.\mathbf{C D C l}_{3}\right): \delta, 194.8,164.8,150.7,140.7,138.4,134.1,130.4$, 129.2(2C), 128.0, 127.6, 124.8, 124.1(2C), 74.3, 43.9, 33.1; HR-MS (ESI, m/z): Calculated for $\mathrm{C}_{17} \mathrm{H}_{13} \mathrm{NNaO}_{5}\left([\mathrm{M}+\mathrm{Na}]^{+}\right)$: 334.0686; Found: 334.0673; $[\alpha]_{\mathrm{D}}^{22}=-7.04\left(\mathrm{c}=0.5, \mathrm{CHCl}_{3}\right.$, $93 \%$ ee).

The enantiomeric ratio was determined by HPLC analysis using Diacel Chiralpak OD-3 column, $\mathrm{n}$-Hexane/2-Propanol $=65 / 35$, flow rate $=1.0 \mathrm{~mL} / \mathrm{min}, \lambda=254 \mathrm{~nm} ; \mathrm{t}_{\mathrm{R}}=32.8 \mathrm{~min}$ (major), $\mathrm{t}_{\mathrm{R}}=55.1 \mathrm{~min}$ (minor).

3-(2-(Furan-2-yl)-2-oxoethyl)isochroman-1-one (3i): (before wash $23 \mathrm{mg}$; 65\% yield; 92\% ee); $18 \mathrm{mg} ; 51 \%$ yield; $\mathrm{R}_{\mathrm{f}}=0.18$ (20:80 = EtOAc/n-Hexane); Brown Solid; mp $106{ }^{0} \mathrm{C}$; FTIR (neat): 3090, 3024, 2967, 2852, 1719, 1677, 1456, 1252, 1125, 768 $\mathrm{cm}^{-1} ;{ }^{1} \mathbf{H}$ NMR (400 MHz, $\left.\mathbf{C D C l}_{3}\right): \delta, 8.08(\mathrm{~d}, J=7.7 \mathrm{~Hz}, 1 \mathrm{H}), 7.61(\mathrm{~d}$, $J=0.9 \mathrm{~Hz}, 1 \mathrm{H}), 7.57-7.47$ (m, 1H), 7.40 (d, $J=7.6 \mathrm{~Hz}, 1 \mathrm{H}), 7.24$ (dd, $J$

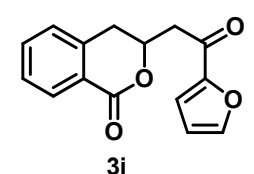
$=5.7,5.0 \mathrm{~Hz}, 2 \mathrm{H}), 6.56(\mathrm{dd}, J=3.6,1.6 \mathrm{~Hz}, 1 \mathrm{H}), 5.15(\mathrm{dddd}, J=10.9,7.4,5.7,3.6 \mathrm{~Hz}, 1 \mathrm{H})$, $3.55(\mathrm{dd}, J=16.6,5.6 \mathrm{~Hz}, 1 \mathrm{H}), 3.18(\mathrm{dd}, J=15.0,5.7 \mathrm{~Hz}, 1 \mathrm{H}), 3.16-2.99(\mathrm{~m}, 2 \mathrm{H}) ;{ }^{13} \mathbf{C}$ NMR (101 MHz, $\left.\mathbf{C D C l}_{3}\right): \delta, 184.9,165.0,152.4,147.0,138.6,133.9,130.3,127.8,127.5$, 124.9, 118.1, 112.6, 74.4, 43.2, 33.1; HR-MS (ESI, $\boldsymbol{m} / \mathbf{z})$ : Calculated for $\mathrm{C}_{15} \mathrm{H}_{13} \mathrm{O}_{4}\left([\mathrm{M}+\mathrm{H}]^{+}\right)$: 257.0808; Found: 257.0813; $[\alpha]_{\mathrm{D}}^{21}=-54.16\left(\mathrm{c}=0.5, \mathrm{CHCl}_{3}, 96 \%\right.$ ee $)$. 
The enantiomeric ratio was determined by HPLC analysis using Diacel Chiralpak OD-3 column, $\mathrm{n}$-Hexane/2-Propanol $=75 / 25$, flow rate $=1.0 \mathrm{~mL} / \mathrm{min}, \lambda=254 \mathrm{~nm} ; \mathrm{t}_{\mathrm{R}}=12.6 \mathrm{~min}$ (major), $\mathrm{t}_{\mathrm{R}}=15.3 \mathrm{~min}$ (minor).

3-(2-Oxo-2-(thiophen-2-yl)ethyl)isochroman-1-one (3j): (before wash $22 \mathrm{mg}$, 83\% yield); $\mathrm{R}_{\mathrm{f}}=0.21$ (30:70 = EtOAc/n-Hexane); Grey sticky liquid; FT-IR (neat): 3095, 3124, 2977, 2844, 1733, 1670, 1455, 967, $751 \mathrm{~cm}^{-1}$; ${ }^{1} \mathbf{H}$ NMR (400 MHz, CDCl 3 ): $\delta$, $8.07(\mathrm{~d}, J=7.6 \mathrm{~Hz}, 1 \mathrm{H}), 7.75(\mathrm{dd}, J=3.8,0.8 \mathrm{~Hz}, 1 \mathrm{H}), 7.68(\mathrm{dd}, J=4.9$, $0.8 \mathrm{~Hz}, 1 \mathrm{H}), 7.53(\mathrm{td}, J=7.5,1.1 \mathrm{~Hz}, 1 \mathrm{H}), 7.38(\mathrm{t}, J=7.6 \mathrm{~Hz}, 1 \mathrm{H}), 7.24$ $(\mathrm{d}, J=6.7 \mathrm{~Hz}, 1 \mathrm{H}), 7.14(\mathrm{dd}, J=4.8,4.0 \mathrm{~Hz}, 1 \mathrm{H}), 5.15(\mathrm{tdd}, J=11.1$,

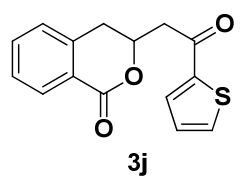
5.3, $3.4 \mathrm{~Hz}, 1 \mathrm{H}), 3.61(\mathrm{dd}, J=16.6,5.3 \mathrm{~Hz}, 1 \mathrm{H}), 3.27(\mathrm{dd}, J=16.6,7.6 \mathrm{~Hz}, 1 \mathrm{H}), 3.18$ (dd, $J$ $=16.2,3.3 \mathrm{~Hz}, 1 \mathrm{H}), 3.05(\mathrm{dd}, J=16.2,11.2 \mathrm{~Hz}, 1 \mathrm{H}) ;{ }^{13} \mathbf{C}$ NMR (101 MHz, CDCl $\left.\mathbf{3}\right): \delta$, $188.9,165.0,143.8,138.6,134.7,133.9,132.7,130.3,128.4,127.9,127.6,124.9,74.6,44.1$, 33.1; HR-MS (ESI, m/z): Calculated for $\mathrm{C}_{15} \mathrm{H}_{13} \mathrm{O}_{3} \mathrm{~S}\left([\mathrm{M}+\mathrm{H}]^{+}\right)$: 273.0580; Found: 273.0594; $[\alpha]_{\mathrm{D}}^{24}=-68.089\left(\mathrm{c}=0.920, \mathrm{CHCl}_{3}, 95 \%\right.$ ee $)$.

The enantiomeric ratio was determined by HPLC analysis using Diacel Chiralpak OD-3 column, $n$-Hexane/2-Propanol $=80 / 20$, flow rate $=1.0 \mathrm{~mL} / \mathrm{min}, \lambda=254 \mathrm{~nm} ; \mathrm{t}_{\mathrm{R}}=17.5 \mathrm{~min}$ (major), $\mathrm{t}_{\mathrm{R}}=20.8 \mathrm{~min}$ (minor).

7-Methyl-3-(2-oxo-2-(p-tolyl)ethyl)isochroman-1-one (3k): $17 \mathrm{mg} ; 53 \%$ yield; $\mathrm{R}_{\mathrm{f}}=0.29$ (20:80 = EtOAc/n-Hexane); White Solid; mp $120{ }^{\circ} \mathrm{C}$; FT-IR (neat): 3019, 2967, 2857, 2369 , 1708, 1605, 1493, 1191, $761 \mathrm{~cm}^{-1}$; ${ }^{1} \mathbf{H}$ NMR (400 MHz, $\left.\mathbf{C D C l}_{3}\right): \delta$, $7.90(\mathrm{~s}, 1 \mathrm{H}), 7.85(\mathrm{~d}, J=8.2 \mathrm{~Hz}, 2 \mathrm{H}), 7.40-7.31(\mathrm{~m}, 1 \mathrm{H}), 7.27$ (d, $J=$ $8.0 \mathrm{~Hz}, 2 \mathrm{H}), 7.13(\mathrm{~d}, J=7.7 \mathrm{~Hz}, 1 \mathrm{H}), 5.24-5.09$ (m, 1H), $3.66(\mathrm{dd}, J$ $=17.2,4.8 \mathrm{~Hz}, 1 \mathrm{H}), 3.31(\mathrm{dd}, J=17.2,8.1 \mathrm{~Hz}, 1 \mathrm{H}), 3.17(\mathrm{dd}, J=16.2$,<smiles>Cc1ccc(C(=O)CC2Cc3ccc(C)cc3C(=O)O2)cc1</smiles>
$3.1 \mathrm{~Hz}, 1 \mathrm{H}), 2.96(\mathrm{dd}, J=16.0,11.2 \mathrm{~Hz}, 1 \mathrm{H}), 2.41(\mathrm{~s}, 3 \mathrm{H}), 2.38(\mathrm{~s}, 3 \mathrm{H}) ;{ }^{13} \mathbf{C}$ NMR (101 MHz, $\left.\mathbf{C D C l}_{3}\right): \delta, 195.9,165.5,144.7,137.7,135.8,134.8,134.0,130.5,129.5$ (2C), 128.2 (2C), 127.5, 124.7, 75.0, 43.3, 32.8, 21.7, 21.0; HR-MS (ESI, m/z): Calculated for $\mathrm{C}_{19} \mathrm{H}_{18} \mathrm{NaO}_{3}\left([\mathrm{M}+\mathrm{Na}]^{+}\right): 317.1148$; Found: $317.1150 ;[\alpha]_{\mathrm{D}}{ }^{22}=-63.78\left(\mathrm{c}=0.5, \mathrm{CHCl}_{3}, 94 \%\right.$ ee).

The enantiomeric ratio was determined by HPLC analysis using Diacel Chiralpak OZ-3 column, $\mathrm{n}$-Hexane/2-Propanol $=60 / 40$, flow rate $=1.0 \mathrm{~mL} / \mathrm{min}, \lambda=254 \mathrm{~nm} ; \mathrm{t}_{\mathrm{R}}=47.2 \mathrm{~min}$ (major), $\mathrm{t}_{\mathrm{R}}=26.4 \mathrm{~min}$ (minor). 
7-Bromo-3-(2-(4-fluorophenyl)-2-oxoethyl)isochroman-1-one (3l): $12 \mathrm{mg}$; 24\% yield; $\mathrm{R}_{\mathrm{f}}=$ 0.22 (20:80 = EtOAc/n-Hexane); White Solid; mp $137{ }^{\circ} \mathrm{C}$; FT-IR (neat): 3041, 2993, 2896, 2830, 2356, 1701, 1686, 1592, 1452, 1221, 1134, $910 \mathrm{~cm}^{-1} ;{ }^{1} \mathbf{H}$ NMR (400 MHz, $\left.\mathbf{C D C l}_{3}\right): \delta, 8.06-7.90(\mathrm{~m}, 3 \mathrm{H}), 7.54(\mathrm{dd}, J=8.3,1.2 \mathrm{~Hz}$, $1 \mathrm{H}), 7.44(\mathrm{~s}, 1 \mathrm{H}), 7.15$ (t, $J=8.6 \mathrm{~Hz}, 2 \mathrm{H}), 5.17$ (tdd, $J=8.0,4.9,3.3$ $\mathrm{Hz}, 1 \mathrm{H}), 3.66(\mathrm{dd}, J=17.4,5.0 \mathrm{~Hz}, 1 \mathrm{H}), 3.30(\mathrm{dd}, J=17.4,7.7 \mathrm{~Hz}$,

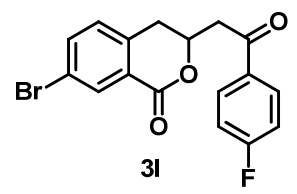
1H), $3.18(\mathrm{dd}, J=16.3,3.2 \mathrm{~Hz}, 1 \mathrm{H}), 3.02(\mathrm{dd}, J=16.3,11.2 \mathrm{~Hz}, 1 \mathrm{H}) ;{ }^{13} \mathbf{C}$ NMR (101 MHz, $\left.\mathbf{C D C l}_{3}\right): \delta, 194.3,166.1(\mathrm{~d}, J=256.2 \mathrm{~Hz}), 164.3,140.4,132.8(\mathrm{~d}, J=3.0 \mathrm{~Hz}), 131.9,131.4$, 130.9, 130.8, 130.7, 129.1, 123.8, 116.1, 115.9, 74.6, 43.2, 32.9; HR-MS (ESI, m/z): Calculated for $\mathrm{C}_{17} \mathrm{H}_{13} \mathrm{BrFO}_{3}\left([\mathrm{M}+\mathrm{H}]^{+}\right)$: 363.0027; Found: $363.0025 ;[\alpha]_{\mathrm{D}}{ }^{17}=-43.08(\mathrm{c}=0.5$, $\mathrm{CHCl}_{3},>99 \%$ ee).

The enantiomeric ratio was determined by HPLC analysis using Diacel Chiralpak OD-3 column, $\mathrm{n}$-Hexane/2-Propanol $=75 / 25$, flow rate $=1.0 \mathrm{~mL} / \mathrm{min}, \lambda=254 \mathrm{~nm} ; \mathrm{t}_{\mathrm{R}}=17.9 \mathrm{~min}$ (major), $\mathrm{t}_{\mathrm{R}}=27.5 \mathrm{~min}$ (minor).

Representative synthetic procedure for synthesis of substrates 4a-f, see: ref. 2

For experimental data for $4 a$ and $4 c$, see: ref. 2

(E)-2-(2-(3-(4-Bromophenyl)-3-oxoprop-1-en-1-yl)-4-methylphenyl)acetaldehyde (4b): $85 \mathrm{mg} ; 46 \%$ yield; $\mathrm{R}_{\mathrm{f}}=0.15$ (10:90 = EtOAc/n-Hexane); Yellow colour oil; FT-IR (neat): 3019, 2896, 2843, 2334, 1719, 1596, 1484, 1322, 1070, 1029, 823, $698 \mathrm{~cm}^{-1} ;{ }^{1} \mathbf{H}$ NMR (400 MHz, $\left.\mathbf{C D C l}_{3}\right): \delta, 9.72(\mathrm{~s}, 1 \mathrm{H}), 7.90(\mathrm{~d}, J$ $=15.5 \mathrm{~Hz}, 1 \mathrm{H}), 7.87(\mathrm{~d}, J=8.5 \mathrm{~Hz}, 2 \mathrm{H}), 7.62(\mathrm{~d}, J=8.5 \mathrm{~Hz}, 2 \mathrm{H})$,

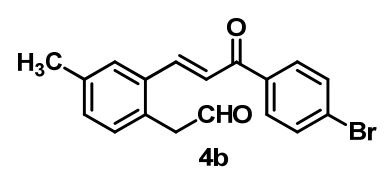
7.56 (s, 1H), 7.39 (d, $J=15.4 \mathrm{~Hz}, 1 \mathrm{H}), 7.21(\mathrm{~d}, J=7.7 \mathrm{~Hz}, 1 \mathrm{H}), 7.11(\mathrm{~d}, J=7.8 \mathrm{~Hz}, 1 \mathrm{H})$, 3.87 (d, $J=1.4 \mathrm{~Hz}, 2 \mathrm{H}), 2.38$ (s, 3H); ${ }^{13} \mathbf{C}$ NMR (101 MHz, $\left.\mathbf{C D C l}_{3}\right): \delta, 198.4,188.9,141.9$, 137.9, 136.7, 134.3, 131.9 (2C), 131.8, 131.5, 130.1 (2C), 129.4, 128.1, 127.7, 123.6, 47.9, 21.1; HR-MS (ESI, $\boldsymbol{m} / \mathbf{z})$ : Calculated for $\mathrm{C}_{18} \mathrm{H}_{15} \mathrm{BrO}_{2}\left([\mathrm{M}+\mathrm{H}]^{+}\right)$: 343.0328; Found: 343.0340.

(E)-2-(2-(3-(4-Bromophenyl)-3-oxoprop-1-en-1-yl)-6-fluorophenyl)acetaldehyde

(4d): $70 \mathrm{mg} ; 67 \%$ yield; $\mathrm{R}_{\mathrm{f}}=0.15$ (20:80 = EtOAc/n-Hexane); Yellow colour oil; FT-IR (neat): 2975, 2835, 2364, 1680, 1467, 1243, 1072, 831, $700 \mathrm{~cm}^{-1}$; ${ }^{1} \mathbf{H}$ NMR (400 MHz, $\left.\mathbf{C D C l}_{3}\right): \delta, 9.76(\mathrm{~d}, J=1.0 \mathrm{~Hz}, 1 \mathrm{H}), 7.84(\mathrm{t}, J=11.4 \mathrm{~Hz}$,

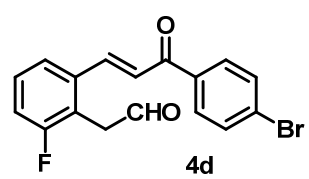


3H), $7.62(\mathrm{~d}, J=8.5 \mathrm{~Hz}, 2 \mathrm{H}), 7.52(\mathrm{~d}, J=7.9 \mathrm{~Hz}, 1 \mathrm{H}), 7.39(\mathrm{~d}, J=15.4 \mathrm{~Hz}, 1 \mathrm{H}), 7.34(\mathrm{td}, J$

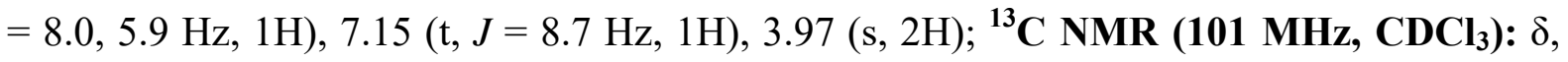
196.9, 188.7, 161.5 (d, $J=246.3 \mathrm{~Hz}), 140.6(\mathrm{~d}, J=3.5 \mathrm{~Hz}), 136.8$ (d, $J=4.0 \mathrm{~Hz}), 136.4$, 132.1 (2C), 130.1 (2C), 129.2 (d, $J=9.1 \mathrm{~Hz}), 128.4,125.1,122.87$ (d, $J=3.2 \mathrm{~Hz}), 119.9$ (d, $J=16.4 \mathrm{~Hz}), 116.96(\mathrm{~d}, J=23.0 \mathrm{~Hz}), 40.4$ (d, $J=3.5 \mathrm{~Hz})$; HR-MS (ESI, m/z): Calculated for $\mathrm{C}_{17} \mathrm{H}_{13} \mathrm{BrFO}_{2}\left([\mathrm{M}+\mathrm{H}]^{+}\right)$: 347.0077 ; Found: 347.0049 .

(E)-2-(2-(3-(4-Bromophenyl)-3-oxoprop-1-en-1-yl)-5-fluorophenyl)acetaldehyde (4e): 73 mg; $58 \%$ yield; $\mathrm{R}_{\mathrm{f}}=0.17(20: 80=$ EtOAc/n-Hexane); Yellow colour sticky liquid; FT-IR (neat): 3017, 2976, 2856, 1678, 1480, 1225, 1070, 975, $768 \mathrm{~cm}^{-1} ;{ }^{1} \mathbf{H}$ NMR (400 MHz, $\left.\mathbf{C D C l}_{3}\right): \delta, 9.76(\mathrm{~s}, 1 \mathrm{H}), 7.85(\mathrm{dd}, J=11.9,3.4 \mathrm{~Hz}, 3 \mathrm{H}), 7.75(\mathrm{dd}, J=$ 8.7, $5.7 \mathrm{~Hz}, 1 \mathrm{H}), 7.63(\mathrm{~d}, J=8.6 \mathrm{~Hz}, 2 \mathrm{H}), 7.35$ (d, $J=15.4 \mathrm{~Hz}, 1 \mathrm{H})$, $7.07(\mathrm{td}, J=8.3,2.6 \mathrm{~Hz}, 1 \mathrm{H}), 6.96(\mathrm{dd}, J=9.0,2.6 \mathrm{~Hz}, 1 \mathrm{H}), 3.92(\mathrm{~d}, J$

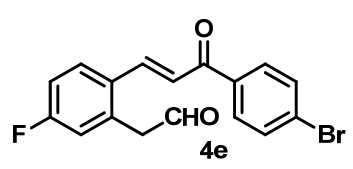
$=1.2 \mathrm{~Hz}, 2 \mathrm{H}) ;{ }^{13} \mathbf{C} \mathbf{N M R}\left(\mathbf{1 0 1} \mathbf{M H z}, \mathbf{C D C l}_{\mathbf{3}}\right): \delta, 197.2,188.7,163.8(\mathrm{~d}, J=252.7 \mathrm{~Hz}), 140.5$, 136.5, 134.9 (d, $J=8.1 \mathrm{~Hz}), 132.0$ (2C), 130.8 (d, $J=3.4 \mathrm{~Hz}), 130.1$ (2C), 129.2 (d, $J=8.8$ Hz), 128.2, 123.6 (d, $J=2.0 \mathrm{~Hz}), 118.4$ (d, $J=21.9 \mathrm{~Hz}), 115.5$ (d, $J=21.6 \mathrm{~Hz}), 48.0$ (d, $J=$ $1.3 \mathrm{~Hz}$ ); HR-MS (ESI, m/z): Calculated for $\mathrm{C}_{17} \mathrm{H}_{12} \mathrm{BrFNaO}_{2}\left([\mathrm{M}+\mathrm{Na}]^{+}\right)$: 368.9897; Found: 368.9893 .

\section{Representative synthetic procedure for 3-Isochromanones:}

\section{Step-I}

A solution of chalcone 4 ( 1 equiv, $0.1 \mathrm{mmol}$ ) and catalyst (5 mol\%) in toluene was taken in a small round bottom flask with a magnetic bar and was placed over a magnetic stirrer at room temperature. Then $6 \mathrm{M}$ solution of TBHP (5 equiv) was added dropwise to the pre-mix solution. Then the reaction mixture was allowed to stir at room temperature for $12-15 \mathrm{~h}$. The reaction was stopped when all the starting material (chalcone) were consumed (monitored by TLC). Finally, the reaction was filtered with a small plug of silica and washed with dichloromethane as solvent. The peroxy-acetal was directly taken for step-II after removing the solvent with vaccuo.

\section{Step-II}


The solution of peroxy-acetal (obtained from step-I) in dichloromethane was taken in a small round bottom flask with a bar magnet and was placed over a magnetic stirrer. Solid $\mathrm{FeCl}_{3} \cdot 6 \mathrm{H}_{2} \mathrm{O}(25 \mathrm{~mol} \%)$ was directly added to the flask and the reaction was allowed to stir at room temperature for overnight. Then, the reaction mixture was filtered through a small plug of silica gel and washed with dichloromethane as solvent. After the removal of solvent in vaccuo, the crude 3-Isochromanone was washed with n-hexane to get optically pure 3Isochromanone.

1-(2-(4-Bromophenyl)-2-oxoethyl)isochroman-3-one (5a): (before wash $49 \mathrm{mg}$; 95\% yield; $83 \%$ ee); 24 mg; 46\% yield; $\mathrm{R}_{\mathrm{f}}=0.27$ (20:80 = EtOAc/n-Hexane); Brown Solid; mp $143{ }^{0} \mathrm{C}$; FT-IR (neat): 3020, 2892, 2836, 2358, 1662, 1535, 1022, $691 \mathrm{~cm}^{-1}$; ${ }^{1}$ H NMR (400 MHz, CDCl $)$ ): $\delta, 7.84(\mathrm{~d}, J=3.9 \mathrm{~Hz}, 2 \mathrm{H}), 7.63(\mathrm{dd}, J$ $=5.3,2.8 \mathrm{~Hz}, 2 \mathrm{H}), 7.32(\mathrm{dd}, J=12.0,8.1 \mathrm{~Hz}, 2 \mathrm{H}), 7.23(\mathrm{dd}, J=10.8$, $7.5 \mathrm{~Hz}, 2 \mathrm{H}), 6.08(\mathrm{~d}, J=4.0 \mathrm{~Hz}, 1 \mathrm{H}), 3.78(\mathrm{~d}, J=2.2 \mathrm{~Hz}, 2 \mathrm{H}), 3.74(\mathrm{~d}$,

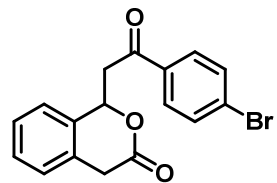

5 a $J=6.1 \mathrm{~Hz}, 1 \mathrm{H}), 3.67-3.53(\mathrm{~m}, 1 \mathrm{H}) ;{ }^{13} \mathbf{C}$ NMR (101 MHz, $\left.\mathbf{C D C l}_{3}\right): \delta, 194.8,170.1,135.1$, 133.8, 132.2 (2C), 130.8, 129.7 (2C), 129.1, 129.0, 127.5, 127.4, 123.9, 76.1, 41.9, 36.2; HRMS (ESI, $\boldsymbol{m} / \mathbf{z})$ : Calculated for $\mathrm{C}_{17} \mathrm{H}_{13} \mathrm{BrNaO}_{3}\left([\mathrm{M}+\mathrm{Na}]^{+}\right): 366.9940$; Found: $366.9953 ;[\alpha]_{\mathrm{D}}{ }^{21}$ $=+18.52\left(\mathrm{c}=0.5, \mathrm{CHCl}_{3}, 92 \%\right.$ ee $)$.

The enantiomeric ratio was determined by HPLC analysis using Diacel Chiralpak OD-3 column, $\mathrm{n}$-Hexane/2-Propanol $=65 / 35$, flow rate $=1.0 \mathrm{~mL} / \mathrm{min}, \lambda=254 \mathrm{~nm} ; \mathrm{t}_{\mathrm{R}}=20.1 \mathrm{~min}$ (major), $\mathrm{t}_{\mathrm{R}}=36.8 \mathrm{~min}$ (minor).

1-(2-(4-Bromophenyl)-2-oxoethyl)-7-methylisochroman-3-one (5b): (before wash $29 \mathrm{mg}$; $75 \%$ yield; 78\% ee); 20 mg; 52\% yield; $\mathrm{R}_{\mathrm{f}}=0.29$ (20:80 = EtOAc/n-Hexane); White Solid; mp $189{ }^{0} \mathrm{C}$; FT-IR (neat): 3073, 2994, 2896, 2829, 1741, 1686, 1492, 1066, 820, $720 \mathrm{~cm}^{-1} ;{ }^{1} \mathbf{H}$ NMR (400 MHz, $\left.\mathbf{C D C l}_{3}\right): \delta, 7.91-7.81(\mathrm{~m}$, 2H), $7.68-7.60(\mathrm{~m}, 2 \mathrm{H}), 7.22-7.07(\mathrm{~m}, 2 \mathrm{H}), 7.03-6.89(\mathrm{~m}, 1 \mathrm{H})$, $6.07(\mathrm{dt}, J=12.5,6.4 \mathrm{~Hz}, 1 \mathrm{H}), 4.04-3.66(\mathrm{~m}, 3 \mathrm{H}), 3.62-3.47(\mathrm{~m}$,

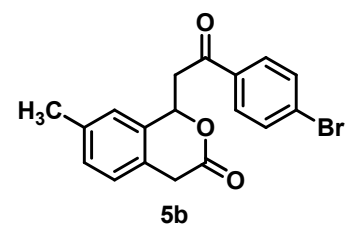
1H), 2.40 - 2.24 (m, 3H); ${ }^{13} \mathbf{C}$ NMR (101 MHz, $\left.\mathbf{C D C l}_{3}\right): \delta, 194.8,170.3,137.4,135.2$, 133.7, 132.2(3C), 129.7 (2C), 129.7, 127.7, 127.3, 124.5, 76.2, 42.1, 35.8, 21.3; HR-MS (ESI, $\boldsymbol{m} / \mathbf{z}$ ): Calculated for $\mathrm{C}_{18} \mathrm{H}_{16} \mathrm{BrO}_{3}\left([\mathrm{M}+\mathrm{H}]^{+}\right)$: 359.0277 ; Found: 359.0294 ; $[\alpha]_{\mathrm{D}}{ }^{20}=+8.86$ (c $=0.5, \mathrm{CHCl}_{3}, 95 \%$ ee). 
The enantiomeric ratio was determined by HPLC analysis using Diacel Chiralpak OD-3 column, $\mathrm{n}$-Hexane/2-Propanol $=65 / 35$, flow rate $=1.0 \mathrm{~mL} / \mathrm{min}, \lambda=254 \mathrm{~nm} ; \mathrm{t}_{\mathrm{R}}=21.5 \mathrm{~min}$ (major), $\mathrm{t}_{\mathrm{R}}=25.5 \mathrm{~min}$ (minor).

1-(2-(4-Bromophenyl)-2-oxoethyl)-6-chloroisochroman-3-one (5c): (before wash $80 \mathrm{mg}$; 82\% yield; 93\% ee); $48 \mathrm{mg} ; 49 \%$ yield; $\mathrm{R}_{\mathrm{f}}=0.28$ (20:80 = EtOAc/n-Hexane); White Solid; mp $153{ }^{0} \mathrm{C}$; FT-IR (neat): 3032, 2940, 2852, 2369, 1747, 1684, 1452, 1239, 1210, 1070, 814, $702 \mathrm{~cm}^{-1} ;{ }^{1} \mathbf{H}$ NMR (400 MHz, $\left.\mathbf{C D C l}_{3}\right): \delta$, $7.82(\mathrm{~d}, J=8.5 \mathrm{~Hz}, 2 \mathrm{H}), 7.62(\mathrm{~d}, J=8.5 \mathrm{~Hz}, 2 \mathrm{H}), 7.25(\mathrm{dd}, J=13.5$,

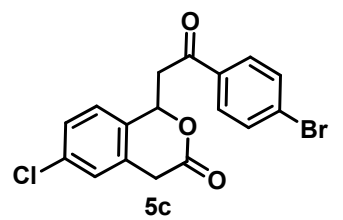
$5.7 \mathrm{~Hz}, 2 \mathrm{H}), 7.13(\mathrm{~d}, J=8.2 \mathrm{~Hz}, 1 \mathrm{H}), 6.03(\mathrm{t}, J=6.2 \mathrm{~Hz}, 1 \mathrm{H}), 3.76(\mathrm{~d}, J=4.8 \mathrm{~Hz}, 2 \mathrm{H}), 3.72$ $(\mathrm{d}, J=6.0 \mathrm{~Hz}, 1 \mathrm{H}), 3.57(\mathrm{dd}, J=17.3,6.5 \mathrm{~Hz}, 1 \mathrm{H}) ;{ }^{13} \mathbf{C} \mathbf{N M R}\left(\mathbf{1 0 1} \mathbf{M H z}, \mathbf{C D C l}_{3}\right) \delta, 194.5$, 169.2, 134.9, 134.8, 132.7, 132.3, 132.2 (2C), 129.7 (2C), 129.3, 127.7, 127.5, 125.5, 75.7, 42.0, 35.9; HR-MS (ESI, m/z): Calculated for $\mathrm{C}_{17} \mathrm{H}_{12} \mathrm{BrClNaO}_{3}\left([\mathrm{M}+\mathrm{Na}]^{+}\right)$: 400.9551; Found: $400.9561 ;[\alpha]_{\mathrm{D}}^{22}=+72.266\left(\mathrm{c}=0.5, \mathrm{CHCl}_{3}, 98 \%\right.$ ee $)$.

The enantiomeric ratio was determined by HPLC analysis using Diacel Chiralpak OD-3 column, $n-H e x a n e / 2-P r o p a n o l=65 / 35$, flow rate $=1.0 \mathrm{~mL} / \mathrm{min}, \lambda=254 \mathrm{~nm} ; \mathrm{t}_{\mathrm{R}}=23.9 \mathrm{~min}$ (major), $\mathrm{t}_{\mathrm{R}}=33.3 \mathrm{~min}$ (minor).

1-(2-(4-Bromophenyl)-2-oxoethyl)-6-fluoroisochroman-3-one (5d): (before wash $43 \mathrm{mg}$; 93\% yield; 88\% ee); $23 \mathrm{mg} ; 50 \%$ yield; $\mathrm{R}_{\mathrm{f}}=0.25$ (20:80 = EtOAc/n-Hexane); Light Yellow Solid; mp $134{ }^{0} \mathrm{C}$; FT-IR (neat): 3029, 2957, 2853, 2360, 1752, 1673, 1500, 1258, $698 \mathrm{~cm}^{-1} ;{ }^{1} \mathbf{H}$ NMR (400 MHz, $\left.\mathbf{C D C l}_{3}\right): \delta, 7.84(\mathrm{t}, J=$ $10.3 \mathrm{~Hz}, 2 \mathrm{H}), 7.63(\mathrm{~d}, J=8.6 \mathrm{~Hz}, 2 \mathrm{H}), 7.17(\mathrm{dd}, J=8.4,5.2 \mathrm{~Hz}, 1 \mathrm{H})$, $7.08-6.83(\mathrm{~m}, 2 \mathrm{H}), 6.12-5.97(\mathrm{~m}, 1 \mathrm{H}), 3.77(\mathrm{~s}, 2 \mathrm{H}), 3.76-3.71(\mathrm{~m}$,

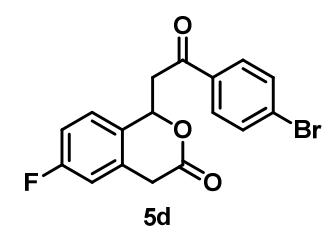
1H), $3.58(\mathrm{dd}, J=17.2,6.5 \mathrm{~Hz}, 1 \mathrm{H}) ;{ }^{13} \mathbf{C}$ NMR (101 MHz, $\left.\mathbf{C D C l}_{3}\right): \delta, 194.6,169.3,162.8$ (d, $J=248.9 \mathrm{~Hz}), 134.9,133.3(\mathrm{~d}, J=8.7 \mathrm{~Hz}), 132.2$ (2C), 129.69 (2C), 129.66, 129.3, 125.9 (d, $J=8.8 \mathrm{~Hz}), 114.6(\mathrm{~d}, J=5.5 \mathrm{~Hz}), 114.43(\mathrm{~d}, J=4.6 \mathrm{~Hz}), 75.7,42.1,36.17(\mathrm{~d}, J=1.6$ $\mathrm{Hz}$ ); HR-MS (ESI, $\boldsymbol{m} / \mathbf{z})$ : Calculated for $\mathrm{C}_{17} \mathrm{H}_{12} \mathrm{BrNaFO}_{3}\left([\mathrm{M}+\mathrm{Na}]^{+}\right)$: 384.9846; Found: $384.9828 ;[\alpha]_{\mathrm{D}}^{21}=+33.6\left(\mathrm{c}=0.5, \mathrm{CHCl}_{3}, 98 \%\right.$ ee $)$.

The enantiomeric ratio was determined by HPLC analysis using Diacel Chiralpak OD-3 column, $\mathrm{n}$-Hexane/2-Propanol $=65 / 35$, flow rate $=1.0 \mathrm{~mL} / \mathrm{min}, \lambda=254 \mathrm{~nm} ; \mathrm{t}_{\mathrm{R}}=19.8 \mathrm{~min}$ (major), $\mathrm{t}_{\mathrm{R}}=51.6 \mathrm{~min}$ (minor).

Representative synthetic procedure for synthesis of substrates 6a-y, see: ref. 1 
Experimental data for 6a-6d, 6f, 6i-k, 6m-p, 6r-t, 6v, see: ref. 1

(E)-2-(3-(2-Bromophenyl)-3-oxoprop-1-en-1-yl)benzaldehyde (6f): $209 \mathrm{mg} ; 70 \%$ yield; $\mathrm{R}_{\mathrm{f}}$ $=0.25(10: 90=\mathrm{EtOAc} / \mathrm{n}-\mathrm{Hexane})$; Brown Solid; $\mathrm{mp} 76{ }^{0} \mathrm{C}$; FT-IR (neat): 3024, 2854, 2364, 1699, 1653, 1603, 1494, 1454, 1292, $1031 \mathrm{~cm}^{-1}$; ${ }^{1} \mathbf{H}$ NMR (400 MHz, CDCl 3 ): $\delta, 10.20$ (s, $1 \mathrm{H}), 8.27(\mathrm{~d}, J=16.1 \mathrm{~Hz}, 1 \mathrm{H}), 7.85$ (dd, $J=7.5,1.1 \mathrm{~Hz}, 1 \mathrm{H}), 7.72$ (d, $J=7.6 \mathrm{~Hz}, 1 \mathrm{H}), 7.63(\mathrm{dd}, J=12.0,4.5 \mathrm{~Hz}, 2 \mathrm{H}), 7.58(\mathrm{dt}, J=7.4,3.8$ $\mathrm{Hz}, 1 \mathrm{H}), 7.48(\mathrm{dd}, J=7.5,1.7 \mathrm{~Hz}, 1 \mathrm{H}), 7.46-7.40$ (m, 1H), 7.34 (td,

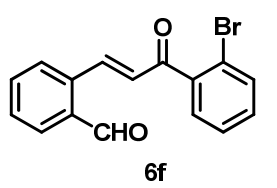
$J=7.8,1.8 \mathrm{~Hz}, 1 \mathrm{H}), 6.98(\mathrm{~d}, J=16.1 \mathrm{~Hz}, 1 \mathrm{H}) ;{ }^{13} \mathbf{C}$ NMR (101 MHz, CDCl $\left.\mathbf{3}\right): \delta, 194.7$, $191.9,143.5,140.6,136.4,134.0,133.9,133.5,132.9,131.6,130.5,130.4,129.4,128.0$, 127.4, 119.6; HR-MS (ESI, m/z): Calculated for $\mathrm{C}_{16} \mathrm{H}_{12} \mathrm{BrO}_{2}\left([\mathrm{M}+\mathrm{H}]^{+}\right)$: 315.0015; Found: 315.0030 .

(E)-2-(3-(4-Iodophenyl)-3-oxoprop-1-en-1-yl)benzaldehyde (6g): $220 \mathrm{mg}$; 65\% yield; $\mathrm{R}_{\mathrm{f}}=$ 0.20 (20:80 = EtOAc/n-Hexane); Yellow Solid; mp $119{ }^{0}$ C; FT-IR (neat): 3015, 2984, 2870, 2839, 2364, 1605, 1585, 1452, 1285, 1032, $755 \mathrm{~cm}^{-1} ;{ }^{1} \mathbf{H}$ NMR (400 MHz, CDCl$): ~ \delta 10.30$ (s, 1H), $8.55(\mathrm{~d}, J=15.7 \mathrm{~Hz}, 1 \mathrm{H}), 7.89$ (dd, $J=12.7,4.9 \mathrm{~Hz}, 3 \mathrm{H})$, $7.73(\mathrm{dd}, J=7.4,5.5 \mathrm{~Hz}, 3 \mathrm{H}), 7.65$ (td, $J=7.5,1.3 \mathrm{~Hz}, 1 \mathrm{H}), 7.62$ - $7.53(\mathrm{~m}, 1 \mathrm{H}), 7.26$ (d, $J=15.7 \mathrm{~Hz}, 1 \mathrm{H}) ;{ }^{13} \mathbf{C}$ NMR (101 MHz,

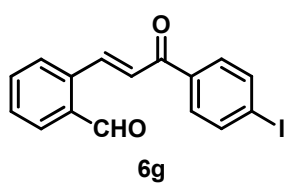
$\left.\mathbf{C D C l}_{3}\right): \delta 191.9,190.0,142.1,138.0(2 \mathrm{C}), 136.99,136.95,134.3,133.9,132.7,130.2,130.1$ (2C), 128.1, 126.8, 100.9; HR-MS (ESI, m/z): Calculated for $\mathrm{C}_{16} \mathrm{H}_{12} \mathrm{IO}_{2}\left([\mathrm{M}+\mathrm{H}]^{+}\right)$: 362.9876; Found: 362.9896.

(E)-2-(3-(3-Nitrophenyl)-3-oxoprop-1-en-1-yl)benzaldehyde (6k): $300 \mathrm{mg} ; 87 \%$ yield; $\mathrm{R}_{\mathrm{f}}$ $=0.14\left(20: 80=\right.$ EtOAc/n-Hexane); White Solid; mp $137{ }^{0} \mathrm{C}$; FT-IR (neat): 3015, 2993, 2887, 2843, 2329, 1673, 1594, 1535, 1351, 1270, 1210, 1086, $693 \mathrm{~cm}^{-1}$; ${ }^{1} \mathbf{H}$ NMR (400 MHz, $\left.\mathbf{C D C l}_{3}\right): \delta, 10.26(\mathrm{~s}, 1 \mathrm{H}), 8.86-8.75(\mathrm{~m}, 1 \mathrm{H}), 8.63(\mathrm{~d}, J=15.8$ $\mathrm{Hz}, 1 \mathrm{H}), 8.42(\mathrm{dd}, J=8.2,1.1 \mathrm{~Hz}, 1 \mathrm{H}), 8.35(\mathrm{~d}, J=7.8 \mathrm{~Hz}, 1 \mathrm{H})$, $7.89(\mathrm{dd}, J=7.4,1.3 \mathrm{~Hz}, 1 \mathrm{H}), 7.77$ (d, $J=7.5 \mathrm{~Hz}, 1 \mathrm{H}), 7.72(\mathrm{t}, J=$

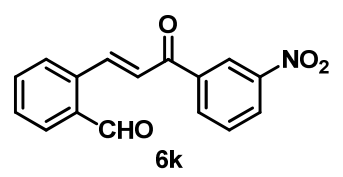
$8.0 \mathrm{~Hz}, 1 \mathrm{H}), 7.66(\mathrm{dd}, J=7.4,1.5 \mathrm{~Hz}, 1 \mathrm{H}), 7.62(\mathrm{dd}, J=7.3,6.3 \mathrm{~Hz}, 1 \mathrm{H}), 7.33$ (d, $J=15.7$ $\mathrm{Hz}, 1 \mathrm{H}) ;{ }^{13} \mathbf{C}$ NMR (101 MHz, $\left.\mathbf{C D C l}_{3}\right): \delta, 192.2,188.7,148.4,143.8,139.0,136.3,134.5$, 134.3, 134.1, 133.4, 130.6, 129.9, 128.2, 127.2, 125.9, 123.6; HR-MS (ESI, m/z): Calculated for $\mathrm{C}_{16} \mathrm{H}_{12} \mathrm{NO}_{4}\left([\mathrm{M}+\mathrm{H}]^{+}\right): 282.0761$; Found: 282.0772 .

(E)-5-Methoxy-2-(3-oxo-3-(p-tolyl)prop-1-en-1-yl)benzaldehyde (6o): $205 \mathrm{mg}$; 80\% yield; $\mathrm{R}_{\mathrm{f}}=0.25$ (20:80 = EtOAc/n-Hexane); Yellow Solid; mp $104{ }^{0} \mathrm{C}$; FT-IR (neat): 2914, 2830,

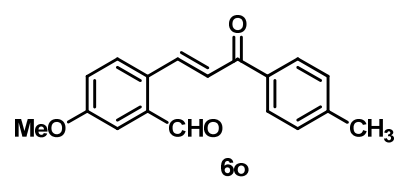


2364, 1682, 1660, 1598, 1452, 1294, 1259, 1031, 814, $755 \mathrm{~cm}^{-1} ;{ }^{1}$ H NMR (400 MHz, $\left.\mathbf{C D C l}_{3}\right): \delta, 10.35(\mathrm{~d}, J=2.1 \mathrm{~Hz}, 1 \mathrm{H}), 8.46(\mathrm{~d}, J=15.5 \mathrm{~Hz}, 1 \mathrm{H}), 7.89$ (d, $\left.J=7.3 \mathrm{~Hz}, 2 \mathrm{H}\right), 7.69$ $(\mathrm{d}, J=8.5 \mathrm{~Hz}, 1 \mathrm{H}), 7.38-7.29(\mathrm{~m}, 2 \mathrm{H}), 7.26(\mathrm{~d}, J=7.4 \mathrm{~Hz}, 2 \mathrm{H}), 7.13(\mathrm{~d}, J=8.6 \mathrm{~Hz}, 1 \mathrm{H})$, $3.86(\mathrm{~d}, J=1.3 \mathrm{~Hz}, 3 \mathrm{H}), 2.39$ (s, 3H); ${ }^{13} \mathbf{C}$ NMR (101 MHz, $\mathbf{C D C l}_{3}$ ): $\delta$ 190.9, 189.8, 161.1, $143.8,139.5,135.5,135.3,130.0,129.5,129.4$ (2C), 128.8 (2C), 125.3, 120.8, 114.3, 55.7, 21.7; HR-MS (ESI, $\mathbf{m} / \mathbf{z})$ : Calculated for $\mathrm{C}_{18} \mathrm{H}_{17} \mathrm{O}_{3}\left([\mathrm{M}+\mathrm{H}]^{+}\right)$: 281.1172; Found: 281.1186.

(E)-5-Bromo-2-(3-(4-bromophenyl)-3-oxoprop-1-en-1-yl)benzaldehyde (6s): $235 \mathrm{mg}$; $68 \%$ yield; $\mathrm{R}_{\mathrm{f}}=0.28$ (10:90 = EtOAc/n-Hexane); White Solid; mp $125{ }^{0} \mathrm{C}$; FT-IR (neat): 3076, 2984, 2883, 2839, 2364, 1662, 1605, 1585, 1458, 1276, 1116, 1068, $702 \mathrm{~cm}^{-1} ;{ }^{1} \mathbf{H}$

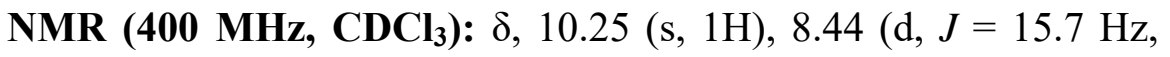
$1 \mathrm{H}), 8.01(\mathrm{~d}, J=1.9 \mathrm{~Hz}, 1 \mathrm{H}), 7.87(\mathrm{~d}, J=8.5 \mathrm{~Hz}, 2 \mathrm{H}), 7.76(\mathrm{dd}, J=$ $8.3,1.8 \mathrm{~Hz}, 1 \mathrm{H}), 7.64(\mathrm{~d}, J=8.5 \mathrm{~Hz}, 2 \mathrm{H}), 7.60(\mathrm{~d}, J=8.3 \mathrm{~Hz}, 1 \mathrm{H})$,

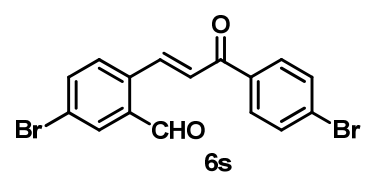
$7.30(\mathrm{~d}, J=15.7 \mathrm{~Hz}, 1 \mathrm{H}) ;{ }^{13} \mathbf{C}$ NMR (101 MHz, $\left.\mathbf{C D C l}_{3}\right): \delta, 190.1,189.2,140.4,136.9$, 136.2, 135.7, 135.4, 134.9, 132.1 (2C), 130.3 (2C), 129.6, 128.4, 127.1, 124.6; HR-MS (ESI, $\boldsymbol{m} / \mathbf{z})$ : Calculated for $\mathrm{C}_{16} \mathrm{H}_{9} \mathrm{Br}_{2} \mathrm{O}_{2}\left([\mathrm{M}-\mathrm{H}]^{+}\right)$: 390.8964; Found: 390.9040.

(E)-2-(3-(4-Bromophenyl)-3-oxoprop-1-en-1-yl)-6-fluorobenzaldehyde (6u): 200 mg; $70 \%$ yield; $\mathrm{R}_{\mathrm{f}}=0.22(10: 90=$ EtOAc/n-Hexane $)$; White colour solid; mp $170{ }^{\circ} \mathrm{C}$; FT-IR (neat): 3070, 2978, 2870, 2840, 2366, 1662, 1605, 1585, 1459, 1276, 1126, 1068, $710 \mathrm{~cm}^{-1} ;{ }^{\mathbf{1}} \mathbf{H}$ NMR (400 MHz, $\left.\mathbf{C D C l}_{3}\right): \delta$, $10.31(\mathrm{~d}, J=1.5 \mathrm{~Hz}, 1 \mathrm{H}), 8.46(\mathrm{~d}, J=15.6 \mathrm{~Hz}, 1 \mathrm{H}), 7.96(\mathrm{~d}, J=$

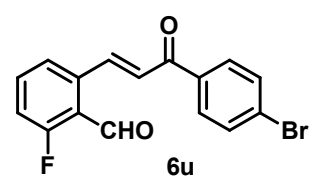
$8.6 \mathrm{~Hz}, 2 \mathrm{H}), 7.74(\mathrm{dd}, J=8.6,5.1 \mathrm{~Hz}, 1 \mathrm{H}), 7.60$ (dd, $J=8.4,2.7 \mathrm{~Hz}, 1 \mathrm{H}), 7.48$ (d, $J=8.6 \mathrm{~Hz}$, 2H), 7.35 (td, $J=8.2,2.7 \mathrm{~Hz}, 1 \mathrm{H}), 7.29$ (d, $J=15.6 \mathrm{~Hz}, 1 \mathrm{H}) ;{ }^{13} \mathbf{C}$ NMR (101 MHz, CDCl $\left.\mathbf{3}\right)$ : $\delta, 189.9,188.9,164.9,162.4,140.2,139.7,136.1,136.0,135.9,133.3,130.4,130.3,130.1$, 129.1, 126.7, 121.4, 121.2, 118.1, 117.8; HR-MS (ESI, m/z): Calculated for $\mathrm{C}_{16} \mathrm{H}_{9} \mathrm{BrFO}_{2}$ $\left([\mathrm{M}-\mathrm{H}]^{+}\right): 330.9764$; Found: 330.9779.

(E)-2-Fluoro-6-(3-oxo-3-(p-tolyl)prop-1-en-1-yl)benzaldehyde (6v): $102 \mathrm{mg}$; 69\% yield; $\mathrm{R}_{\mathrm{f}}=0.18\left(10: 90=\right.$ EtOAc/n-Hexane); Yellow Solid; mp $108{ }^{0} \mathrm{C}$; FT-IR (neat): 3015, 2905, 2835, 2356, 1664, 1601, 1469, 1237, 1180, 1033, 827, $796 \mathrm{~cm}^{-1}$; ${ }^{1} \mathbf{H}$ NMR (400 MHz, $\left.\mathbf{C D C l}_{3}\right): \delta, 10.54$

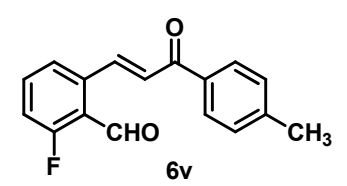
(s, 1H), 8.37 (d, $J=15.8 \mathrm{~Hz}, 1 \mathrm{H}), 7.92(\mathrm{~d}, J=8.2 \mathrm{~Hz}, 2 \mathrm{H}), 7.59$ (td, $J=8.0,5.7 \mathrm{~Hz}, 1 \mathrm{H})$, $7.47(\mathrm{~d}, J=7.8 \mathrm{~Hz}, 1 \mathrm{H}), 7.29$ (d, $J=8.0 \mathrm{~Hz}, 2 \mathrm{H}), 7.27-7.23(\mathrm{~m}, 1 \mathrm{H}), 7.21(\mathrm{dd}, J=7.6,6.7$ $\mathrm{Hz}, 1 \mathrm{H}), 2.41$ (s, 3H); ${ }^{13} \mathbf{C}$ NMR (101 MHz, $\left.\mathbf{C D C l}_{3}\right) \delta, 190.5,188.3$ (d, J=10.5 Hz), 165.7 
$(\mathrm{d}, J=258.5 \mathrm{~Hz}), 141.8(\mathrm{~d}, J=2.8 \mathrm{~Hz}), 138.8,135.4(\mathrm{~d}, J=10.3 \mathrm{~Hz}), 135.0,129.4(2 \mathrm{C})$, 129.0 (2C), 127.8, 124.1, 124.07, 122.4 (d, $J=6.6 \mathrm{~Hz}) .117 .3$ (d, $J=21.8 \mathrm{~Hz}), 21.7$; HR-MS (ESI, $\boldsymbol{m} / \mathbf{z}$ ): Calculated for $\mathrm{C}_{17} \mathrm{H}_{14} \mathrm{FO}_{2}\left([\mathrm{M}+\mathrm{H}]^{+}\right)$: 269.0972; Found: 269.0996.

(E)-1-(3-(4-Bromophenyl)-3-oxoprop-1-en-1-yl)-2-naphthaldehyde (6w): $250 \mathrm{mg}$; 68\% yield; $\mathrm{R}_{\mathrm{f}}=0.35\left(10: 90=\right.$ EtOAc/n-Hexane); White Solid; mp $188{ }^{0} \mathrm{C}$; FT-IR (neat): 2940, 2852, 2364, 1664, 1603, 1493, 1390, 1263, 1070, 915, $761 \mathrm{~cm}^{-1}$;

${ }^{1}$ H NMR (400 MHz, $\left.\mathbf{C D C l}_{3}\right): \delta, 10.41(\mathrm{~s}, 1 \mathrm{H}), 8.60(\mathrm{~d}, J=15.6$ $\mathrm{Hz}, 1 \mathrm{H}), 8.17$ (d, $J=8.4 \mathrm{~Hz}, 1 \mathrm{H}), 8.03(\mathrm{~d}, J=8.6 \mathrm{~Hz}, 1 \mathrm{H}), 7.95$ (d,

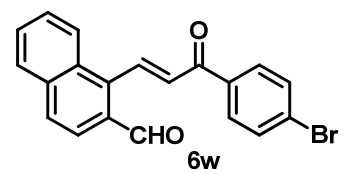
$J=8.8 \mathrm{~Hz}, 2 \mathrm{H}), 7.90(\mathrm{~d}, J=8.6 \mathrm{~Hz}, 2 \mathrm{H}), 7.65(\mathrm{dtd}, J=12.1,8.2,4.0 \mathrm{~Hz}, 4 \mathrm{H}), 7.24(\mathrm{~d}, J=$ $6.0 \mathrm{~Hz}, 1 \mathrm{H}) ;{ }^{13} \mathbf{C}$ NMR (101 MHz, $\left.\mathbf{C D C l}_{3}\right): \delta, 191.5,187.5,139.6,139.4,135.9,135.8$, 132.7, 132.2 (2C), 132.1, 131.3, 130.2 (2C), 129.6, 129.3, 128.8, 128.7, 127.7, 125.9, 123.4; HR-MS (ESI, m/z): Calculated for $\mathrm{C}_{20} \mathrm{H}_{14} \mathrm{BrO}_{2}\left([\mathrm{M}+\mathrm{H}]^{+}\right)$: 365.0172 ; Found: 365.0187.

(E)-2-(3-oxopent-1-en-1-yl)benzaldehyde $(6 \mathbf{6 x}): 65 \mathrm{mg}, 55 \%$ yield; $\mathrm{R}_{\mathrm{f}}=0.37(10: 90=$ EtOAc/n-Hexane); Colourless Liquid; FT-IR (neat): 2945, 2854, 1660, 1605, 1263, 1074, 900, $764 \mathrm{~cm}^{-1}$; ${ }^{1} \mathbf{H}$ NMR (400 MHz, $\left.\mathbf{C D C l}_{3}\right): \delta, 10.20$ (s, 1H), 8.43 (d, $J$ $=16.3 \mathrm{~Hz}, 1 \mathrm{H}), 7.82(\mathrm{dd}, J=7.4,1.3 \mathrm{~Hz}, 1 \mathrm{H}), 7.69-7.45(\mathrm{~m}, 3 \mathrm{H}), 6.57$ (d, $J=16.3 \mathrm{~Hz}, 1 \mathrm{H}), 2.74$ (q, $J=7.3 \mathrm{~Hz}, 2 \mathrm{H}), 1.14(\mathrm{t}, J=7.3 \mathrm{~Hz}, 3 \mathrm{H})$;

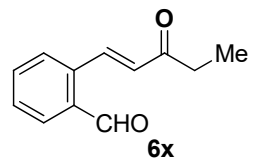

${ }^{13}$ C NMR (101 MHz, $\left.\mathbf{C D C l}_{3}\right): \delta, 201.1,192.4,139.2,136.7,133.9$, 133.9, 133.5, 130.8, 129.9, 127.9, 33.3, 8.1; HR-MS (ESI, m/z): Calculated for $\mathrm{C}_{12} \mathrm{H}_{13} \mathrm{O}_{2}$ $\left([\mathrm{M}+\mathrm{H}]^{+}\right)$: 189.0910; Found: 189.0891 .

\section{Representative synthetic procedure for phthalides:}

\section{Step-I}

A solution of chalcone 6 ( 1 equiv, $0.2 \mathrm{mmol}$ ) and catalyst ( $5 \mathrm{~mol} \%$ ) in toluene was taken in a small round bottom flask with a magnetic bar and was placed over a magnetic stirrer at room temperature. Then $6 \mathrm{M}$ solution of TBHP $(0.1 \mathrm{mmol}, 5$ equiv) was added dropwise to the premix solution. The reaction mixture was allowed to stirred at room temperature for 3-7 $\mathrm{h}$. The reaction was stopped, when all the starting material (chalcone) were consumed (monitored by TLC). Finally, the reaction was filtered with a small plug of silica and washed with dichloromethane as solvent. The peroxy-acetal was directly taken for step-II after removing the solvent with vaccuo. 


\section{Step-II}

The solution of peroxy-acetal (obtained from step-I) in dichloromethane was taken in a small round bottom flask with a bar magnet and was placed over a magnetic stirrer. Solid $\mathrm{FeCl}_{3} \cdot 6 \mathrm{H}_{2} \mathrm{O}(25 \mathrm{~mol} \%)$ was directly added to the flask and the reaction was allowed to stir at room temperature for overnight. Then, the reaction was filtered with a small plug of silica gel using dichloromethane as solvent. After the removal of solvent in vaccuo, the crude phthalides was washed with n-hexane to get optically pure phthalides.

3-(2-Oxo-2-phenylethyl)isobenzofuran-1(3H)-one (7a): (before wash $92 \mathrm{mg}$; 86\% yield; $89 \%$ ee); $62 \mathrm{mg} ; 58 \%$ yield; $\mathrm{R}_{\mathrm{f}}=0.30$ (20:80 = EtOAc/n-Hexane); White Solid; mp $164{ }^{0} \mathrm{C}$; FT-IR (neat): 3020, 2899, 2833, 2351, 1751, 1649, 1284, 1050, $696 \mathrm{~cm}^{-}$ 1, ${ }^{1}$ H NMR (400 MHz, $\left.\mathbf{C D C l}_{3}\right): \delta, 7.97-7.91$ (m, 2H), 7.89 (d, $J=7.6$ $\mathrm{Hz}, 1 \mathrm{H}), 7.67-7.61(\mathrm{~m}, 1 \mathrm{H}), 7.61-7.49(\mathrm{~m}, 3 \mathrm{H}), 7.46$ (t, $J=7.7 \mathrm{~Hz}$,

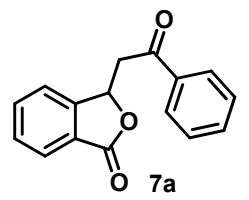
2H), 6.15 (t, $J=6.5 \mathrm{~Hz}, 1 \mathrm{H}), 3.75(\mathrm{dd}, J=17.7,5.8 \mathrm{~Hz}, 1 \mathrm{H}), 3.38$ (dd, $J=17.7,7.2 \mathrm{~Hz}, 1 \mathrm{H})$; ${ }^{13}$ C NMR (101 MHz, $\left.\mathbf{C D C l}_{3}\right): \delta, 196.0,170.2,149.8,136.2,134.3,133.9,129.5,128.8$ (2C), 128.2 (2C), 125.9, 125.7, 122.8, 77.2, 43.7; HR-MS (ESI, m/z): Calculated for $\mathrm{C}_{16} \mathrm{H}_{13} \mathrm{O}_{3}$ $\left([\mathrm{M}+\mathrm{H}]^{+}\right): 253.0859$; Found: $253.0869 ;[\alpha]_{\mathrm{D}}{ }^{20}=-86.32\left(\mathrm{c}=0.5, \mathrm{CHCl}_{3}, 92 \%\right.$ ee $)$.

The enantiomeric ratio was determined by HPLC analysis using Diacel Chiralpak OD-3 column, $\mathrm{n}$-Hexane/2-Propanol $=80 / 20$, flow rate $=1.0 \mathrm{~mL} / \mathrm{min}, \lambda=254 \mathrm{~nm} ; \mathrm{t}_{\mathrm{R}}=14.8 \mathrm{~min}$ (major), $\mathrm{t}_{\mathrm{R}}=18.8 \mathrm{~min}$ (minor).

3-(2-Oxo-2-(p-tolyl)ethyl)isobenzofuran-1(3H)-one (7b): (before wash $80 \mathrm{mg}$; 75\% yield; 91\% ee); $63 \mathrm{mg} ; 59 \%$ yield; $\mathrm{R}_{\mathrm{f}}=0.32$ (20:80 = EtOAc/n-Hexane); White solid; mp $127{ }^{0} \mathrm{C}$; FT-IR (neat): 3079, 3030, 2853, 2786, 2360, 1753, 1659, 1498, 1273, 1078, 756, $653 \mathrm{~cm}^{-1}$; ${ }^{1} \mathbf{H}$ NMR (400 MHz, CDCl3): $\delta, 7.86(\mathrm{t}, J=7.2$ $\mathrm{Hz}, 1 \mathrm{H}), 7.82$ (d, $J=8.0 \mathrm{~Hz}, 2 \mathrm{H}), 7.63$ (t, $J=7.5 \mathrm{~Hz}, 1 \mathrm{H}), 7.58-7.47$ $(\mathrm{m}, 2 \mathrm{H}), 7.29-7.20(\mathrm{~m}, 2 \mathrm{H}), 6.13(\mathrm{t}, J=6.4 \mathrm{~Hz}, 1 \mathrm{H}), 3.71(\mathrm{dd}, J=$

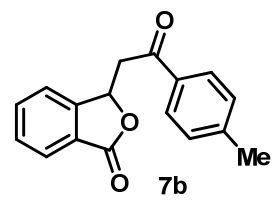
17.5, $5.2 \mathrm{~Hz}, 1 \mathrm{H}), 3.47-3.17(\mathrm{~m}, 1 \mathrm{H}), 2.39(\mathrm{~s}, 3 \mathrm{H}) ;{ }^{13} \mathbf{C}$ NMR (101 MHz, CDCl $\left.\mathbf{3}\right): \delta$, 195.6, 170.2, 149.8, 144.9, 134.3, 133.8, 129.5 (2C), 129.4, 128.3 (2C), 125.9, 125.7, 122.9, 77.3, 43.5, 21.7; HR-MS (ESI, $\mathbf{m} / \mathbf{z})$ : Calculated for $\mathrm{C}_{17} \mathrm{H}_{14} \mathrm{NaO}_{3}\left([\mathrm{M}+\mathrm{Na}]^{+}\right): 289.0835$; Found: $289.0845 ;[\alpha]_{\mathrm{D}}^{22}=-114.02\left(\mathrm{c}=0.5, \mathrm{CHCl}_{3}, 92 \%\right.$ ee $)$. 
The enantiomeric ratio was determined by HPLC analysis using Diacel Chiralpak OD-3 column, $\mathrm{n}$-Hexane/2-Propanol $=80 / 20$, flow rate $=1.0 \mathrm{~mL} / \mathrm{min}, \lambda=254 \mathrm{~nm} ; \mathrm{t}_{\mathrm{R}}=12.8 \mathrm{~min}$ (major), $\mathrm{t}_{\mathrm{R}}=16.7 \mathrm{~min}$ (minor).

3-(2-(4-Methoxyphenyl)-2-oxoethyl)isobenzofuran-1(3H)-one (7c): (before wash $72 \mathrm{mg}$; $68 \%$ yield; 90\% ee); $61 \mathrm{mg} ; 57 \%$ yield; $\mathrm{R}_{\mathrm{f}}=0.18(20: 80=\mathrm{EtOAc} / \mathrm{n}-$ Hexane); White solid; mp $133{ }^{0} \mathrm{C}$; FT-IR (neat): 3048, 3018, 2878, 2829, 2359, 2329, 1644, 1452, 756, $695 \mathrm{~cm}^{-1}$; ${ }^{1}$ H NMR (400 MHz,

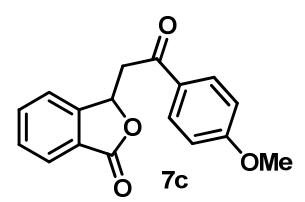
CDCI3): $\delta, 7.88(\mathrm{dd}, J=12.6,8.4 \mathrm{~Hz}, 3 \mathrm{H}), 7.62(\mathrm{t}, J=7.4 \mathrm{~Hz}, 1 \mathrm{H})$, $7.57-7.45$ (m, 2H), 6.91 (d, $J=8.8 \mathrm{~Hz}, 2 \mathrm{H}), 6.13$ (t, $J=6.5 \mathrm{~Hz}, 1 \mathrm{H}), 3.84$ (s, 3H), 3.68 (dd, $J=17.4,5.8 \mathrm{~Hz}, 1 \mathrm{H}), 3.31(\mathrm{dd}, J=17.4,7.3 \mathrm{~Hz}, 1 \mathrm{H}) ;{ }^{13} \mathbf{C}$ NMR (101 MHz, CDCl $\left.\mathbf{3}\right): \delta$, $194.4,170.2$, 164.1, 149.9, 134.3, 130.5 (2C), 129.4, 129.3, 125.9, 125.7, 122.9, 113.9 (2C), 77.4, 55.6, 43.3; HR-MS (ESI, $\mathbf{m} / \mathbf{z})$ : Calculated for $\mathrm{C}_{17} \mathrm{H}_{15} \mathrm{O}_{4}\left([\mathrm{M}+\mathrm{H}]^{+}\right)$: 283.0965; Found: $283.0980 ;[\alpha]_{\mathrm{D}}^{22}=-80.5\left(\mathrm{c}=0.5, \mathrm{CHCl}_{3}, 94 \%\right.$ ee $)$.

The enantiomeric ratio was determined by HPLC analysis using Diacel Chiralpak OD-3 column, $n$-Hexane/2-Propanol $=75 / 25$, flow rate $=1.0 \mathrm{~mL} / \mathrm{min}, \lambda=254 \mathrm{~nm} ; \mathrm{t}_{\mathrm{R}}=15.0 \mathrm{~min}$ (major), $\mathrm{t}_{\mathrm{R}}=19.9 \mathrm{~min}$ (minor).

3-(2-(4-Chlorophenyl)-2-oxoethyl)isobenzofuran-1(3H)-one (7d): (before wash $98 \mathrm{mg}$; 92\% yield; 92\% ee); $51 \mathrm{mg} ; 48 \%$ yield; $\mathrm{R}_{\mathrm{f}}=0.31(20: 80=$ EtOAc $/ \mathrm{n}-$ Hexane); White Solid; mp $168{ }^{0} \mathrm{C}$; FT-IR (neat): 3022, 2905, 2835, 2360, 1645, $698 \mathrm{~cm}^{-1} ;{ }^{1} \mathbf{H}$ NMR (400 MHz, $\left.\mathbf{C D C l}_{3}\right): \delta, 7.95-7.83(\mathrm{~m}$,

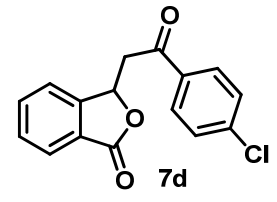
$3 \mathrm{H}), 7.72-7.59(\mathrm{~m}, 1 \mathrm{H}), 7.53(\mathrm{t}, J=7.6 \mathrm{~Hz}, 2 \mathrm{H}), 7.45(\mathrm{dd}, J=16.6,5.3 \mathrm{~Hz}, 2 \mathrm{H}), 6.13(\mathrm{t}, J=$ $6.5 \mathrm{~Hz}, 1 \mathrm{H}), 3.70(\mathrm{dd}, J=17.6,6.0 \mathrm{~Hz}, 1 \mathrm{H}), 3.36(\mathrm{dd}, J=17.6,6.9 \mathrm{~Hz}, 1 \mathrm{H}) ;{ }^{13} \mathbf{C} \mathbf{N M R}(\mathbf{1 0 1}$ MHz, $\left.\mathbf{C D C l}_{3}\right): \delta, 194.8,170.0,149.5,140.4,134.5,134.3,129.6$ (2C), 129.5, 129.2 (2C), 125.9, 125.8, 122.7, 77.0, 43.6; HR-MS (ESI, m/z): Calculated for $\mathrm{C}_{16} \mathrm{H}_{12} \mathrm{ClO}_{3}\left([\mathrm{M}+\mathrm{H}]^{+}\right)$: 287.0469; Found: 287.0460; $[\alpha]_{\mathrm{D}}{ }^{18}=-49.84\left(\mathrm{c}=0.5, \mathrm{CHCl}_{3}, 97 \%\right.$ ee $)$.

The enantiomeric ratio was determined by HPLC analysis using Diacel Chiralpak OD-3 column, $\mathrm{n}$-Hexane $/ 2$-Propanol $=80 / 20$, flow rate $=1.0 \mathrm{~mL} / \mathrm{min}, \lambda=254 \mathrm{~nm} ; \mathrm{t}_{\mathrm{R}}=18.5 \mathrm{~min}$ (major), $\mathrm{t}_{\mathrm{R}}=27.5 \mathrm{~min}$ (minor).

3-(2-(4-Bromophenyl)-2-oxoethyl)isobenzofuran-1(3H)-one (7e): (before wash $92 \mathrm{mg}$; 91\% yield; 91\% ee); $60 \mathrm{mg} ; 57 \%$ yield; $\mathrm{R}_{\mathrm{f}}=0.30$ (20:80 = EtOAc/n-Hexane); White Solid; mp $164{ }^{0} \mathrm{C}$; FT-IR (neat): 3025, 2849, 2794, 2351, 2327, 1739, 1664,

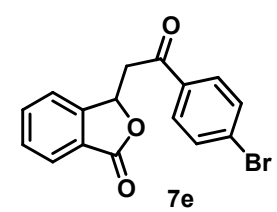


1503, 1452, 1267, 1013, 747, $696 \mathrm{~cm}^{-1}$; ${ }^{1}$ H NMR (400 MHz, CDCl3): $\delta, 7.88$ (t, $J=11.0$ $\mathrm{Hz}, 1 \mathrm{H}), 7.78$ (t, $J=14.9 \mathrm{~Hz}, 2 \mathrm{H}), 7.72-7.57$ (m, 3H), 7.51 (dd, $J=22.7,16.0 \mathrm{~Hz}, 2 \mathrm{H}), 6.13$ $(\mathrm{t}, J=6.4 \mathrm{~Hz}, 1 \mathrm{H}), 3.93-3.56(\mathrm{~m}, 1 \mathrm{H}), 3.47-3.11(\mathrm{~m}, 1 \mathrm{H}) ;{ }^{13} \mathbf{C}$ NMR (101 MHz, CDCl$\left.)_{3}\right)$ $\delta, 195.0,170.0,149.5,134.9,134.3,132.2$ (2C), 129.7 (2C), 129.5, 129.2, 125.9, 125.8, 122.7, 76.9, 43.6; HR-MS (ESI, $\boldsymbol{m} / \mathbf{z})$ : Calculated for $\mathrm{C}_{16} \mathrm{H}_{11} \mathrm{BrNaO}_{3}\left([\mathrm{M}+\mathrm{Na}]^{+}\right)$: 352.9784 ; Found: $352.9766 ;[\alpha]_{\mathrm{D}}^{23}=-54.78\left(\mathrm{c}=0.5, \mathrm{CHCl}_{3},>99 \%\right.$ ee $)$.

The enantiomeric ratio was determined by HPLC analysis using Diacel Chiralpak OD-3 column, $n$-Hexane/2-Propanol $=75 / 25$, flow rate $=1.0 \mathrm{~mL} / \mathrm{min}, \lambda=254 \mathrm{~nm} ; \mathrm{t}_{\mathrm{R}}=16.4 \mathrm{~min}$ (major), $\mathrm{t}_{\mathrm{R}}=23.7 \mathrm{~min}$ (minor).

3-(2-(2-Bromophenyl)-2-oxoethyl)isobenzofuran-1(3H)-one (7f): (before wash $82 \mathrm{mg}$; $78 \%$ yield; $50 \%$ ee); $30 \mathrm{mg} ; 28 \%$ yield; $\mathrm{R}_{\mathrm{f}}=0.32$ (20:80 = EtOAc/n-Hexane); White Solid; mp $120{ }^{0} \mathrm{C}$; FT-IR (neat): 3083, 3025, 2951, 2871, 2360, 1771, 1702, 1464, 1290, 1211, 1069, 752, $692 \mathrm{~cm}^{-1}$; ${ }^{1}$ H NMR (400 MHz, CDC13): $\delta, 7.89(\mathrm{~d}, J=7.6 \mathrm{~Hz}, 1 \mathrm{H}), 7.67(\mathrm{t}, J=7.4 \mathrm{~Hz}, 1 \mathrm{H}), 7.56(\mathrm{dt}, J=15.1$,

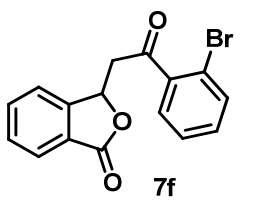
$9.1 \mathrm{~Hz}, 3 \mathrm{H}), 7.47$ (d, $J=7.5 \mathrm{~Hz}, 1 \mathrm{H}), 7.38$ (t, $J=7.5 \mathrm{~Hz}, 1 \mathrm{H}), 7.31$ (t, $J=7.2 \mathrm{~Hz}, 1 \mathrm{H}), 6.10$ (t, $J=6.4 \mathrm{~Hz}, 1 \mathrm{H}), 3.63(\mathrm{dd}, J=17.6,6.6 \mathrm{~Hz}, 1 \mathrm{H}), 3.46(\mathrm{dd}, J=17.6,6.4 \mathrm{~Hz}, 1 \mathrm{H}) ;{ }^{13} \mathbf{C ~ N M R}$ (101 MHz, $\left.\mathbf{C D C l}_{3}\right): \delta, 199.5,169.9,149.2,140.2,134.3,133.9,132.4,129.5,129.2,127.7$, 125.9, 125.8, 122.6, 118.9, 77.0, 47.4; HR-MS (ESI, m/z): Calculated for $\mathrm{C}_{16} \mathrm{H}_{11} \mathrm{BrNaO}_{3}$ $\left([\mathrm{M}+\mathrm{Na}]^{+}\right): 352.9784$; Found: $352.9767 ;[\alpha]_{\mathrm{D}}{ }^{22}=+10.24\left(\mathrm{c}=0.5, \mathrm{CHCl}_{3}, 92 \%\right.$ ee $)$.

The enantiomeric ratio was determined by HPLC analysis using Diacel Chiralpak OD-3 column, $\mathrm{n}$-Hexane/2-Propanol $=75 / 25$, flow rate $=1.0 \mathrm{~mL} / \mathrm{min}, \lambda=254 \mathrm{~nm} ; \mathrm{t}_{\mathrm{R}}=12.8 \mathrm{~min}$ (major), $\mathrm{t}_{\mathrm{R}}=22.9 \mathrm{~min}$ (minor).

3-(2-(4-Iodophenyl)-2-oxoethyl)isobenzofuran-1(3H)-one (7g): $15 \mathrm{mg} ; 71 \%$ yield; $\mathrm{R}_{\mathrm{f}}=$ $0.31\left(20: 80=\right.$ EtOAc/n-Hexane); White Solid; mp $182{ }^{0} \mathrm{C}$; FT-IR (neat): 3079, 3024, 2876, 2839, 2359, 2340, 1738, 1652, 1454, 701 $\mathrm{cm}^{-1} ;{ }^{1} \mathbf{H}$ NMR (400 MHz, $\left.\mathbf{C D C l}_{3}\right): \delta, 7.90(\mathrm{~d}, J=7.8 \mathrm{~Hz}, 1 \mathrm{H}), 7.84$

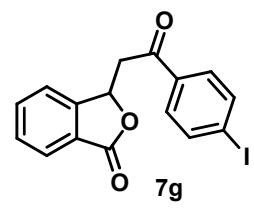
$(\mathrm{d}, J=8.3 \mathrm{~Hz}, 2 \mathrm{H}), 7.65(\mathrm{t}, J=7.7 \mathrm{~Hz}, 3 \mathrm{H}), 7.54(\mathrm{dd}, J=7.1,5.4 \mathrm{~Hz}, 2 \mathrm{H}), 6.13(\mathrm{t}, J=6.4$ $\mathrm{Hz}, 1 \mathrm{H}), 3.70(\mathrm{dd}, J=17.6,5.9 \mathrm{~Hz}, 1 \mathrm{H}), 3.33(\mathrm{dd}, J=17.6,7.0 \mathrm{~Hz}, 1 \mathrm{H}) ;{ }^{13} \mathbf{C}$ NMR (101 MHz, $\left.\mathbf{C D C l}_{3}\right): \delta, 195.4,170.0,149.5,138.2$ (2C), 135.4, 134.3, 129.5, 129.5 (2C), 125.9, 125.8, 122.7, 102.1, 76.9, 43.6; HR-MS (ESI, m/z): Calculated for $\mathrm{C}_{16} \mathrm{H}_{12} \mathrm{IO}_{3}\left([\mathrm{M}+\mathrm{H}]^{+}\right)$: 378.9826; Found: $378.9837 ;[\alpha]_{\mathrm{D}}^{22}=-9.32\left(\mathrm{c}=0.5, \mathrm{CHCl}_{3}, 91 \%\right.$ ee $)$. 
The enantiomeric ratio was determined by HPLC analysis using Diacel Chiralpak OD-3 column, $\mathrm{n}$-Hexane/2-Propanol $=75 / 25$, flow rate $=1.0 \mathrm{~mL} / \mathrm{min}, \lambda=254 \mathrm{~nm} ; \mathrm{t}_{\mathrm{R}}=22.0 \mathrm{~min}$ (major), $\mathrm{t}_{\mathrm{R}}=28.7 \mathrm{~min}$ (minor).

3-(2-(4-Fluorophenyl)-2-oxoethyl)isobenzofuran-1(3H)-one (7h): (before wash $75 \mathrm{mg}$; $70 \%$ yield; 92\% ee); $60 \mathrm{mg} ; 56 \%$ yield; $\mathrm{R}_{\mathrm{f}}=0.26(20: 80=\mathrm{EtOAc} / \mathrm{n}-$ Hexane); Grey solid; mp $162{ }^{0} \mathrm{C}$; FT-IR (neat): 2989, 2835, 2360, 2334, 1666, 1449, $695 \mathrm{~cm}^{-1}$; ${ }^{1}$ H NMR (400 MHz, CDC13): $\delta, 7.96$

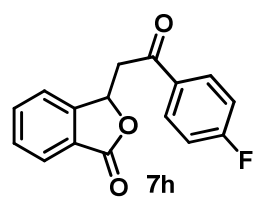
$(\mathrm{dd}, J=8.6,5.4 \mathrm{~Hz}, 2 \mathrm{H}), 7.87$ (d, $J=7.6 \mathrm{~Hz}, 1 \mathrm{H}), 7.64$ (t, $J=7.5 \mathrm{~Hz}$, 1H), $7.52(\mathrm{dd}, J=12.5,7.4 \mathrm{~Hz}, 2 \mathrm{H}), 7.12(\mathrm{t}, J=8.4 \mathrm{~Hz}, 2 \mathrm{H}), 6.12(\mathrm{t}, J=6.4 \mathrm{~Hz}, 1 \mathrm{H}), 3.70$ $(\mathrm{dd}, J=17.6,6.0 \mathrm{~Hz}, 1 \mathrm{H}), 3.36(\mathrm{dd}, J=17.6,6.9 \mathrm{~Hz}, 1 \mathrm{H}) ;{ }^{13} \mathbf{C}$ NMR $\left(\mathbf{1 0 1} \mathbf{M H z}, \mathbf{C D C l}_{3}\right): \delta$, 194.42, 170.07, 166.15 (d, $J=256.2 \mathrm{~Hz}), 149.60,134.3$ (2C), 132.7 (d, $J=3.0 \mathrm{~Hz}), 130.92$ (d, $J=9.5 \mathrm{~Hz}), 129.50,125.87,125.77,122.74,116.1,115.9,77.07,43.58$; HR-MS (ESI, $\boldsymbol{m} / \mathbf{z})$ : Calculated for $\mathrm{C}_{16} \mathrm{H}_{11} \mathrm{FNaO}_{3}\left([\mathrm{M}+\mathrm{Na}]^{+}\right)$: 293.0584; Found: 293.0604; $[\alpha]_{\mathrm{D}}^{22}=-100.2(\mathrm{c}$ $=0.5, \mathrm{CHCl}_{3}, 96 \%$ ee).

The enantiomeric ratio was determined by HPLC analysis using Diacel Chiralpak OD-3 column, $n$-Hexane/2-Propanol $=80 / 20$, flow rate $=1.0 \mathrm{~mL} / \mathrm{min}, \lambda=254 \mathrm{~nm} ; \mathrm{t}_{\mathrm{R}}=14.7 \mathrm{~min}$ (major), $\mathrm{t}_{\mathrm{R}}=21.8 \mathrm{~min}$ (minor).

3-(2-(2,4-Difluorophenyl)-2-oxoethyl)isobenzofuran-1(3H)-one (7i): (before wash $95 \mathrm{mg}$; $89 \%$ yield; 87\% ee); $70 \mathrm{mg} ; 66 \%$ yield; $\mathrm{R}_{\mathrm{f}}=0.30(20: 80=\mathrm{EtOAc} / \mathrm{n}-$ Hexane); Violet solid; mp $173{ }^{0} \mathrm{C}$; FT-IR (neat): 3014, 2862, 2817, 2360, 1740, 1667, 1502, 1258, 988, $694 \mathrm{~cm}^{-1} ;{ }^{1} \mathbf{H}$ NMR (400 MHz, CDC13): $\delta, 7.91(\mathrm{~s}, 1 \mathrm{H}), 7.70(\mathrm{dd}, J=49.3,27.8 \mathrm{~Hz}, 3 \mathrm{H}), 7.40(\mathrm{~d}, J=$

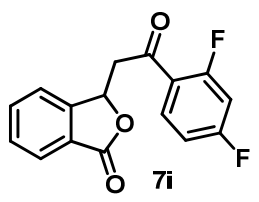
$122.5 \mathrm{~Hz}, 3 \mathrm{H}), 6.14$ (s, 1H), 3.69 (s, 1H), 3.36 (s, 1H); ${ }^{13}$ C NMR (101 MHz, CDC13): $\delta$, 193.6, 170.3, 154.1 (d, $J=270.7 \mathrm{~Hz}), 151.8$ (d, $J=13.6 \mathrm{~Hz}), 149.4,134.5,133.3,129.7$ (2C), 125.9, $125.6(\mathrm{~d}, J=7.6 \mathrm{~Hz}), 122.8,118.1,117.9$ (d, $J=17.9 \mathrm{~Hz}), 117.6(\mathrm{~d}, J=18.2 \mathrm{~Hz})$, 77.0, 43.9; HR-MS (ESI, m/z): Calculated for $\mathrm{C}_{16} \mathrm{H}_{11} \mathrm{~F}_{2} \mathrm{O}_{3}\left([\mathrm{M}+\mathrm{H}]^{+}\right)$: 289.0671; Found: $289.0697 ;[\alpha]_{\mathrm{D}}^{20}=-48.82\left(\mathrm{c}=0.5, \mathrm{CHCl}_{3}, 95 \%\right.$ ee $)$.

The enantiomeric ratio was determined by HPLC analysis using Diacel Chiralpak OD-3 column, $\mathrm{n}$-Hexane/2-Propanol $=80 / 20$, flow rate $=1.0 \mathrm{~mL} / \mathrm{min}, \lambda=254 \mathrm{~nm} ; \mathrm{t}_{\mathrm{R}}=16.6 \mathrm{~min}$ (major), $\mathrm{t}_{\mathrm{R}}=25.7 \mathrm{~min}$ (minor). 
3-(2-(4-Nitrophenyl)-2-oxoethyl)isobenzofuran-1(3H)-one (7j): (before wash $66 \mathrm{mg}$; 62\% yield; 84\% ee); $44 \mathrm{mg} ; 41 \%$ yield; $\mathrm{R}_{\mathrm{f}}=0.20$ (20:80 = EtOAc/n-Hexane); Brown solid; $\mathrm{mp}$ $209{ }^{0} \mathrm{C}$; FT-IR (neat): 3028, 2924, 2857, 2817, 2272, 1745, 1655, 1491, 1452, 1072, 910, $700 \mathrm{~cm}^{-1} ;{ }^{1} \mathbf{H}$ NMR (400 MHz, CDCl3): $\delta, 8.33(\mathrm{~d}, J=8.6 \mathrm{~Hz}, 2 \mathrm{H})$, $8.18-8.05(\mathrm{~m}, 2 \mathrm{H}), 7.93(\mathrm{~d}, J=7.6 \mathrm{~Hz}, 1 \mathrm{H}), 7.77-7.63(\mathrm{~m}, 1 \mathrm{H}), 7.48$ $(\mathrm{dt}, J=21.3,11.7 \mathrm{~Hz}, 2 \mathrm{H}), 6.15(\mathrm{t}, J=6.3 \mathrm{~Hz}, 1 \mathrm{H}), 3.77(\mathrm{dd}, J=17.7$,

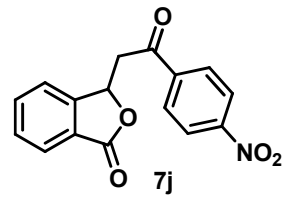
6.2 Hz, 1H), 3.45 (dd, $J=17.7,6.5 \mathrm{~Hz}, 1 \mathrm{H}) ;{ }^{13} \mathbf{C}$ NMR (101 MHz, CDCl3): $\delta, 194.5,169.8$, $150.7,149.1,140.4,134.4,129.7,129.3$ (2C), 126.0, 125.9, 124.1 (2C), 122.5, 76.6, 44.2; HR-MS (ESI, $\boldsymbol{m} / \mathbf{z})$ : Calculated for $\mathrm{C}_{16} \mathrm{H}_{12} \mathrm{NO}_{5}\left([\mathrm{M}+\mathrm{H}]^{+}\right)$: 298.0710; Found: 298.0704; $[\alpha]_{\mathrm{D}}{ }^{24}$ $=-29.8\left(\mathrm{c}=0.5, \mathrm{CHCl}_{3}, 84 \%\right.$ ee $)$.

The enantiomeric ratio was determined by HPLC analysis using Diacel Chiralpak OD-3 column, $\mathrm{n}$-Hexane/2-Propanol $=65 / 35$, flow rate $=1.2 \mathrm{~mL} / \mathrm{min}, \lambda=254 \mathrm{~nm} ; \mathrm{t}_{\mathrm{R}}=23.6 \mathrm{~min}$ (major), $\mathrm{t}_{\mathrm{R}}=38.5 \mathrm{~min}$ (minor).

3-(2-(3-Nitrophenyl)-2-oxoethyl)isobenzofuran-1(3H)-one (7k): (before wash $78 \mathrm{mg} ; 73 \%$ yield; 88\% ee); $64 \mathrm{mg} ; 60 \%$ yield; $\mathrm{R}_{\mathrm{f}}=0.17$ (20:80 = EtOAc/n-Hexane); Brown solid; $\mathrm{mp}$ $178{ }^{0} \mathrm{C}$; FT-IR (neat): 3081, 3024, 2843, 2360, 2338, 1741, 1671, 1500, 1452, 1263, 1031, 759, $649 \mathrm{~cm}^{-1}$; ${ }^{\mathbf{1}} \mathbf{H}$ NMR (400 MHz, CDCl3): $\delta, 8.75(\mathrm{~s}, 1 \mathrm{H}), 8.42(\mathrm{t}, J=13.1 \mathrm{~Hz}, 1 \mathrm{H}), 8.28(\mathrm{~d}, J=7.7$

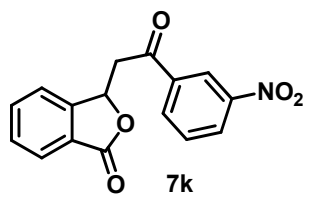
$\mathrm{Hz}, 1 \mathrm{H}), 7.91$ (d, $J=7.8 \mathrm{~Hz}, 1 \mathrm{H}), 7.70(\mathrm{dd}, J=17.7,7.9 \mathrm{~Hz}, 2 \mathrm{H}), 7.61-7.46(\mathrm{~m}, 2 \mathrm{H}), 6.16$ $(\mathrm{t}, J=6.3 \mathrm{~Hz}, 1 \mathrm{H}), 3.77(\mathrm{dd}, J=17.7,6.4 \mathrm{~Hz}, 1 \mathrm{H}), 3.48(\mathrm{dd}, J=17.7,6.3 \mathrm{~Hz}, 1 \mathrm{H}) ;{ }^{13} \mathbf{C} \mathbf{~ N M R}$

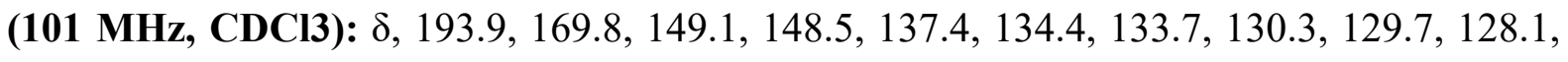
125.96, 125.91, 123.1, 122.5, 76.6, 43.9; HR-MS (ESI, m/z): Calculated for $\mathrm{C}_{16} \mathrm{H}_{12} \mathrm{NO}_{5}$ $\left([\mathrm{M}+\mathrm{H}]^{+}\right): 298.0710$; Found: $298.0704 ;[\alpha]_{\mathrm{D}}{ }^{20}=-44.08\left(\mathrm{c}=0.5, \mathrm{CHCl}_{3}, 91 \%\right.$ ee $)$.

The enantiomeric ratio was determined by HPLC analysis using Diacel Chiralpak OD-3 column, $\mathrm{n}$-Hexane/2-Propanol $=65 / 35$, flow rate $=1.2 \mathrm{~mL} / \mathrm{min}, \lambda=254 \mathrm{~nm} ; \mathrm{t}_{\mathrm{R}}=16.3 \mathrm{~min}$ (major), $\mathrm{t}_{\mathrm{R}}=23.4 \mathrm{~min}$ (minor).

3-(2-(Furan-2-yl)-2-oxoethyl)isobenzofuran-1(3H)-one (7l): (before wash $100 \mathrm{mg}$; 93\% yield; 82\% ee); 70 mg; 65\% yield; $\mathrm{R}_{\mathrm{f}}=0.19$ (20:80 = EtOAc/n-Hexane); Grey Solid; mp 106 ${ }^{0}$ C; FT-IR (neat): 3129, 3015, 2908, 2836, 2358, 1761, 1672, 1469, 1293, 1061, 987, 748, $701 \mathrm{~cm}^{-1}$; ${ }^{1} \mathbf{H}$ NMR (400 MHz, $\left.\mathbf{C D C l}_{3}\right): \delta, 7.87(\mathrm{t}, J=$ $10.0 \mathrm{~Hz}, 1 \mathrm{H}), 7.64$ (t, $J=7.5 \mathrm{~Hz}, 1 \mathrm{H}), 7.59$ (s, 1H), 7.52 (t, $J=6.8 \mathrm{~Hz}$,

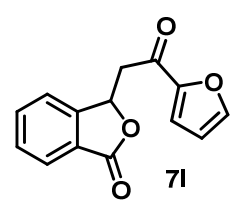


2H), $7.25(\mathrm{t}, J=8.2 \mathrm{~Hz}, 1 \mathrm{H}), 6.56(\mathrm{t}, J=7.5 \mathrm{~Hz}, 1 \mathrm{H}), 6.09(\mathrm{t}, J=6.6 \mathrm{~Hz}, 1 \mathrm{H}), 3.56(\mathrm{dd}, J=$ 17.1, 6.6 Hz, 1H), 3.25 (dd, $J=17.1,6.6 \mathrm{~Hz}, 1 \mathrm{H}) ;{ }^{13} \mathbf{C}$ NMR (101 $\left.\mathbf{M H z}, \mathbf{C D C l}_{3}\right): \delta, 184.6$, $170.0,152.2$, 149.4, 147.1, 134.3, 129.5, 125.9, 125.8, 122.6, 118.2, 112.7, 76.8, 43.4; HRMS (ESI, m/z): Calculated for $\mathrm{C}_{14} \mathrm{H}_{11} \mathrm{O}_{4}\left([\mathrm{M}+\mathrm{H}]^{+}\right)$: 243.0652; Found: $243.0674 ;[\alpha]_{\mathrm{D}}{ }^{19}=-$ $25.66\left(\mathrm{c}=0.5, \mathrm{CHCl}_{3}, 83 \%\right.$ ee $)$.

The enantiomeric ratio was determined by HPLC analysis using Diacel Chiralpak OD-3 column, $\mathrm{n}$-Hexane/2-Propanol $=75 / 25$, flow rate $=1.0 \mathrm{~mL} / \mathrm{min}, \lambda=254 \mathrm{~nm} ; \mathrm{t}_{\mathrm{R}}=10.9 \mathrm{~min}$ (major), $\mathrm{t}_{\mathrm{R}}=13.9 \mathrm{~min}$ (minor).

3-(2-Oxo-2-(thiophen-2-yl)ethyl)isobenzofuran-1(3H)-one (7m): (before wash $99 \mathrm{mg}$; 92\% yield; $88 \%$ ee); $70 \mathrm{mg} ; 65 \%$ yield; $\mathrm{R}_{\mathrm{f}}=0.22$ (20:80 = EtOAc/n-Hexane); White Solid; mp $157{ }^{0} \mathrm{C}$; FT-IR (neat): 3009, 2895, 2833, 1640, 1496, $694 \mathrm{~cm}^{-1} ;{ }^{1} \mathbf{H}$ NMR (400 MHz, $\left.\mathbf{C D C l}_{3}\right): \delta, 7.89(\mathrm{~d}, J=7.6 \mathrm{~Hz}, 1 \mathrm{H}), 7.68$

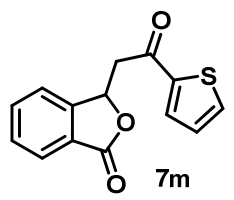
$(\mathrm{t}, J=4.0 \mathrm{~Hz}, 2 \mathrm{H}), 7.64(\mathrm{dd}, J=11.4,4.4 \mathrm{~Hz}, 1 \mathrm{H}), 7.57-7.50(\mathrm{~m}, 2 \mathrm{H}), 7.11(\mathrm{dd}, J=10.6$, $6.2 \mathrm{~Hz}, 1 \mathrm{H}), 6.17-6.01(\mathrm{~m}, 1 \mathrm{H}), 3.65(\mathrm{dd}, J=17.0,6.3 \mathrm{~Hz}, 1 \mathrm{H}), 3.31$ (dt, $J=16.0,8.0 \mathrm{~Hz}$, 1H); ${ }^{13} \mathbf{C}$ NMR (101 MHz, $\left.\mathbf{C D C l}_{3}\right): \delta, 188.6,170.0,149.4,143.4,134.9,134.4,132.9,129.5$, 128.5, 125.8, 125.8, 122.7, 77.0, 44.2; HR-MS (ESI, m/z): Calculated for $\mathrm{C}_{14} \mathrm{H}_{11} \mathrm{O}_{3} \mathrm{~S}$ $\left([\mathrm{M}+\mathrm{H}]^{+}\right): 259.0423$; Found: $259.0423 ;[\alpha]_{\mathrm{D}}{ }^{21}=-86.67\left(\mathrm{c}=0.5, \mathrm{CHCl}_{3}, 94 \%\right.$ ee $)$.

The enantiomeric ratio was determined by HPLC analysis using Diacel Chiralpak OD-3 column, $\mathrm{n}$-Hexane/2-Propanol $=75 / 25$, flow rate $=1.0 \mathrm{~mL} / \mathrm{min}, \lambda=254 \mathrm{~nm} ; \mathrm{t}_{\mathrm{R}}=13.0 \mathrm{~min}$ (major), $\mathrm{t}_{\mathrm{R}}=17.0 \mathrm{~min}$ (minor).

3-(2-(4-Bromophenyl)-2-oxoethyl)-6-methoxyisobenzofuran-1(3H)-one $\quad(7 \mathrm{n}): \quad$ (before wash $82 \mathrm{mg} ; 78 \%$ yield; 87\% ee); $60 \mathrm{mg} ; 57 \%$ yield; $\mathrm{R}_{\mathrm{f}}=0.24$ (20:80 = EtOAc/n-Hexane); Light Yellow solid; mp $155{ }^{0} \mathrm{C}$; FT-IR (neat): 2977, 2915, 2808, 2365, 2337, 1635, 1517, 1453, 1386, 1116, 758, $702 \mathrm{~cm}^{-1}$; ${ }^{1} \mathbf{H}$ NMR (400 MHz, CDCl3): $\delta, 7.79(\mathrm{~d}, J=8.6 \mathrm{~Hz}, 2 \mathrm{H}), 7.66-7.56(\mathrm{~m}$, $2 \mathrm{H}), 7.40(\mathrm{t}, J=9.0 \mathrm{~Hz}, 1 \mathrm{H}), 7.30(\mathrm{~d}, J=2.2 \mathrm{~Hz}, 1 \mathrm{H}), 7.18(\mathrm{dt}, J=$

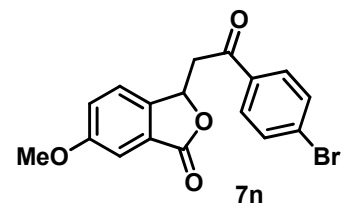
13.3, $6.7 \mathrm{~Hz}, 1 \mathrm{H}), 6.05$ (t, $J=6.5 \mathrm{~Hz}, 1 \mathrm{H}), 3.84(\mathrm{~s}, 3 \mathrm{H}), 3.68(\mathrm{dd}, J=17.6,5.9 \mathrm{~Hz}, 1 \mathrm{H}), 3.30$ $(\mathrm{dd}, J=17.6,7.1 \mathrm{~Hz}, 1 \mathrm{H}) ;{ }^{13} \mathbf{C}$ NMR (101 MHz, CDCl3): $\delta, 195.2,170.1,160.9,141.9$, 134.9, 132.2(2C), 129.6(2C), 129.2, 127.3, 123.7, 123.1, 107.6, 76.9, 55.8, 43.8; HR-MS (ESI, m/z): Calculated for $\mathrm{C}_{17} \mathrm{H}_{13} \mathrm{BrNaO}_{4}\left([\mathrm{M}+\mathrm{Na}]^{+}\right)$: 382.9889 ; Found: $382.9872 ;[\alpha]_{\mathrm{D}}{ }^{19}=-$ $47.78\left(\mathrm{c}=0.5, \mathrm{CHCl}_{3}, 93 \%\right.$ ee $)$. 
The enantiomeric ratio was determined by HPLC analysis using Diacel Chiralpak OD-3 column, $\mathrm{n}$-Hexane/2-Propanol $=80 / 20$, flow rate $=1.0 \mathrm{~mL} / \mathrm{min}, \lambda=254 \mathrm{~nm} ; \mathrm{t}_{\mathrm{R}}=22.7 \mathrm{~min}$ (major), $\mathrm{t}_{\mathrm{R}}=26.9 \mathrm{~min}$ (minor).

6-Methoxy-3-(2-oxo-2-(p-tolyl)ethyl)isobenzofuran-1(3H)-one (7o): (before wash $80 \mathrm{mg}$; 75\% yield; 89\% ee); $61 \mathrm{mg} ; 57 \%$ yield; $\mathrm{R}_{\mathrm{f}}=0.22$ (20:80 = EtOAc/n-Hexane); White solid; mp $135{ }^{0} \mathrm{C}$; FT-IR (neat): 3081, 3059, 2923, 2356, 2334, 1736, 1642, 1491, $695 \mathrm{~cm}^{-1}$; ${ }^{1}$ H NMR (400 MHz, CDCI3): $\delta, 7.82(\mathrm{~d}, J=$ $8.1 \mathrm{~Hz}, 2 \mathrm{H}), 7.41(\mathrm{t}, J=11.6 \mathrm{~Hz}, 1 \mathrm{H}), 7.28(\mathrm{~d}, J=11.0 \mathrm{~Hz}, 1 \mathrm{H}), 7.27$ - $7.21(\mathrm{~m}, 2 \mathrm{H}), 7.17(\mathrm{dd}, J=8.4,2.2 \mathrm{~Hz}, 1 \mathrm{H}), 6.06(\mathrm{t}, J=6.5 \mathrm{~Hz}$,

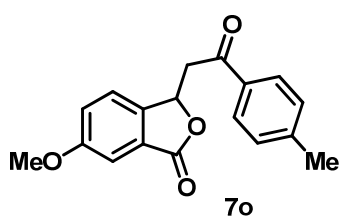
1H), $3.83(\mathrm{~s}, 3 \mathrm{H}), 3.69(\mathrm{dt}, J=13.6,6.8 \mathrm{~Hz}, 1 \mathrm{H}), 3.30(\mathrm{dd}, J=17.5,7.5 \mathrm{~Hz}, 1 \mathrm{H}), 2.39$ (s, 3H); ${ }^{13} \mathbf{C}$ NMR (101 MHz, $\left.\mathbf{C D C l}_{3}\right)$ : $\delta, 195.8,170.3,160.8,144.8,142.3,133.8,129.5$ (2C), 128.3 (2C), 127.3, 123.8, 123.0, 107.5, 77.3, 55.8, 43.7, 21.7; HR-MS (ESI, $\mathbf{m} / \mathbf{z}$ ): Calculated for $\mathrm{C}_{18} \mathrm{H}_{16} \mathrm{NaO}_{4}\left([\mathrm{M}+\mathrm{Na}]^{+}\right): 319.0941$; Found: $319.0959 ;[\alpha]_{\mathrm{D}}{ }^{18}=-85.5\left(\mathrm{c}=0.5, \mathrm{CHCl}_{3}, 94 \%\right.$ ee).

The enantiomeric ratio was determined by HPLC analysis using Diacel Chiralpak OD-3 column, $n$-Hexane/2-Propanol $=80 / 20$, flow rate $=1.0 \mathrm{~mL} / \mathrm{min}, \lambda=254 \mathrm{~nm} ; \mathrm{t}_{\mathrm{R}}=13.0 \mathrm{~min}$ (major), $\mathrm{t}_{\mathrm{R}}=15.2 \mathrm{~min}$ (minor).

\section{6-(Benzyloxy)-3-(2-(4-bromophenyl)-2-oxoethyl)-5-methoxyisobenzofuran-1(3H)-one}

(7p): (before wash $92 \mathrm{mg}$; 89\% yield; 85\% ee); $51 \mathrm{mg}$; 49\% yield; $\mathrm{R}_{\mathrm{f}}$ $=0.24\left(20: 80=\right.$ EtOAc/n-Hexane); Dark Brown solid; mp $168{ }^{0} \mathrm{C}$; FT-IR (neat): 3020, 2991, 2882, 2825, 2359, 1742, 1673, 1510, 1264, 1050, $697 \mathrm{~cm}^{-1}$; ${ }^{1} \mathbf{H}$ NMR (400 MHz, CDCI3): $\delta, 7.80(\mathrm{~d}, J=$ $8.4 \mathrm{~Hz}, 2 \mathrm{H}), 7.62$ (d, $J=8.4 \mathrm{~Hz}, 2 \mathrm{H}), 7.42$ (d, $J=7.2 \mathrm{~Hz}, 2 \mathrm{H}), 7.40-$

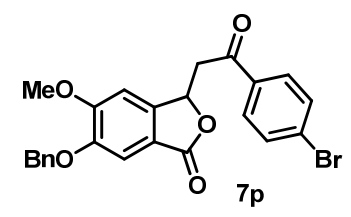
$7.31(\mathrm{~m}, 3 \mathrm{H}), 7.27(\mathrm{~d}, J=22.8 \mathrm{~Hz}, 2 \mathrm{H}), 6.98(\mathrm{~s}, 1 \mathrm{H}), 6.07-5.91(\mathrm{~m}, 1 \mathrm{H}), 5.22-5.11(\mathrm{~m}$, 2H), $3.90(\mathrm{~d}, J=13.7 \mathrm{~Hz}, 3 \mathrm{H}), 3.72(\mathrm{dd}, J=17.7,5.5 \mathrm{~Hz}, 1 \mathrm{H}), 3.28(\mathrm{dd}, J=17.6,7.5 \mathrm{~Hz}$, 1H); ${ }^{13}$ C NMR (101 MHz, $\left.\mathbf{C D C l}_{3}\right): \delta, 195.6,170.2,155.5,149.8,144.4,135.9,134.9,132.2$ (2C), 129.7 (2C), 129.3, 128.7 (2C), 128.3, 127.4 (2C), 117.6, 108.2, 104.6, 76.41, 71.1, 56.4, 43.9; HR-MS (ESI, $\boldsymbol{m} / \mathbf{z})$ : Calculated for $\mathrm{C}_{24} \mathrm{H}_{20} \mathrm{BrO}_{5}\left([\mathrm{M}+\mathrm{H}]^{+}\right): 467.0489$; Found: 467.0496; $[\alpha]_{\mathrm{D}}^{21}=-32.62\left(\mathrm{c}=0.5, \mathrm{CHCl}_{3}, 85 \%\right.$ ee $)$.

The enantiomeric ratio was determined by HPLC analysis using Diacel Chiralpak OD-3 column, $\mathrm{n}$-Hexane/2-Propanol $=65 / 35$, flow rate $=1.0 \mathrm{~mL} / \mathrm{min}, \lambda=254 \mathrm{~nm} ; \mathrm{t}_{\mathrm{R}}=21.0 \mathrm{~min}$ (major), $\mathrm{t}_{\mathrm{R}}=24.8 \mathrm{~min}$ (minor). 
3-(2-(4-Bromophenyl)-2-oxoethyl)-5-methylisobenzofuran-1(3H)-one (7q): (before wash $100 \mathrm{mg} ; 95 \%$ yield; 75\% ee); $81 \mathrm{mg} ; 77 \%$ yield; $\mathrm{R}_{\mathrm{f}}=0.33$ (20:80= EtOAc/n-Hexane); White solid; $\operatorname{mp} 203{ }^{0} \mathrm{C}$; FT-IR (neat): 3020, 2898, 2835, 2357, 2329, 1646, $701 \mathrm{~cm}^{-1}$; ${ }^{\mathbf{1}} \mathbf{H}$ NMR (400 MHz, $\left.\mathbf{C D C l}_{3}\right): \delta, 7.79(\mathrm{dd}, J=12.6,8.2 \mathrm{~Hz}$, $3 \mathrm{H}), 7.63(\mathrm{t}, J=9.9 \mathrm{~Hz}, 2 \mathrm{H}), 7.40-7.28(\mathrm{~m}, 2 \mathrm{H}), 6.08(\mathrm{t}, J=6.4 \mathrm{~Hz}$, $1 \mathrm{H}), 3.68(\mathrm{dd}, J=17.6,5.9 \mathrm{~Hz}, 1 \mathrm{H}), 3.33(\mathrm{dd}, J=17.6,7.0 \mathrm{~Hz}, 1 \mathrm{H})$,

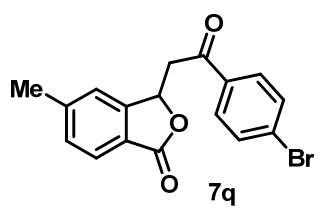

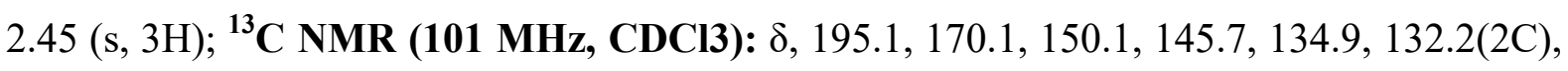
130.7, 129.7(2C), 129.2, 125.6, 123.3, 122.9, 76.7, 43.7, 22.1; HR-MS (ESI, m/z): Calculated for $\mathrm{C}_{17} \mathrm{H}_{14} \mathrm{BrO}_{3}\left([\mathrm{M}+\mathrm{H}]^{+}\right): 345.0121$; Found: $345.0148 ;[\alpha]_{\mathrm{D}}^{22}=-13.72(\mathrm{c}=0.5$, $\mathrm{CHCl}_{3}, 75 \%$ ee).

The enantiomeric ratio was determined by HPLC analysis using Diacel Chiralpak OD-3 column, $n$-Hexane/2-Propanol $=80 / 20$, flow rate $=1.0 \mathrm{~mL} / \mathrm{min}, \lambda=254 \mathrm{~nm} ; \mathrm{t}_{\mathrm{R}}=16.1 \mathrm{~min}$ (major), $\mathrm{t}_{\mathrm{R}}=21.8 \mathrm{~min}$ (minor).

3-(2-(4-Bromophenyl)-2-oxoethyl)-6-chloroisobenzofuran-1(3H)-one (7r): (before wash $98 \mathrm{mg}$; 93\% yield; 91\% ee); $57 \mathrm{mg} ; 54 \%$ yield; $\mathrm{R}_{\mathrm{f}}=0.34$ (20:80 = EtOAc/n-Hexane); White solid; mp $190{ }^{0} \mathrm{C}$; FT-IR (neat): 3020,2877, 2834, 2354, 2336, 1745, 1671, 1503, 1454, 1050, $700 \mathrm{~cm}^{-1}$; ${ }^{1}$ H NMR (400 MHz, CDCI3): $\delta$, $7.88-7.83(\mathrm{~m}, 1 \mathrm{H}), 7.79(\mathrm{~d}, J=8.6 \mathrm{~Hz}, 2 \mathrm{H}), 7.65-7.58(\mathrm{~m}, 3 \mathrm{H})$, $7.51(\mathrm{~d}, J=8.2 \mathrm{~Hz}, 1 \mathrm{H}), 6.10(\mathrm{dd}, J=7.2,5.8 \mathrm{~Hz}, 1 \mathrm{H}), 3.74(\mathrm{dd}, J=$

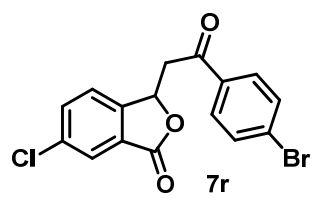
17.8, $5.5 \mathrm{~Hz}, 1 \mathrm{H}), 3.40$ - 3.27 (m, 1H); ${ }^{13} \mathbf{C}$ NMR (101 MHz, CDCl3): $\delta, ~ 194.9,168.6$, 147.7, 135.9, 134.7, 134.6, 132.3 (2C), 129.6 (2C), 129.4, 127.7, 125.7, 124.3, 76.9, 43.5; HR-MS (ESI, $\boldsymbol{m} / \mathbf{z}$ ): Calculated for $\mathrm{C}_{16} \mathrm{H}_{10} \mathrm{BrClNaO}_{3}\left([\mathrm{M}+\mathrm{Na}]^{+}\right)$: 386.9394;Found: 386.9405; $[\alpha]_{\mathrm{D}}^{20}=-41.78\left(\mathrm{c}=0.5, \mathrm{CHCl}_{3}, 98 \%\right.$ ee $)$.

The enantiomeric ratio was determined by HPLC analysis using Diacel Chiralpak OD-3 column, $\mathrm{n}$-Hexane/2-Propanol $=75 / 25$, flow rate $=1.0 \mathrm{~mL} / \mathrm{min}, \lambda=254 \mathrm{~nm} ; \mathrm{t}_{\mathrm{R}}=21.5 \mathrm{~min}$ (major), $\mathrm{t}_{\mathrm{R}}=26.9 \mathrm{~min}$ (minor).

6-Bromo-3-(2-(4-bromophenyl)-2-oxoethyl)isobenzofuran-1(3H)-one (7s): (before wash $88 \mathrm{mg} ; 84 \%$ yield; 90\% ee); $73 \mathrm{mg} ; 70 \%$ yield; $\mathrm{R}_{\mathrm{f}}=0.34$ (20:80 = EtOAc/n-Hexane); Brown solid; mp $171{ }^{0} \mathrm{C}$; FT-IR (neat): 3020, 2870, 2838, 2359, 1660, 1524, 1445, 748, $649 \mathrm{~cm}^{-1}$; ${ }^{1}$ H NMR (400 MHz, CDCl3): $\delta, 8.01(\mathrm{~s}, 1 \mathrm{H}), 7.79(\mathrm{~d}, J=8.6 \mathrm{~Hz}$, 2H), $7.75(\mathrm{dd}, J=8.2,1.7 \mathrm{~Hz}, 1 \mathrm{H}), 7.62(\mathrm{~d}, J=8.5 \mathrm{~Hz}, 2 \mathrm{H}), 7.45(\mathrm{~d}$,

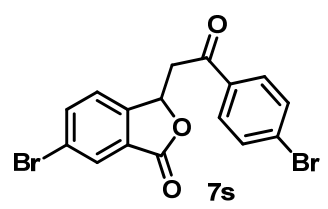


$J=8.2 \mathrm{~Hz}, 1 \mathrm{H}), 6.16-6.02(\mathrm{~m}, 1 \mathrm{H}), 3.74(\mathrm{dd}, J=17.8,5.5 \mathrm{~Hz}, 1 \mathrm{H}), 3.33(\mathrm{dd}, J=17.8,7.5$ $\mathrm{Hz}, 1 \mathrm{H}) ;{ }^{13} \mathbf{C}$ NMR (101 MHz, CDCl3): $\delta, 194.9,168.4,148.2,137.4,134.7,132.3(2 \mathrm{C})$, 129.6(2C), 129.4, 128.8, 128.0, 124.5, 123.6, 76.9, 43.4; HR-MS (ESI, m/z): Calculated for $\mathrm{C}_{16} \mathrm{H}_{10} \mathrm{Br}_{2} \mathrm{NaO}_{3}\left([\mathrm{M}+\mathrm{Na}]^{+}\right): 430.8889$; Found: 430.8900; $[\alpha]_{\mathrm{D}}{ }^{20}=-21.88\left(\mathrm{c}=0.5, \mathrm{CHCl}_{3}\right.$, $97 \%$ ee).

The enantiomeric ratio was determined by HPLC analysis using Diacel Chiralpak OD-3 column, $\mathrm{n}$-Hexane/2-Propanol $=75 / 25$, flow rate $=1.0 \mathrm{~mL} / \mathrm{min}, \lambda=254 \mathrm{~nm} ; \mathrm{t}_{\mathrm{R}}=22.1 \mathrm{~min}$ (major), $\mathrm{t}_{\mathrm{R}}=27.5 \mathrm{~min}$ (minor).

3-(2-(4-Bromophenyl)-2-oxoethyl)-6-fluoroisobenzofuran-1(3H)-one (7t): (before wash $88 \mathrm{mg} ; 84 \%$ yield; $87 \%$ ee $) ; 50 \mathrm{mg} ; 47 \%$ yield; $\mathrm{R}_{\mathrm{f}}=0.26(20: 80=$ EtOAc/n-Hexane); White solid; mp $194{ }^{0} \mathrm{C}$; FT-IR (neat): 3021, 2883, 2830, 2358, 1732, 1658, 1501, 991, $701 \mathrm{~cm}^{-1}$; ${ }^{\mathbf{1}} \mathbf{H}$ NMR (400 MHz, CDC13): $\delta, 7.80(\mathrm{~d}, J=8.5 \mathrm{~Hz}, 2 \mathrm{H}), 7.73-7.58(\mathrm{~m}, 2 \mathrm{H}), 7.58$

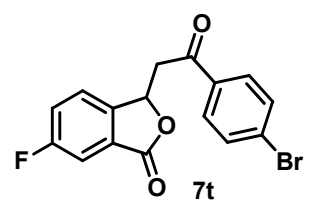
- 7.49 (m, 2H), 7.36 (td, $J=8.6,2.3 \mathrm{~Hz}, 1 \mathrm{H}), 6.10$ (t, $J=6.4 \mathrm{~Hz}, 1 \mathrm{H}), 3.75$ (dd, $J=17.7,5.5$ $\mathrm{Hz}, 1 \mathrm{H}), 3.33$ (dd, $J=17.7,7.6 \mathrm{~Hz}, 1 \mathrm{H}) ;{ }^{13} \mathbf{C}$ NMR (101 MHz, $\left.\mathbf{C D C l}_{3}\right): \delta, 195.0,168.8$ (d, $J$ $=3.8 \mathrm{~Hz}), 163.4(\mathrm{~d}, J=250.3 \mathrm{~Hz}), 145.1(\mathrm{~d}, J=2.3 \mathrm{~Hz}), 134.73,132.26(2 \mathrm{C}), 129.63(2 \mathrm{C})$, 129.39, $128.0(\mathrm{~d}, J=9.1 \mathrm{~Hz}), 124.8(\mathrm{~d}, J=8.5 \mathrm{~Hz}), 122.3(\mathrm{~d}, J=23.8 \mathrm{~Hz}), 112.15$ (d, $J=$ $23.7 \mathrm{~Hz}$ ), 76.96, 43.63; HR-MS (ESI, m/z): Calculated for $\mathrm{C}_{16} \mathrm{H}_{10} \mathrm{BrFNaO}_{3}$ $\left([\mathrm{M}+\mathrm{Na}]^{+}\right): 370.9690$; Found: $370.9704 ;[\alpha]_{\mathrm{D}}{ }^{20}=-67.5\left(\mathrm{c}=0.5, \mathrm{CHCl}_{3},>99 \%\right.$ ee $)$.

The enantiomeric ratio was determined by HPLC analysis using Diacel Chiralpak OD-3 column, $\mathrm{n}$-Hexane/2-Propanol $=75 / 25$, flow rate $=1.0 \mathrm{~mL} / \mathrm{min}, \lambda=254 \mathrm{~nm} ; \mathrm{t}_{\mathrm{R}}=19.6 \mathrm{~min}$ (major), $\mathrm{t}_{\mathrm{R}}=23.7 \mathrm{~min}$ (minor).

3-(2-(4-Bromophenyl)-2-oxoethyl)-7-fluoroisobenzofuran-1(3H)-one (7u): (before wash $98 \mathrm{mg} ; 93 \%$ yield; 89\% ee); $63 \mathrm{mg} ; 60 \%$ yield; $\mathrm{R}_{\mathrm{f}}=0.27$ (20:80 = EtOAc/n-Hexane); White Solid; $183{ }^{0} \mathrm{C}$; FT-IR (neat): 3021, 2981, 2990, 2829, 2358, 1669, 1509, 1003, $698 \mathrm{~cm}^{-1} ;{ }^{1} \mathbf{H}$ NMR (400 MHz, $\left.\mathbf{C D C l}_{3}\right): \delta 7.79(\mathrm{~d}, J=8.6$ $\mathrm{Hz}, 2 \mathrm{H}), 7.61(\mathrm{~d}, J=8.6 \mathrm{~Hz}, 3 \mathrm{H}), 7.32(\mathrm{~d}, J=7.6 \mathrm{~Hz}, 1 \mathrm{H}), 7.15$ (t, $J=$

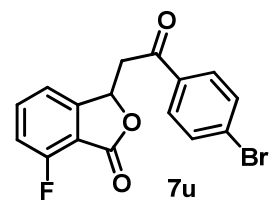
$8.5 \mathrm{~Hz}, 1 \mathrm{H}), 6.11(\mathrm{t}, J=6.4 \mathrm{~Hz}, 1 \mathrm{H}), 3.71(\mathrm{dd}, J=17.7,5.8 \mathrm{~Hz}, 1 \mathrm{H})$, $3.37(\mathrm{dd}, J=17.7,7.0 \mathrm{~Hz}, 1 \mathrm{H}) ;{ }^{13} \mathbf{C}$ NMR (101 $\left.\mathbf{M H z}, \mathbf{C D C l}_{3}\right): \delta 194.76,166.0(\mathrm{~d}, J=2.9$ Hz), 159.7 (d, $J=264.9 \mathrm{~Hz}), 152.1$ (d, $J=1.3 \mathrm{~Hz}), 136.9$ (d, $J=7.8 \mathrm{~Hz}), 134.75,132.24$ (2C), 129.65 (2C), 129.36, $118.7(\mathrm{~d}, J=4.5 \mathrm{~Hz}), 116.5$ (d, $J=18.7 \mathrm{~Hz}), 113.8$ (d, $J=14.3$ 
$\mathrm{Hz}$ ), 76.63, 43.48; HR-MS (ESI, m/z): Calculated for $\mathrm{C}_{16} \mathrm{H}_{11} \mathrm{BrFO}_{3}\left([\mathrm{M}+\mathrm{H}]^{+}\right): 348.9870$; Found: $348.9862 ;[\alpha]_{\mathrm{D}}^{20}=-42.3\left(\mathrm{c}=0.5, \mathrm{CHCl}_{3}, 96 \%\right.$ ee $)$.

The enantiomeric ratio was determined by HPLC analysis using Diacel Chiralpak OD-3 column, $\mathrm{n}$-Hexane/2-Propanol $=65 / 35$, flow rate $=1.0 \mathrm{~mL} / \mathrm{min}, \lambda=254 \mathrm{~nm} ; \mathrm{t}_{\mathrm{R}}=15.1 \mathrm{~min}$ (major), $\mathrm{t}_{\mathrm{R}}=23.2 \mathrm{~min}$ (minor).

7-Fluoro-3-(2-oxo-2-(p-tolyl)ethyl)isobenzofuran-1(3H)-one (7v): (before wash $30 \mathrm{mg}$; 95\% yield; 88\% ee); $17 \mathrm{mg} ; 53 \%$ yield; $\mathrm{R}_{\mathrm{f}}=0.21$ (20:80 = EtOAc/n-Hexane); Brown solid; mp $140{ }^{0} \mathrm{C}$; FT-IR (neat): 2918, 2852, 2360, 1743, 1662, 1493, 1031, 750, $652 \mathrm{~cm}^{-1}$; ${ }^{1}$ H NMR (400 MHz, CDCl3): $\delta, 7.83(\mathrm{~d}, J=$ $8.2 \mathrm{~Hz}, 2 \mathrm{H}), 7.62(\mathrm{td}, J=7.9,4.7 \mathrm{~Hz}, 1 \mathrm{H}), 7.34$ (d, $J=7.7 \mathrm{~Hz}, 1 \mathrm{H})$,

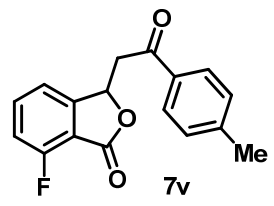
$7.27(\mathrm{~d}, J=8.1 \mathrm{~Hz}, 2 \mathrm{H}), 7.15(\mathrm{t}, J=8.5 \mathrm{~Hz}, 1 \mathrm{H}), 6.14(\mathrm{dd}, J=7.3,5.7 \mathrm{~Hz}, 1 \mathrm{H}), 3.75(\mathrm{dd}, J=$ 17.6, $5.5 \mathrm{~Hz}, 1 \mathrm{H}), 3.36(\mathrm{dd}, J=17.6,7.5 \mathrm{~Hz}, 1 \mathrm{H}), 2.41(\mathrm{~s}, 3 \mathrm{H}) ;{ }^{13} \mathbf{C}$ NMR (101 MHz, $\left.\mathbf{C D C l}_{3}\right) \delta, 195.4,166.2(\mathrm{~d}, J=3.0 \mathrm{~Hz}), 159.6(\mathrm{~d}, J=264.7 \mathrm{~Hz}), 152.4,145.0,136.8(\mathrm{~d}, J=$ $7.7 \mathrm{~Hz}), 133.6,129.6$ (2C), 128.3 (2C), 118.9 (d, $J=4.5 \mathrm{~Hz}), 116.4$ (d, $J=18.7 \mathrm{~Hz}), 113.8$ $(\mathrm{d}, J=14.2 \mathrm{~Hz}), 76.9,43.5,21.7$; HR-MS (ESI, $\mathbf{m} / \mathbf{z})$ : Calculated for $\mathrm{C}_{17} \mathrm{H}_{14} \mathrm{FO}_{3}\left([\mathrm{M}+\mathrm{H}]^{+}\right)$: 285.0921; Found: $285.0921 ;[\alpha]_{\mathrm{D}}^{21}=-52.78\left(\mathrm{c}=0.5, \mathrm{CHCl}_{3}, 92 \%\right.$ ee $)$.

The enantiomeric ratio was determined by HPLC analysis using Diacel Chiralpak OD-3 column, $\mathrm{n}$-Hexane $/ 2$-Propanol $=75 / 25$, flow rate $=1.0 \mathrm{~mL} / \mathrm{min}, \lambda=254 \mathrm{~nm} ; \mathrm{t}_{\mathrm{R}}=13.4 \mathrm{~min}$ (major), $\mathrm{t}_{\mathrm{R}}=18.8 \mathrm{~min}$ (minor).

1-(2-(4-Bromophenyl)-2-oxoethyl)naphtho[1,2-c]furan-3(1H)-one $(\mathbf{7 w})$ : (before wash 98 mg; 93\% yield; 90\% ee); 51 mg; 49\% yield; $\mathrm{R}_{\mathrm{f}}=0.37$ (20:80 = EtOAc/n-Hexane); Light Red Solid; mp $149{ }^{0} \mathrm{C}$; FT-IR (neat): 3075, 3009, 2884, 2836, 2360, 1752, 1678, 1505, 1270, 1067, 760, $650 \mathrm{~cm}^{-1} ;{ }^{1} \mathbf{H}$ NMR (400 MHz, $\left.\mathbf{C D C l}_{3}\right): \delta, 8.04(\mathrm{~d}, J=8.0 \mathrm{~Hz}, 1 \mathrm{H}), 8.01-7.96(\mathrm{~m}, 1 \mathrm{H}), 7.87(\mathrm{~d}, J=$ $7.7 \mathrm{~Hz}, 2 \mathrm{H}), 7.79$ (d, $J=6.1 \mathrm{~Hz}, 2 \mathrm{H}), 7.69$ (d, $J=10.4 \mathrm{~Hz}, 2 \mathrm{H}), 7.61$

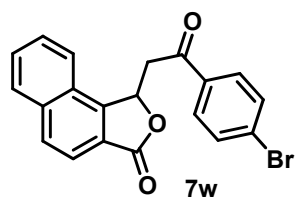
- 7.57 (m, 2H), 6.56 (dd, $J=11.0,8.5 \mathrm{~Hz}, 1 \mathrm{H}), 3.67$ (dd, $J=29.8,12.7 \mathrm{~Hz}, 1 \mathrm{H}), 3.57-3.43$ $(\mathrm{m}, 1 \mathrm{H}) ;{ }^{13} \mathrm{C}$ NMR (101 MHz, CDCl3) $\delta, 194.5,170.4,148.8,136.4,135.0,132.2$ (3C), 130.8, 130.4, 129.8 (2C), 129.7, 129.2, 129.1, 128.2, 126.5, 123.8, 123.5, 120.6, 76.4, 43.8; HR-MS (ESI, m/z): Calculated for $\mathrm{C}_{20} \mathrm{H}_{14} \mathrm{BrO}_{3}\left([\mathrm{M}+\mathrm{H}]^{+}\right)$: 381.0121; Found: 381.0130; $[\alpha]_{\mathrm{D}}^{19}=-7.84\left(\mathrm{c}=0.5, \mathrm{CHCl}_{3}, 95 \%\right.$ ee $)$. 
The enantiomeric ratio was determined by HPLC analysis using Diacel Chiralpak OD-3 column, $\mathrm{n}$-Hexane/2-Propanol $=75 / 25$, flow rate $=1.0 \mathrm{~mL} / \mathrm{min}, \lambda=254 \mathrm{~nm} ; \mathrm{t}_{\mathrm{R}}=33.3 \mathrm{~min}$ (major), $\mathrm{t}_{\mathrm{R}}=21.6 \mathrm{~min}$ (minor).

3-(2-Oxobutyl)isobenzofuran-1(3H)-one (7x): $19 \mathrm{mg}, 58 \%$ yield; $\mathrm{R}_{\mathrm{f}}=0.24(20: 80=$ EtOAc/n-Hexane); White Solid, mp $46{ }^{\circ} \mathrm{C}$; FT-IR (neat): 3075, 3029, 2884, 2865, 1745, 1685, 1500, 1200, 1067, 943, 870, $640 \mathrm{~cm}^{-1} ;{ }^{1} \mathbf{H}$ NMR (400 MHz, $\left.\mathbf{C D C l}_{3}\right): \delta, 7.86(\mathrm{~d}, J=7.6 \mathrm{~Hz}, 1 \mathrm{H}), 7.64(\mathrm{td}, J=7.6,0.7 \mathrm{~Hz}, 1 \mathrm{H}), 7.51(\mathrm{t}$, $J=7.5 \mathrm{~Hz}, 1 \mathrm{H}), 7.44(\mathrm{~d}, J=7.7 \mathrm{~Hz}, 1 \mathrm{H}), 5.92(\mathrm{t}, J=6.6 \mathrm{~Hz}, 1 \mathrm{H}), 3.07$ $(\mathrm{dd}, J=17.2,6.9 \mathrm{~Hz}, 1 \mathrm{H}), 2.86(\mathrm{dd}, J=17.2,6.2 \mathrm{~Hz}, 1 \mathrm{H}), 2.50(\mathrm{dq}, J=$

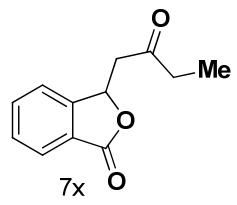
12.5, 7.3 Hz, 2H), 1.08 (t, $J=7.3 \mathrm{~Hz}, 3 \mathrm{H}) ;{ }^{13} \mathbf{C}$ NMR (101 MHz, $\left.\mathbf{C D C l}_{3}\right): \delta, 207.3,170.1$, 149.5, 134.3, 129.4, 125.8, 125.8, 122.3, 76.9, 46.9, 36.8, 7.5; LR-MS (ESI, m/z): Calculated for $\mathrm{C}_{12} \mathrm{H}_{12} \mathrm{O}_{3}\left([\mathrm{M}]^{+}\right)$: 204.2219; Found: 204.2; $[\alpha]_{\mathrm{D}}{ }^{24}=-1.209$ (c=0.910, $\mathrm{CHCl}_{3}, 98 \%$ ee).

The enantiomeric ratio was determined by HPLC analysis using Diacel Chiralpak OD-3 column, $\mathrm{n}$-Hexane $/ 2$-Propanol $=75 / 25$, flow rate $=1.0 \mathrm{~mL} / \mathrm{min}, \lambda=254 \mathrm{~nm} ; \mathrm{t}_{\mathrm{R}}=8.2 \mathrm{~min}$ (major), $\mathrm{t}_{\mathrm{R}}=10.4 \mathrm{~min}$ (minor).

Synthetic Procedure for (E)-6-Hydroxy-1-phenylhex-2-en-1-one/ (E)-6-Hydroxy-1-(ptolyl)hex-2-en-1-one, see: ref. 4

For experimental data of 8d-e, see: ref. 3

Synthetic Procedure for (E)-6-Oxo-6-phenylhex-4-enal/ (E)-6-Oxo-6-(p-tolyl)hex-4-enal: (E)-6-hydroxy-1-phenylhex-2-en-1-one/ (E)-6-hydroxy-1-(p-tolyl)hex-2-en-1-one (1 equiv, $0.5 \mathrm{mmol}$ ) was taken in a round bottom flask with a bar magnet and was placed over a magnetic stirrer. 1.5 equiv of solid DMP was added to the reaction flask portion-wise. The reaction was allowed to run at room temperature for $1 \mathrm{~h}$ to $1.5 \mathrm{~h}$ until all the reactants were consumed. The reaction was quenched with saturated sodium thiosulfate solution and then extracted with $3 \times 10 \mathrm{ml}$ dichloromethane. Then anhydrous $\mathrm{MgSO}_{4}$ was added to the organic extract and was filtered to a round bottom flask. The solvent was removed in vaccuo. The column chromatography was carried out with $10 \%$ EtOAc in n-Hexane to give colourless oil.

(E)-6-Oxo-6-(p-tolyl)hex-4-enal (8a): $60 \mathrm{mg} ; 52 \%$ yield; $\mathrm{R}_{\mathrm{f}}=0.18(20: 80=$ EtOAc/nHexane); Colourless oil; FT-IR (neat): 3024, 2987, 2970, 2856, 1670,

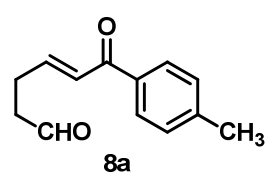


1526, 1498, 1210, 1025, $780 \mathrm{~cm}^{-1}$; ${ }^{1} \mathbf{H}$ NMR (400 MHz, $\left.\mathbf{C D C l}_{3}\right): \delta, 9.81$ (s, 1H), 7.81 (d, $J=$ $8.2 \mathrm{~Hz}, 2 \mathrm{H}), 7.28-7.22(\mathrm{~m}, 2 \mathrm{H}), 6.97(\mathrm{ddd}, J=32.9,19.6,10.8 \mathrm{~Hz}, 2 \mathrm{H}), 3.15-2.42(\mathrm{~m}$, 4H), 2.40 (s, 3H); ${ }^{13} \mathbf{C}$ NMR (101 MHz, $\left.\mathbf{C D C l}_{3}\right): \delta, 200.5,189.9,146.1,143.7,135.1,129.3$ (2C), 128.7 (2C), 126.8, 42.1, 25.0, 21.7; HR-MS (ESI, m/z): Calculated for $\mathrm{C}_{13} \mathrm{H}_{15} \mathrm{O}_{4}$ $\left([\mathrm{M}+\mathrm{H}]^{+}\right): 203.1067$; Found: 203.1085.

(E)-6-(4-Methoxyphenyl)-6-oxohex-4-enal (8b): $102 \mathrm{mg}, 72 \%$ yield; $\mathrm{R}_{\mathrm{f}}=0.12$ (25:75 = EtOAc/n-Hexane); Light yellow sticky liquid; FT-IR (neat): 2980, 2844, 2810, 1670, 1460, 1050, $740 \mathrm{~cm}^{-1} ;{ }^{1} \mathbf{H}$ NMR (400 MHz, $\left.\mathbf{C D C l}_{3}\right): \delta, 9.78(\mathrm{~s}, 1 \mathrm{H}), 8.12-$ $7.74(\mathrm{~m}, 2 \mathrm{H}), 7.16-6.54(\mathrm{~m}, 4 \mathrm{H}), 3.83(\mathrm{~s}, 4 \mathrm{H}), 2.66(\mathrm{~d}, J=6.3 \mathrm{~Hz}, 2 \mathrm{H})$, $2.61(\mathrm{t}, J=6.0 \mathrm{~Hz}, 2 \mathrm{H}) ;{ }^{13} \mathbf{C}$ NMR (101 MHz, $\left.\mathbf{C D C l}_{3}\right): \delta, 200.6,188.6$,

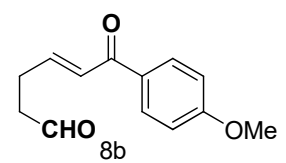
163.5, 145.6, 130.8 (2C), 130.5, 126.5, 113.8 (2C), 55.5, 42.1, 24.9; HRMS (ESI, m/z): Calculated for $\mathrm{C}_{13} \mathrm{H}_{15} \mathrm{O}_{3}\left([\mathrm{M}+\mathrm{H}]^{+}\right)$: 219.1016 ; Found: 219.1042 .

(E)-6-(Furan-2-yl)-6-oxohex-4-enal (8e): 86 mg, 76\% yield; $\mathrm{R}_{\mathrm{f}}=0.15(20: 80=\mathrm{EtOAc} / \mathrm{n}$ Hexane); Yellow sticky liquid; FT-IR (neat): 2980, 2866, 2840, 1666, 1460, $765 \mathrm{~cm}^{-1} ;{ }^{1} \mathbf{H}$ NMR (400 MHz, $\left.\mathbf{C D C l}_{3}\right): \delta, 9.80$ (s, 1H), 7.60 (d, $J=0.9 \mathrm{~Hz}$, 1H), $7.23(\mathrm{~d}, J=3.5 \mathrm{~Hz}, 1 \mathrm{H}), 7.08(\mathrm{dt}, J=15.4,6.5 \mathrm{~Hz}, 1 \mathrm{H}), 6.82$ (dd, $J=$

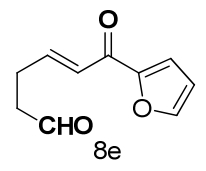
15.5, $1.4 \mathrm{~Hz}, 1 \mathrm{H}), 6.54(\mathrm{dd}, J=3.5,1.7 \mathrm{~Hz}, 1 \mathrm{H}), 2.72-2.65(\mathrm{~m}, 2 \mathrm{H}), 2.62(\mathrm{t}, J=6.7 \mathrm{~Hz}$, 2H); ${ }^{13} \mathbf{C}$ NMR (101 MHz, $\left.\mathbf{C D C l}_{3}\right): \delta, 200.4,177.8,153.2,146.7,146.0,125.8,117.8,112.5$, 41.9, 24.9; HR-MS (ESI, m/z): Calculated for $\mathrm{C}_{10} \mathrm{H}_{11} \mathrm{O}_{3}\left([\mathrm{M}+\mathrm{H}]^{+}\right)$: 179.0703; Found: 179.0738 .

(E)-6-Oxo-6-(thiophen-2-yl)hex-4-enal (8f): $44 \mathrm{mg}, 55 \%$ yield; $\mathrm{R}_{\mathrm{f}}=0.19(20: 80=$ EtOAc/n-Hexane); White Solid; mp $43{ }^{\circ} \mathrm{C}$; FT-IR (neat): 2986, 2836, 2800, 1566, 1470, $755 \mathrm{~cm}^{-1} ;{ }^{1} \mathbf{H}$ NMR (400 MHz, $\mathbf{C D C l}_{3}$ ): $\delta, 9.77$ (s, 1H), 7.71 (dd, $J$ $=3.8,0.8 \mathrm{~Hz}, 1 \mathrm{H}), 7.62(\mathrm{dd}, J=4.9,0.9 \mathrm{~Hz}, 1 \mathrm{H}), 7.10(\mathrm{dd}, J=4.8,3.9 \mathrm{~Hz}, 1 \mathrm{H})$,

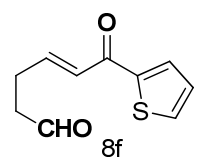
$7.07-6.94(\mathrm{~m}, 1 \mathrm{H}), 6.80$ (d, $J=15.3 \mathrm{~Hz}, 1 \mathrm{H}), 2.69-2.63$ (m, 2H), 2.60 (dd, J = 9.6, $3.9 \mathrm{~Hz}$, 2H); ${ }^{13}$ C NMR (101 MHz, $\left.\mathbf{C D C l}_{3}\right): \delta, 200.5,181.9,146.0,144.9,134.1,132.1,128.3,126.3$, 41.9, 24.9; HR-MS (ESI, m/z): Calculated for $\mathrm{C}_{10} \mathrm{H}_{11} \mathrm{O}_{2} \mathrm{~S}\left([\mathrm{M}+\mathrm{H}]^{+}\right)$: 195.0474; Found: 195.0471 .

(E)-6-Oxohept-4-enal (8g): $45 \mathrm{mg}, 75 \%$ yield; $\mathrm{R}_{\mathrm{f}}=0.25$ (30:70 = EtOAc/n-Hexane); Colourless liquid; FT-IR (neat): 2977, 2888, 2366, 1625, 977, 833, $620 \mathrm{~cm}^{-1} ;{ }^{1} \mathbf{H}$ NMR (400 MHz, $\left.\mathbf{C D C l}_{3}\right) \delta, 9.78(\mathrm{~s}, 1 \mathrm{H}), 6.76(\mathrm{dt}, J=$

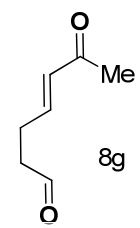


15.9, 6.6 Hz, 1H), $6.07(\mathrm{~d}, J=16.0 \mathrm{~Hz}, 1 \mathrm{H}), 2.64(\mathrm{t}, J=7.1 \mathrm{~Hz}, 2 \mathrm{H}), 2.53(\mathrm{t}, J=6.9 \mathrm{~Hz}, 2 \mathrm{H})$, 2.21 (s, 3H); ${ }^{13} \mathbf{C}$ NMR (101 MHz, $\left.\mathbf{C D C l}_{3}\right): \delta, 200.3,198.3,145.4,131.9,41.9,27.0,24.6$; HR-MS (ESI, $\boldsymbol{m} / \mathbf{z})$ : Calculated for $\mathrm{C}_{7} \mathrm{H}_{11} \mathrm{O}_{2}\left([\mathrm{M}+\mathrm{H}]^{+}\right)$: 127.0754; Found: 127.0779.

\section{Representative synthetic procedure for $\gamma$-lactones:}

\section{Step-I}

A solution of unsaturated enal 8 ( 1 equiv, $0.1 \mathrm{mmol})$ and catalyst $(5 \mathrm{~mol} \%)$ in toluene or diethylether was taken in a small round bottom flask with a magnetic bar and was placed over a magnetic stirrer at room temperature. $6 \mathrm{M}$ solution of TBHP (5 equiv) was added dropwise to the pre-mix solution. The reaction mixture was allowed to stir at room temperature for 12$15 \mathrm{~h}$. The reaction was stopped, when all the starting material were consumed (monitored by TLC). Finally, the reaction was filtered with a small plug of silica and washed with dichloromethane as solvent. The peroxy-acetal was directly taken for step-II after removing the solvent with vaccuo.

\section{Step-II}

The solution of peroxy-acetal (obtained from step-I) in dichloromethane was taken in a small round bottom flask with a bar magnet and was placed over a magnetic stirrer. Solid $\mathrm{FeCl}_{3} \cdot 6 \mathrm{H}_{2} \mathrm{O}(25 \mathrm{~mol} \%)$ was directly added to the flask and the reaction was allowed to stir at room temperature for overnight. Then the reaction was filtered with a small plug of silica gel and washed with dichloromethane as solvent. After the removal of solvent in vaccuo the crude $\gamma$-lactone was washed with n-hexane to get optically pure $\gamma$-lactone.

5-(2-Oxo-2-(p-tolyl)ethyl)dihydrofuran-2(3H)-one (9a): (before wash $31 \mathrm{mg}$; 95\% yield; 96\% ee); 20 mg; 61\% yield; $\mathrm{R}_{\mathrm{f}}=0.25$ (20:80 = EtOAc/n-Hexane); White Solid; mp $92{ }^{0} \mathrm{C}$; FT-IR (neat): 3029, 2936, 2852, 1769, 1677, 1452, 1173, 1029, $809 \mathrm{~cm}^{-}$ ${ }^{1} ;{ }^{1} \mathbf{H}$ NMR (400 MHz, $\left.\mathbf{C D C l}_{3}\right) \delta, 7.82(\mathrm{~d}, J=8.2 \mathrm{~Hz}, 2 \mathrm{H}), 7.26(\mathrm{~d}, J=$ $8.1 \mathrm{~Hz}, 2 \mathrm{H}), 5.15-5.02(\mathrm{~m}, 1 \mathrm{H}), 3.58(\mathrm{dd}, J=17.3,5.2 \mathrm{~Hz}, 1 \mathrm{H}), 3.18$ $(\mathrm{dd}, J=17.3,7.7 \mathrm{~Hz}, 1 \mathrm{H}), 2.66-2.53(\mathrm{~m}, 3 \mathrm{H}), 2.40(\mathrm{~s}, 3 \mathrm{H}), 2.03-1.87$

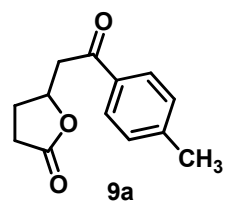
$(\mathrm{m}, 1 \mathrm{H}) ;{ }^{13} \mathbf{C}$ NMR (101 MHz, $\left.\mathbf{C D C l}_{3}\right) \delta, 195.9,176.7,144.7,133.9,129.5$ (2C), 128.2 (2C), 76.8, 43.7, 28.6, 28.3, 21.7; HR-MS (ESI, m/z): Calculated for $\mathrm{C}_{13} \mathrm{H}_{15} \mathrm{O}_{3}\left([\mathrm{M}+\mathrm{H}]^{+}\right)$: 219.1016; Found: 219.0995; $[\alpha]_{\mathrm{D}}{ }^{21}=-4.075\left(\mathrm{c}=0.5, \mathrm{CHCl}_{3}, 99 \%\right.$ ee $)$. 
The enantiomeric ratio was determined by HPLC analysis using Diacel Chiralpak OD-3 column, $\mathrm{n}$-Hexane/2-Propanol $=75 / 25$, flow rate $=1.0 \mathrm{~mL} / \mathrm{min}, \lambda=254 \mathrm{~nm} ; \mathrm{t}_{\mathrm{R}}=12.8 \mathrm{~min}$ (major), $\mathrm{t}_{\mathrm{R}}=15.0 \mathrm{~min}$ (minor).

5-(2-(4-Methoxyphenyl)-2-oxoethyl)dihydrofuran-2(3H)-one (9b): Without wash $20 \mathrm{mg}$, $87 \%$ yield; $\mathrm{R}_{\mathrm{f}}=0.25\left(30: 70=\right.$ EtOAc/n-Hexane); White solid; mp $67{ }^{\circ} \mathrm{C}$; FT-IR (neat): $3040,2988,2858,2366,1760,1685,1533,1255,1041,751,647 \mathrm{~cm}^{-1} ;{ }^{1} \mathbf{H}$ NMR (400 MHz, CDCl 3 ): $\delta, 7.90(\mathrm{~d}, J=8.8 \mathrm{~Hz}, 2 \mathrm{H}), 6.93(\mathrm{~d}, J=8.8 \mathrm{~Hz}$, $2 \mathrm{H}), 5.16-4.95(\mathrm{~m}, 1 \mathrm{H}), 3.85(\mathrm{~s}, 3 \mathrm{H}), 3.55(\mathrm{dd}, J=17.1,5.2 \mathrm{~Hz}, 1 \mathrm{H})$, $3.15(\mathrm{dd}, J=17.1,7.7 \mathrm{~Hz}, 1 \mathrm{H}), 2.76-2.39$ (m, 3H), 1.95 (ddd, $J=12.7$,

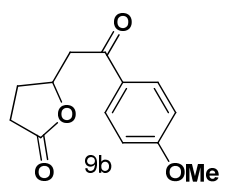
8.2, $5.5 \mathrm{~Hz}, 1 \mathrm{H}) ;{ }^{13} \mathbf{C}$ NMR (101 $\left.\mathbf{M H z}, \mathbf{C D C l}_{3}\right): \delta, 194.8,176.8,163.9,130.4$ (2C), 129.5, 113.9 (2C), 76.9, 55.6, 43.5, 28.6, 28.4; HR-MS (ESI, m/z): Calculated for $\mathrm{C}_{13} \mathrm{H}_{15} \mathrm{O}_{4}$ $\left([\mathrm{M}+\mathrm{H}]^{+}\right): 235.0965$; Found: $235.0986 ;[\alpha]_{\mathrm{D}}{ }^{24}=-2.028\left(\mathrm{c}=0.810, \mathrm{CHCl}_{3}, 92 \%\right.$ ee $)$.

The enantiomeric ratio was determined by HPLC analysis using Diacel Chiralpak OZ-3 column, $\mathrm{n}$-Hexane/2-Propanol $=70 / 30$, flow rate $=1.0 \mathrm{~mL} / \mathrm{min}, \lambda=254 \mathrm{~nm} ; \mathrm{t}_{\mathrm{R}}=43.9 \mathrm{~min}$ (major), $\mathrm{t}_{\mathrm{R}}=41.5 \mathrm{~min}$ (minor).

5-(2-(4-Bromophenyl)-2-oxoethyl)dihydrofuran-2(3H)-one (9c): Without wash $23 \mathrm{mg}$, $82 \%$ yield; $\mathrm{R}_{\mathrm{f}}=0.21\left(20: 80=\right.$ EtOAc/n-Hexane); Brown solid; mp $84{ }^{\circ} \mathrm{C}$; FT-IR (neat): 3050, 2960, 2857, 2361, 1769, 1684, 1265, 1031, 744, 650 $\mathrm{cm}^{-1} ;{ }^{1} \mathbf{H}$ NMR (400 MHz, $\left.\mathbf{C D C l}_{3}\right): \delta, 7.78(\mathrm{~d}, J=8.5 \mathrm{~Hz}, 2 \mathrm{H}), 7.61(\mathrm{~d}, J$ $=8.5 \mathrm{~Hz}, 2 \mathrm{H}), 5.23-4.89(\mathrm{~m}, 1 \mathrm{H}), 3.55(\mathrm{dd}, J=17.4,5.4 \mathrm{~Hz}, 1 \mathrm{H}), 3.17$

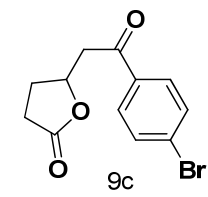
$(\mathrm{dd}, J=17.4,7.2 \mathrm{~Hz}, 1 \mathrm{H}), 2.77-2.45(\mathrm{~m}, 3 \mathrm{H}), 2.07-1.83(\mathrm{~m}, 1 \mathrm{H}) ;{ }^{13} \mathbf{C}$ NMR (101 MHz, $\left.\mathbf{C D C l}_{3}\right): \delta, 195.3,176.6,135.1,132.2$ (2C), 129.6 (2C), 129.0, 76.4, 43.8, 28.6, 28.3; HRMS (ESI, $\boldsymbol{m} / \mathbf{z})$ : Calculated for $\mathrm{C}_{12} \mathrm{H}_{12} \mathrm{BrO}_{3}\left([\mathrm{M}+\mathrm{H}]^{+}\right): 282.9964$; Found: $282.9944 ;[\alpha]_{\mathrm{D}}{ }^{24}=$ $2.117\left(\mathrm{c}=0.695, \mathrm{CHCl}_{3}, 96 \%\right.$ ee $)$.

The enantiomeric ratio was determined by HPLC analysis using Diacel Chiralpak OD-3 column, $\mathrm{n}$-Hexane/2-Propanol $=75 / 25$, flow rate $=1.0 \mathrm{~mL} / \mathrm{min}, \lambda=254 \mathrm{~nm} ; \mathrm{t}_{\mathrm{R}}=20.9 \mathrm{~min}$ (major), $\mathrm{t}_{\mathrm{R}}=27.2 \mathrm{~min}$ (minor).

5-(2-(4-Iodophenyl)-2-oxoethyl)dihydrofuran-2(3H)-one (9d): Without wash $23 \mathrm{mg}, 90 \%$ yield; $\mathrm{R}_{\mathrm{f}}=0.20$ (20:80 = EtOAc/n-Hexane); White solid; mp $109{ }^{\circ} \mathrm{C}$; FT-IR (neat): 3050, 2965, 2855, 2364, 1685, 1031, 755, $654 \mathrm{~cm}^{-1}$; ${ }^{1} \mathbf{H}$ NMR (400 MHz, $\left.\mathbf{C D C l}_{3}\right): \delta, 7.83(\mathrm{~d}, J=7.9 \mathrm{~Hz}, 2 \mathrm{H}), 7.62(\mathrm{~d}, J=8.0 \mathrm{~Hz}, 2 \mathrm{H}), 5.15-4.96$

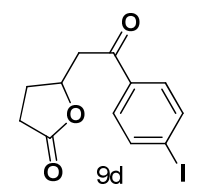


$(\mathrm{m}, 1 \mathrm{H}), 3.54(\mathrm{dd}, J=17.4,5.1 \mathrm{~Hz}, 1 \mathrm{H}), 3.16(\mathrm{dd}, J=17.4,7.2 \mathrm{~Hz}, 1 \mathrm{H}), 2.71-2.46(\mathrm{~m}, 3 \mathrm{H})$, 2.05 - $1.84(\mathrm{~m}, 1 \mathrm{H}) ;{ }^{13} \mathbf{C}$ NMR (101 $\left.\mathbf{M H z}, \mathbf{C D C l} \mathbf{3}\right): \delta, 195.6,176.6,138.2,135.6,129.4$, 101.9, 76.4, 43.7, 28.6, 28.3; HR-MS (ESI, m/z): Calculated for $\mathrm{C}_{12} \mathrm{H}_{12} \mathrm{IO}_{3}\left([\mathrm{M}+\mathrm{H}]^{+}\right)$: 330.9826; Found: $330.9829 ;[\alpha]_{\mathrm{D}}{ }^{24}=1.863\left(\mathrm{c}=1.150, \mathrm{CHCl}_{3}, 95 \%\right.$ ee $)$.

The enantiomeric ratio was determined by HPLC analysis using Diacel Chiralpak OD-3 column, $\mathrm{n}$-Hexane/2-Propanol $=75 / 25$, flow rate $=1.0 \mathrm{~mL} / \mathrm{min}, \lambda=254 \mathrm{~nm} ; \mathrm{t}_{\mathrm{R}}=24.8 \mathrm{~min}$ (major), $\mathrm{t}_{\mathrm{R}}=31.2 \mathrm{~min}$ (minor).

5-(2-(Furan-2-yl)-2-oxoethyl)dihydrofuran-2(3H)-one (9e): (before wash $17 \mathrm{mg}, 87 \%$ yield; 96\% ee); 14 mg, 80\% yield; $\mathrm{R}_{\mathrm{f}}=0.20$ (30:70 = EtOAc/n-Hexane); Grey Soild; mp 102 ${ }^{\circ} \mathrm{C}$; FT-IR (neat): 3044, 2998, 2858, 2366, 1760, 1682, 1255, 1061, 741, $667 \mathrm{~cm}^{-1} ;{ }^{1} \mathbf{H}$ NMR (400 MHz, $\left.\mathbf{C D C l}_{3}\right): \delta, 7.59(\mathrm{~d}, J=0.9 \mathrm{~Hz}, 1 \mathrm{H})$, $7.22(\mathrm{~d}, J=3.4 \mathrm{~Hz}, 1 \mathrm{H}), 6.55(\mathrm{dd}, J=3.5,1.6 \mathrm{~Hz}, 1 \mathrm{H}), 5.04(\mathrm{dd}, J=$ $10.1,3.8 \mathrm{~Hz}, 1 \mathrm{H}), 3.43(\mathrm{dd}, J=16.7,6.0 \mathrm{~Hz}, 1 \mathrm{H}), 3.06(\mathrm{dd}, J=16.7,7.0$

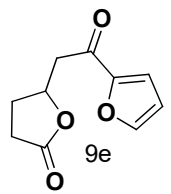
$\mathrm{Hz}, 1 \mathrm{H}), 2.63-2.44(\mathrm{~m}, 3 \mathrm{H}), 2.05-1.88(\mathrm{~m}, 1 \mathrm{H}) ;{ }^{13} \mathbf{C}$ NMR (101 MHz, $\left.\mathbf{C D C l}_{3}\right): \delta, 185.0$, 176.6, 152.4, 146.9, 117.9, 112.6, 76.2, 43.6, 28.5, 28.1; HR-MS (ESI, m/z): Calculated for $\mathrm{C}_{10} \mathrm{H}_{11} \mathrm{O}_{4}\left([\mathrm{M}+\mathrm{H}]^{+}\right)$: 195.0652 ; Found: 195.0679; $[\alpha]_{\mathrm{D}}{ }^{24}=7.571\left(\mathrm{c}=0.300, \mathrm{CHCl}_{3}, 96 \%\right.$ ee $)$.

The enantiomeric ratio was determined by HPLC analysis using Diacel Chiralpak IC-3 column, $\mathrm{n}$-Hexane/2-Propanol $=30 / 70$, flow rate $=1.0 \mathrm{~mL} / \mathrm{min}, \lambda=254 \mathrm{~nm} ; \mathrm{t}_{\mathrm{R}}=36.5 \mathrm{~min}$ (major), $\mathrm{t}_{\mathrm{R}}=27.1 \mathrm{~min}$ (minor).

5-(2-Oxo-2-(thiophen-2-yl)ethyl)dihydrofuran-2(3H)-one (9f): Without wash $18 \mathrm{mg}, 86 \%$ yield; $\mathrm{R}_{\mathrm{f}}=0.25$ (30:70 = EtOAc/n-Hexane); Colourless semisolid; FT-IR (neat): 3103, 3002, 2878, 2396, 1765, 1645, 1066, 751, $647 \mathrm{~cm}^{-1} ;{ }^{1} \mathbf{H}$ NMR (400 MHz, CDCl $\left.\mathbf{3}\right) \delta, 7.79-7.54$ (m, 2H), $7.13(\mathrm{dd}, J=4.7,4.0 \mathrm{~Hz}, 1 \mathrm{H}), 5.17-4.90$ (m, 1H), 3.50 (dd, $J$ $=16.6,5.7 \mathrm{~Hz}, 1 \mathrm{H}), 3.14(\mathrm{dd}, J=16.6,7.1 \mathrm{~Hz}, 1 \mathrm{H}), 2.56(\mathrm{dd}, J=9.2$, $3.7 \mathrm{~Hz}, 3 \mathrm{H}), 2.07-1.87(\mathrm{~m}, 1 \mathrm{H}) ;{ }^{13} \mathbf{C}$ NMR (101 MHz, $\left.\mathbf{C D C l}_{3}\right): \delta$, 188.9, 176.6, 143.7, 134.6, 132.7, 128.4, 76.5, 44.4, 28.6, 28.1; HR-MS

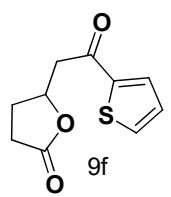
(ESI, $\boldsymbol{m} / \mathbf{z})$ : Calculated for $\mathrm{C}_{10} \mathrm{H}_{11} \mathrm{O}_{3} \mathrm{~S}\left([\mathrm{M}+\mathrm{H}]^{+}\right): 211.0423$; Found: $211.0430 ;[\alpha]_{\mathrm{D}}{ }^{23}=-2.076$ $\left(\mathrm{c}=0.970, \mathrm{CHCl}_{3}, 97 \%\right.$ ee $)$.

The enantiomeric ratio was determined by HPLC analysis using Diacel Chiralpak OZ-3 column, $\mathrm{n}$-Hexane $/ 2$-Propanol $=25 / 75$, flow rate $=1.0 \mathrm{~mL} / \mathrm{min}, \lambda=254 \mathrm{~nm} ; \mathrm{t}_{\mathrm{R}}=37.2 \mathrm{~min}$ (major), $\mathrm{t}_{\mathrm{R}}=32.4 \min$ (minor). 
5-(2-Oxopropyl)dihydrofuran-2(3H)-one (9g): Without wash $16 \mathrm{mg}, 56 \%$ yield; $\mathrm{R}_{\mathrm{f}}=0.15$

(30:70 = EtOAc/n-Hexane); Colourless liquid; FT-IR (neat): 2366, 1673, 1519, 977, 843, 821, 750, $657 \mathrm{~cm}^{-1} ;{ }^{1} \mathbf{H}$ NMR (400 MHz, $\left.\mathbf{C D C l}_{3}\right): \delta, 4.86$ (tt, $J=12.5,6.3 \mathrm{~Hz}, 1 \mathrm{H}), 2.97(\mathrm{dd}, J=17.3,6.3 \mathrm{~Hz}, 1 \mathrm{H}), 2.69$ (dd, $J=$ 17.3, 6.4 Hz, 1H), $2.60-2.39$ (m, 3H), 2.18 (s, 3H), $1.95-1.74(\mathrm{~m}, 1 \mathrm{H})$; ${ }^{13}$ C NMR (101 MHz, $\left.\mathbf{C D C l}_{3}\right): \delta, 204.8,176.6,76.1,48.5,30.7,28.5,28.0$;

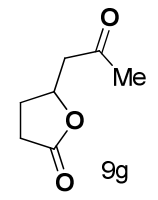

HR-MS (ESI, $\boldsymbol{m} / \mathbf{z})$ : Calculated for $\mathrm{C}_{7} \mathrm{H}_{10} \mathrm{O}_{3} \mathrm{Na}\left([\mathrm{M}+\mathrm{Na}]^{+}\right)$: 165.0522; Found: 165.0546; $[\alpha]_{\mathrm{D}}^{23}=12.774\left(\mathrm{c}=0.775, \mathrm{CHCl}_{3}, 90 \%\right.$ ee $)$.

The enantiomeric ratio was determined by HPLC analysis using Diacel Chiralpak OZ-3 column, $\mathrm{n}$-Hexane $/ 2$-Propanol $=25 / 75$, flow rate $=1.0 \mathrm{~mL} / \mathrm{min}, \lambda=300 \mathrm{~nm} ; \mathrm{t}_{\mathrm{R}}=25.4 \mathrm{~min}$ (major), $\mathrm{t}_{\mathrm{R}}=20.6 \mathrm{~min}$ (minor).

\section{Reaction of in situ generated ortho-carboxylic acid containing chalcone:}

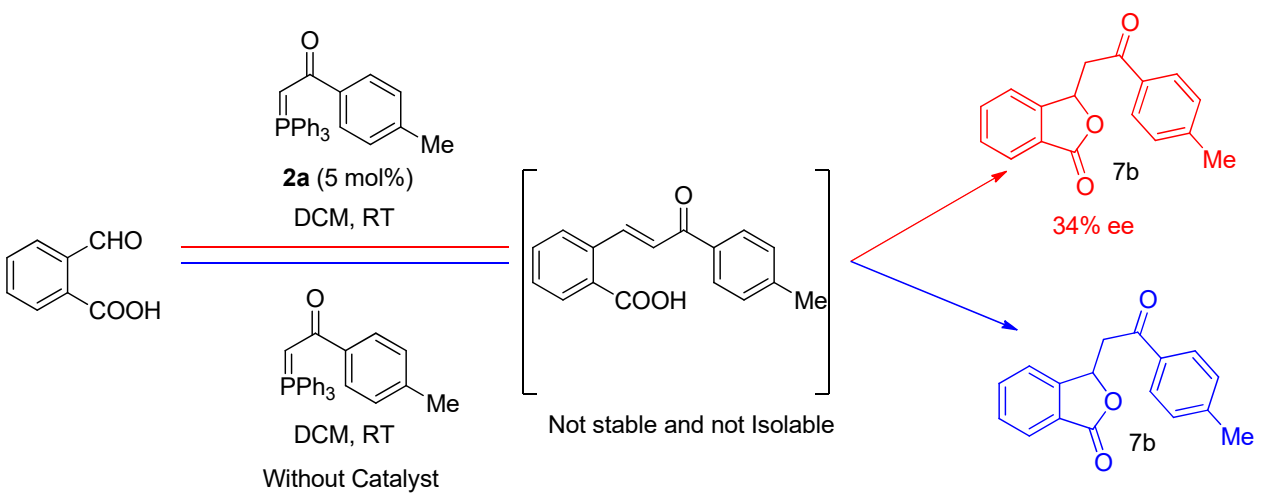

Racemic

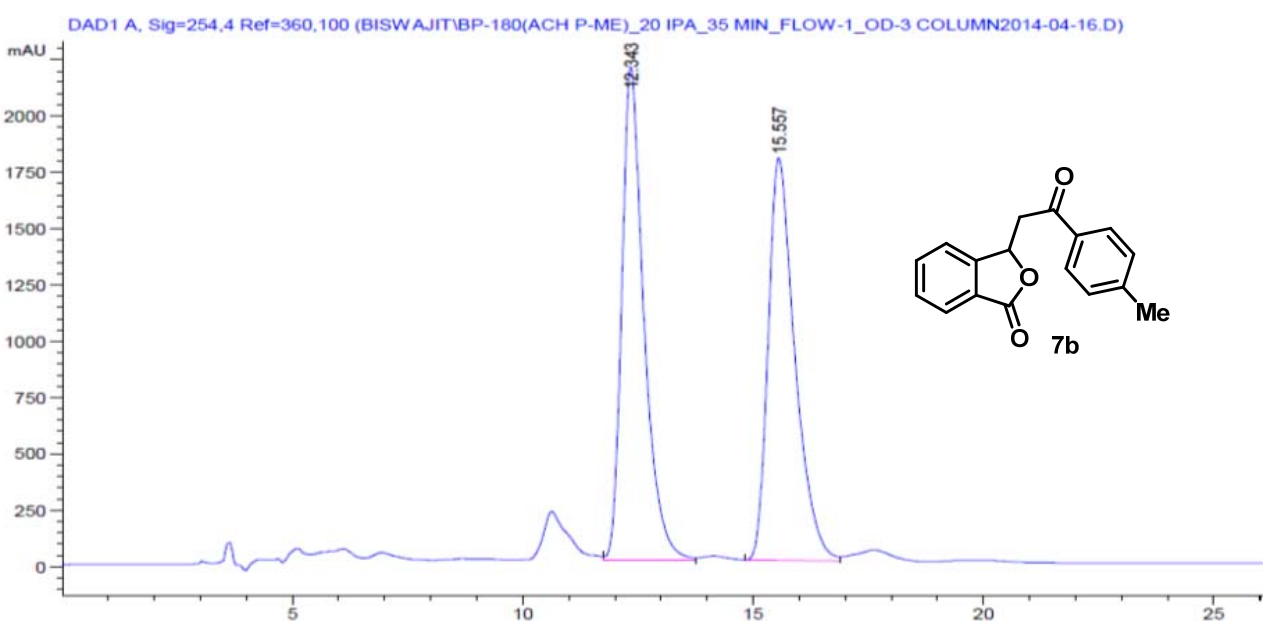




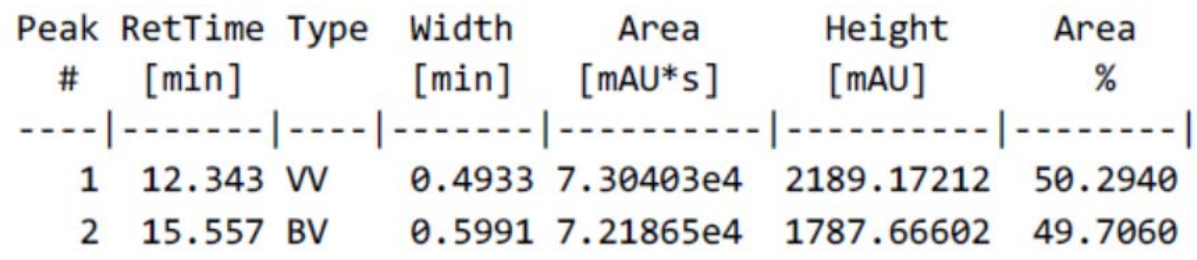

Totals : $\quad 1.45227 \mathrm{e} 5 \quad 3976.83813$

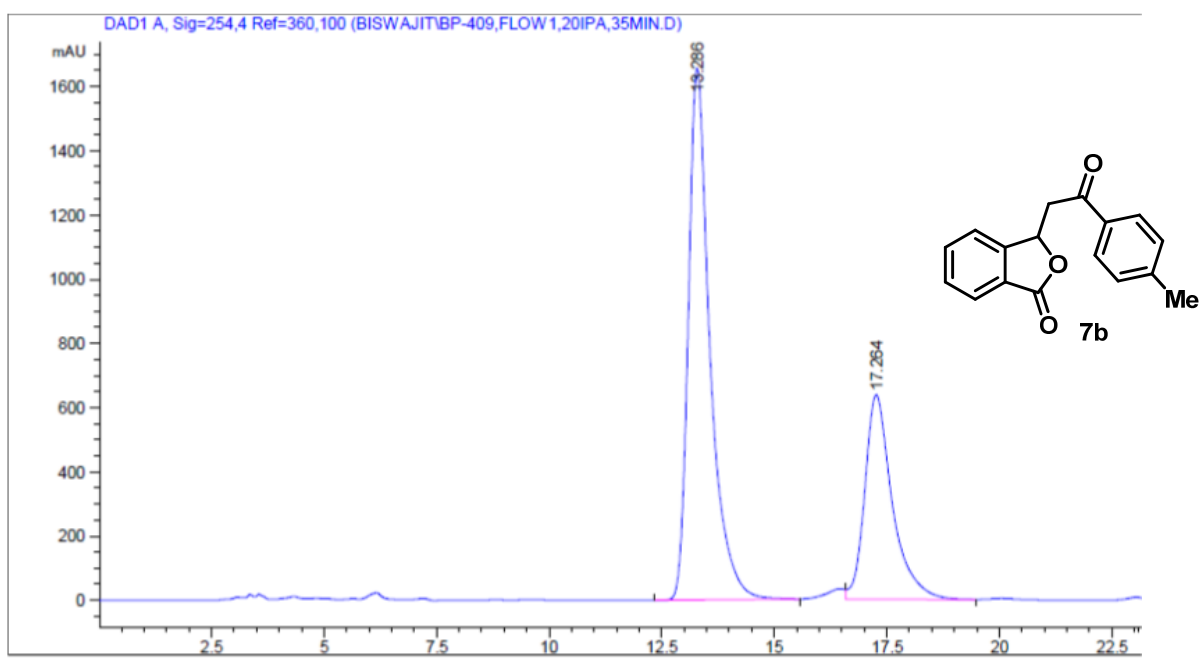

Signal 1: DAD1 A, Sig $=254,4$ Ref $=360,100$

\begin{tabular}{|c|c|c|c|c|c|c|}
\hline $\begin{array}{c}\text { Peak } \\
\#\end{array}$ & $\begin{array}{l}\text { RetTime } \\
\text { [min] }\end{array}$ & Type & $\begin{array}{l}\text { Width } \\
\text { [min] }\end{array}$ & $\begin{array}{c}\text { Area } \\
{\left[\mathrm{mAU}^{*} \mathrm{~s}\right]}\end{array}$ & $\begin{array}{l}\text { Height } \\
\text { [mAU] }\end{array}$ & $\begin{array}{c}\text { Area } \\
\%\end{array}$ \\
\hline & & & & & & \\
\hline 1 & & BB & 7 & 5.528 & 1655. & 67.1217 \\
\hline 2 & 7264 & & & $2.70804 \mathrm{e} 4$ & 636.30121 & 32.8783 \\
\hline
\end{tabular}




\section{Decomposition study (case study):}<smiles>C=C/C=C/c1ccccc1C=O</smiles>

$6 \mathrm{~g}$ 2a (5 mol\%), TBHP (5 equiv.)

Toluene, RT<smiles>CC(C)(C)OC1OC2(CC(=O)c3ccc(I)cc3)OC1c1ccccc12</smiles>

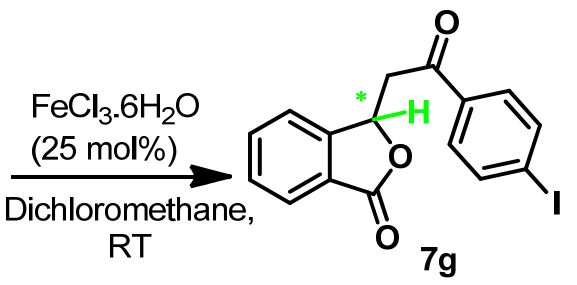

We analyzed the decomposition process of $\mathbf{7 g}$ with the help of NMR and HPLC chromatogram. From ${ }^{1} \mathrm{H}$ NMR of $\mathbf{6 g}^{\mathbf{\prime}}$ and $\mathbf{7 g}$, it's clearly visible that one proton [Red colored $(\delta 6.58)]$ is eliminated after step-II, which was present in $\mathbf{6 g}^{\mathbf{\prime}}$. In addition another one proton [Green colored $(\delta 6.0,5.8)$ ], which actually a diastereomeric $(55: 45)$ mixture of two proton is converted to single proton $\{$ Green $[\delta 6.13]$ in $7 \mathrm{~g}$. HPLC chromatogram also reflecting same result as NMR. The two diastereomers showing 55:45 ratio with (95:83) \% ee, which after decomposition giving $91 \%$ ee.

The enantiomeric ratio and $\mathrm{dr}$ of $\mathbf{6} \mathbf{g}^{\prime}$ was determined by HPLC analysis using Diacel Chiralpak OD-3 column, n-Hexane/2-Propanol = 95/05, flow rate $=1.0 \mathrm{~mL} / \mathrm{min}, \lambda=254 \mathrm{~nm}$; $t_{R}=7.5 \min$ (major), $t_{R}=8.9 \min$ (minor) for one pair and $t_{R}=18.3 \min \left(\right.$ major), $t_{R}=27.0$ $\min$ (minor) for other pair of stereoisomer.

The enantiomeric ratio of $\mathbf{7 g}$ was determined by HPLC analysis using Diacel Chiralpak OD-3 column, $\mathrm{n}$-Hexane/2-Propanol $=75 / 25$, flow rate $=1.0 \mathrm{~mL} / \mathrm{min}, \lambda=254 \mathrm{~nm} ; \mathrm{t}_{\mathrm{R}}=22.0 \mathrm{~min}$ (major), $\mathrm{t}_{\mathrm{R}}=28.7 \mathrm{~min}$ (minor). 


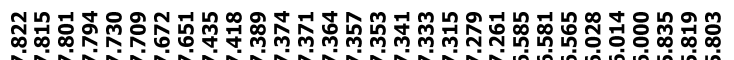

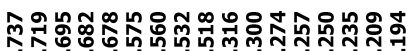

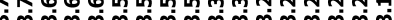

พุ๊
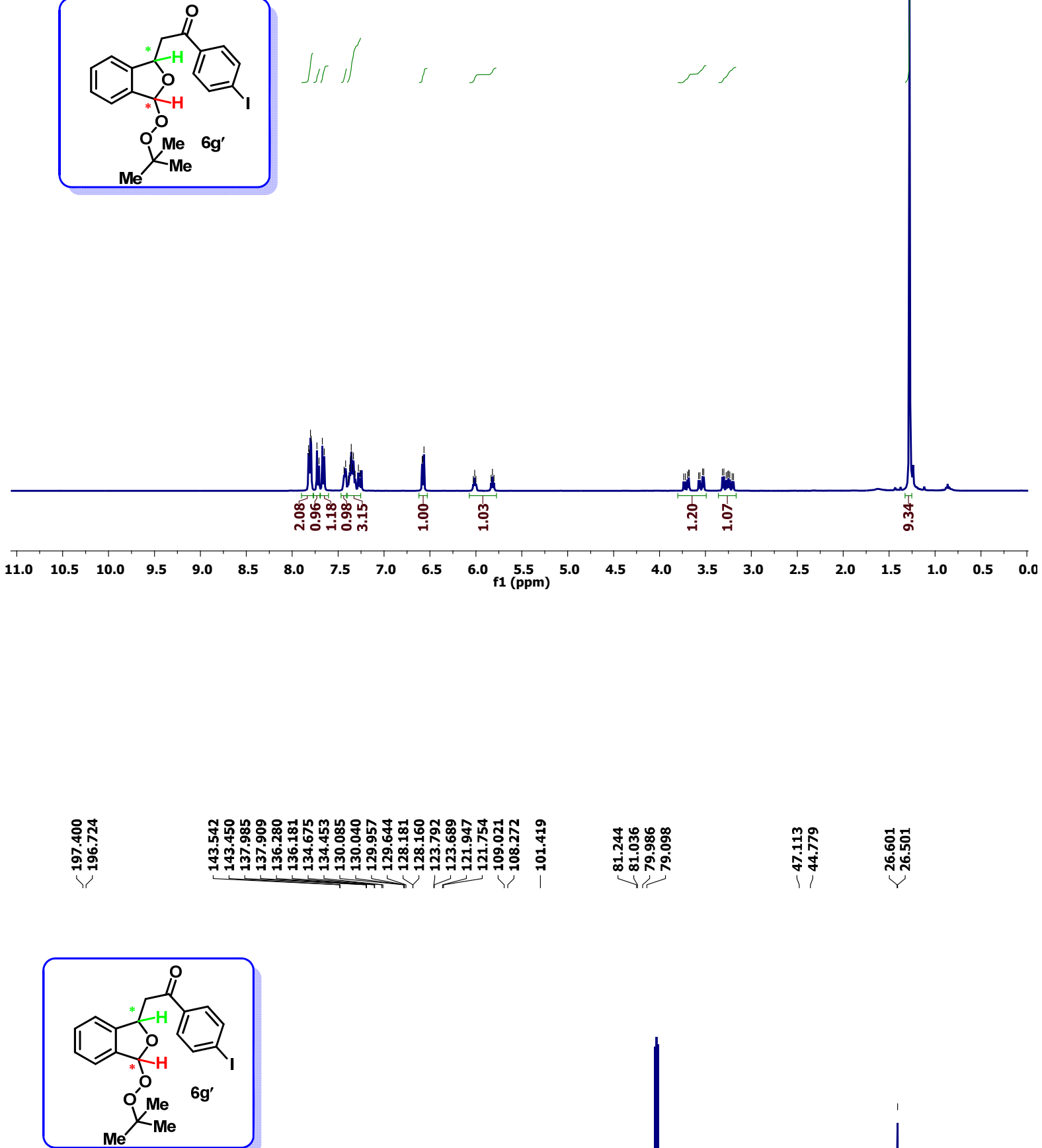


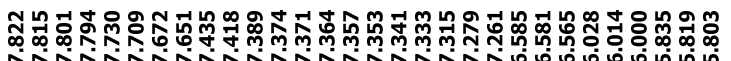

Decomposition Study
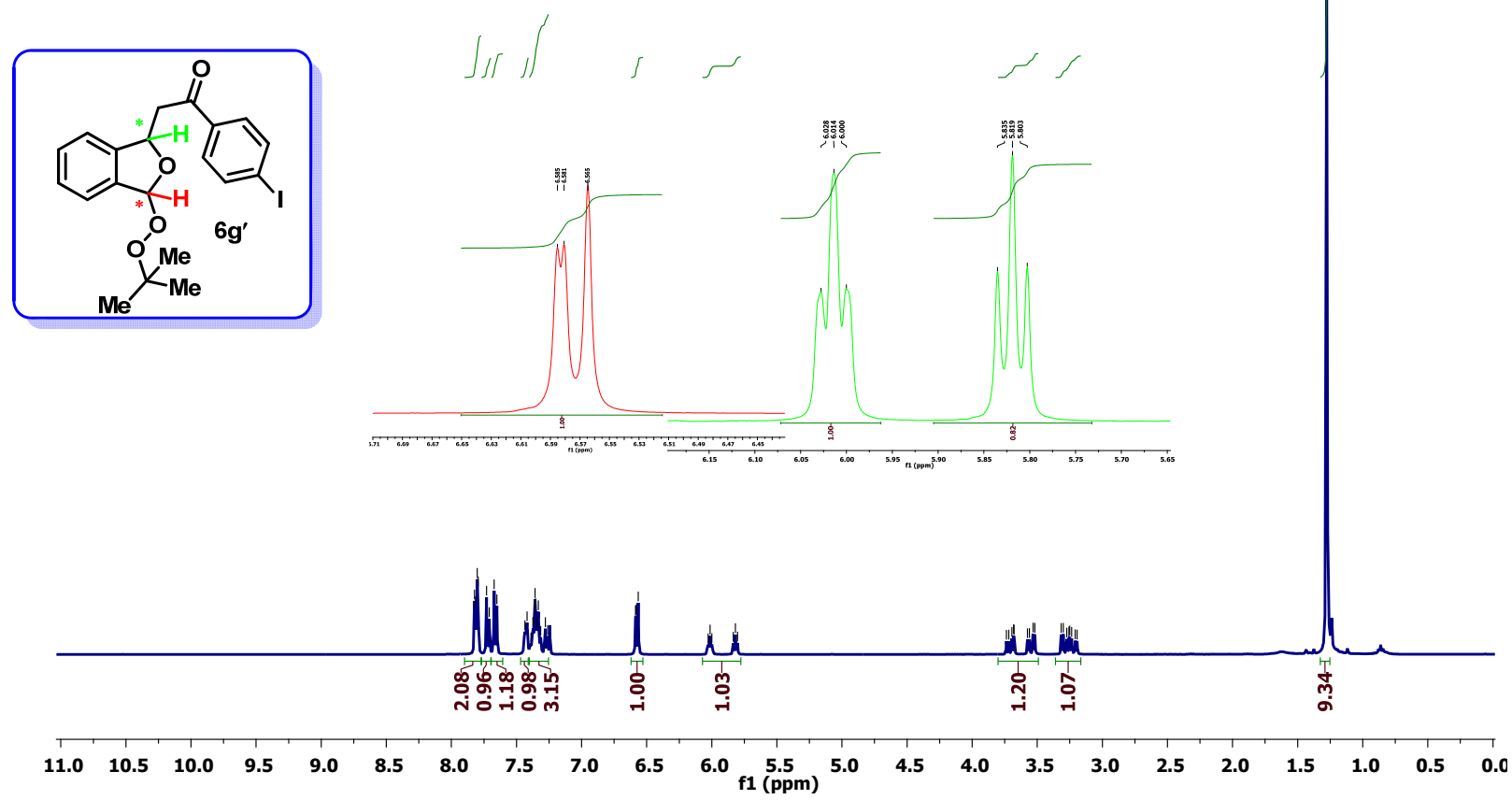

BP-154

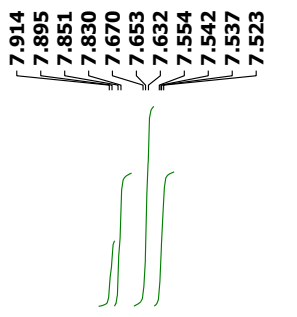

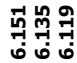

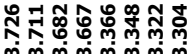

रा

mimm
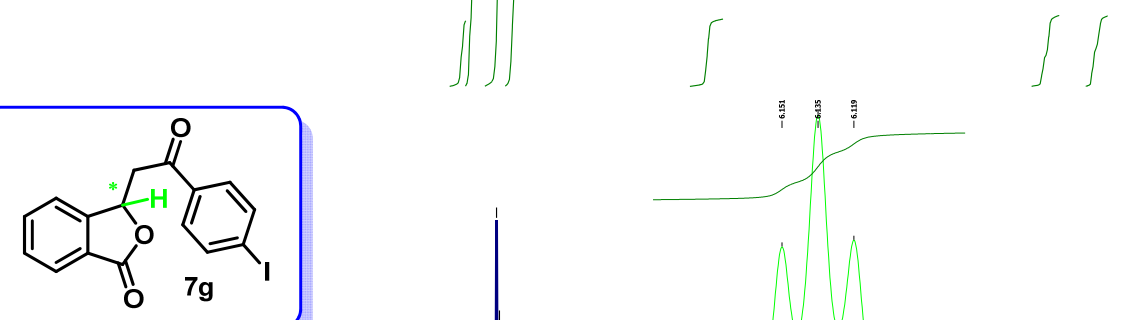

Decomposition Study

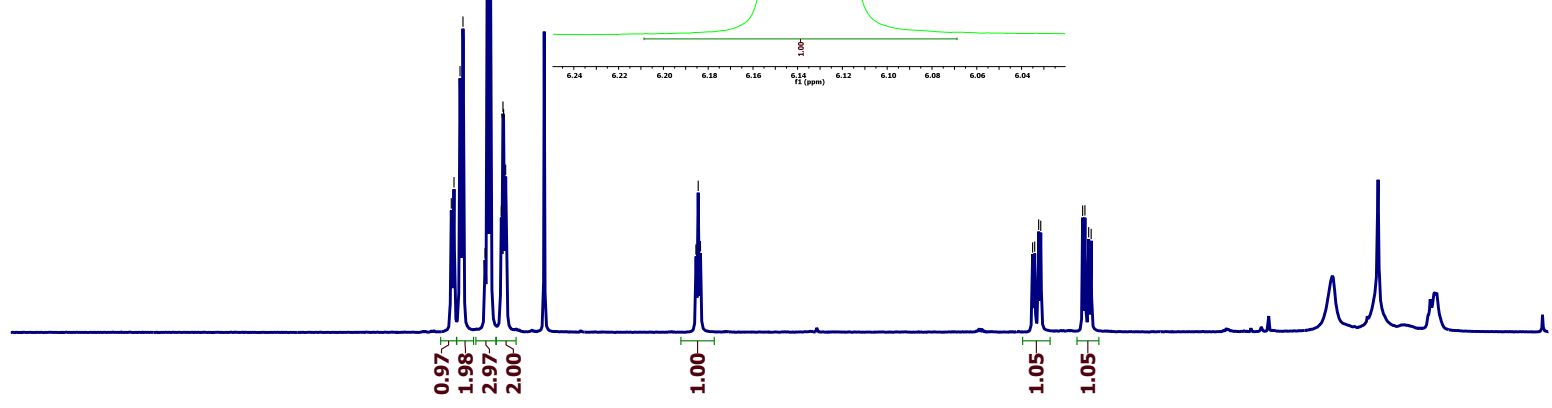

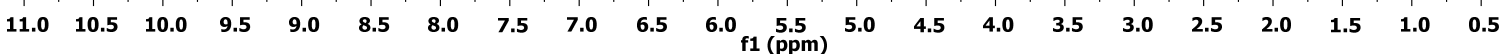



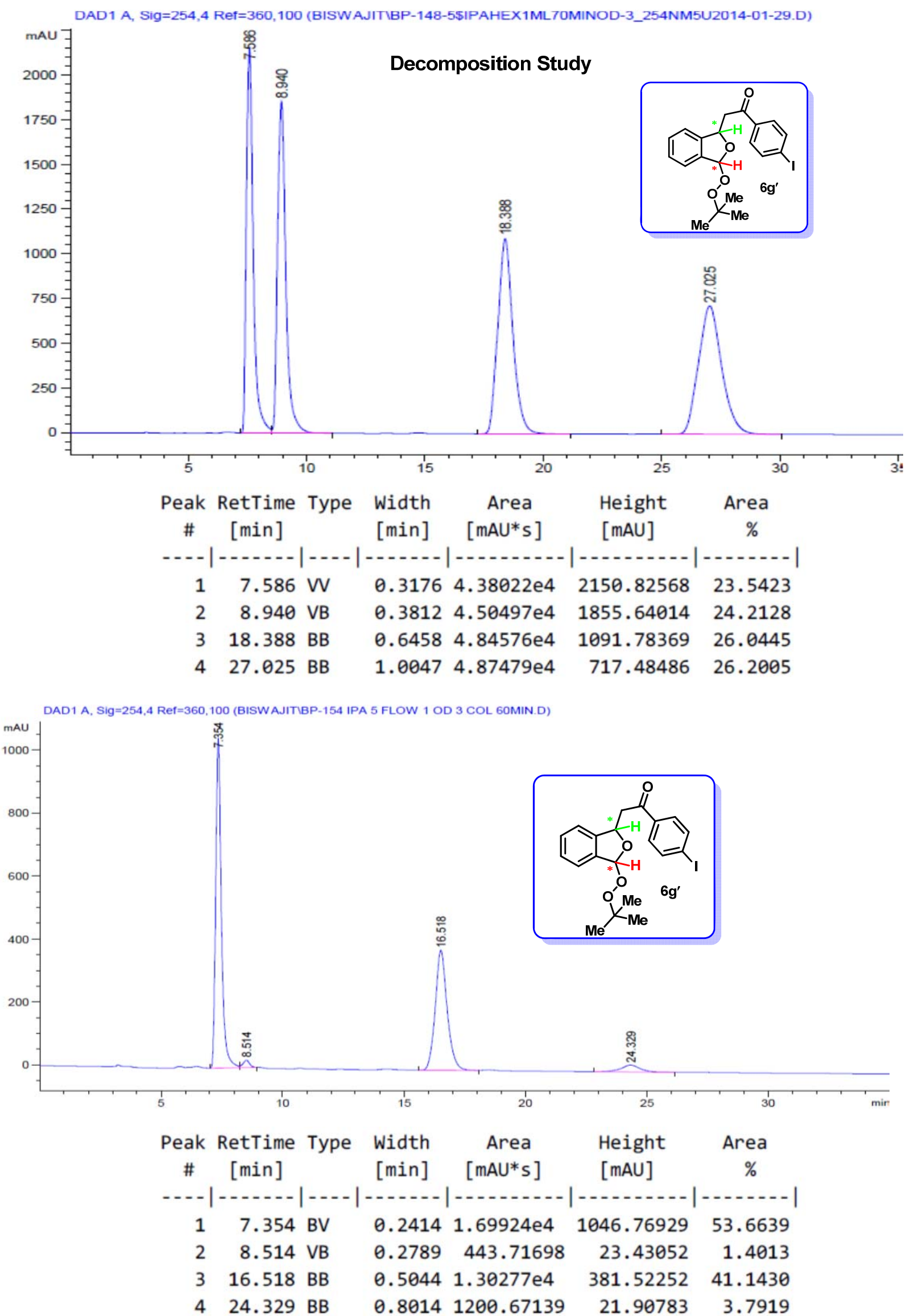

Page | 39 

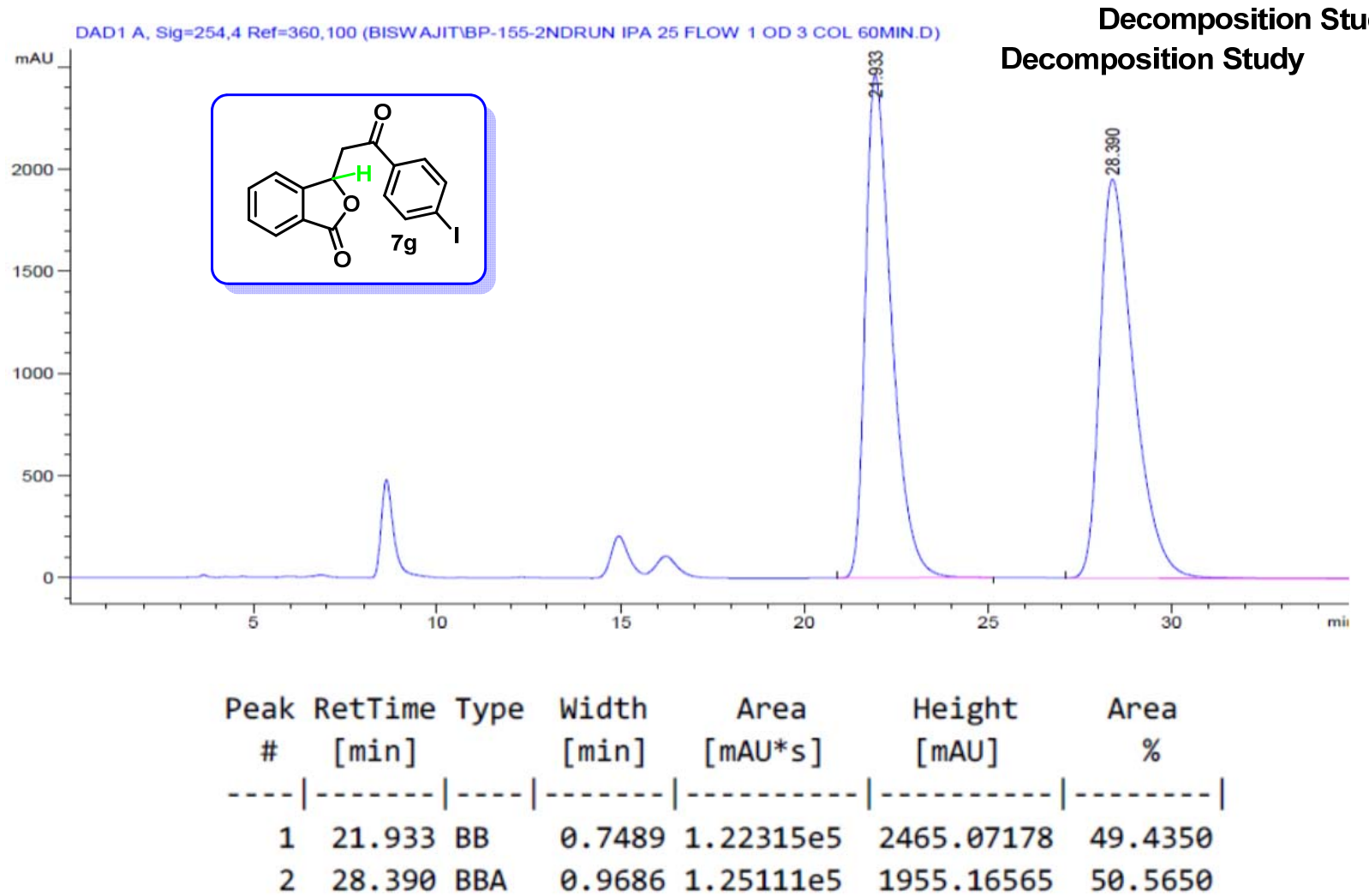

Totals : $\quad 2.47427 e 5 \quad 4420.23743$

DAD1 A, Sig=254,4 Ref=360,100 (BISWAJITIBP-154RM-2NDRUN IPA 25 FLOW 1 OD 3 COL 60MIN.D) $\mathrm{mA}$

1000

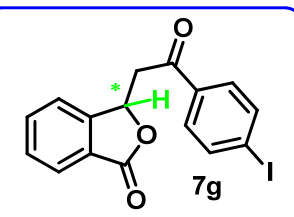

600
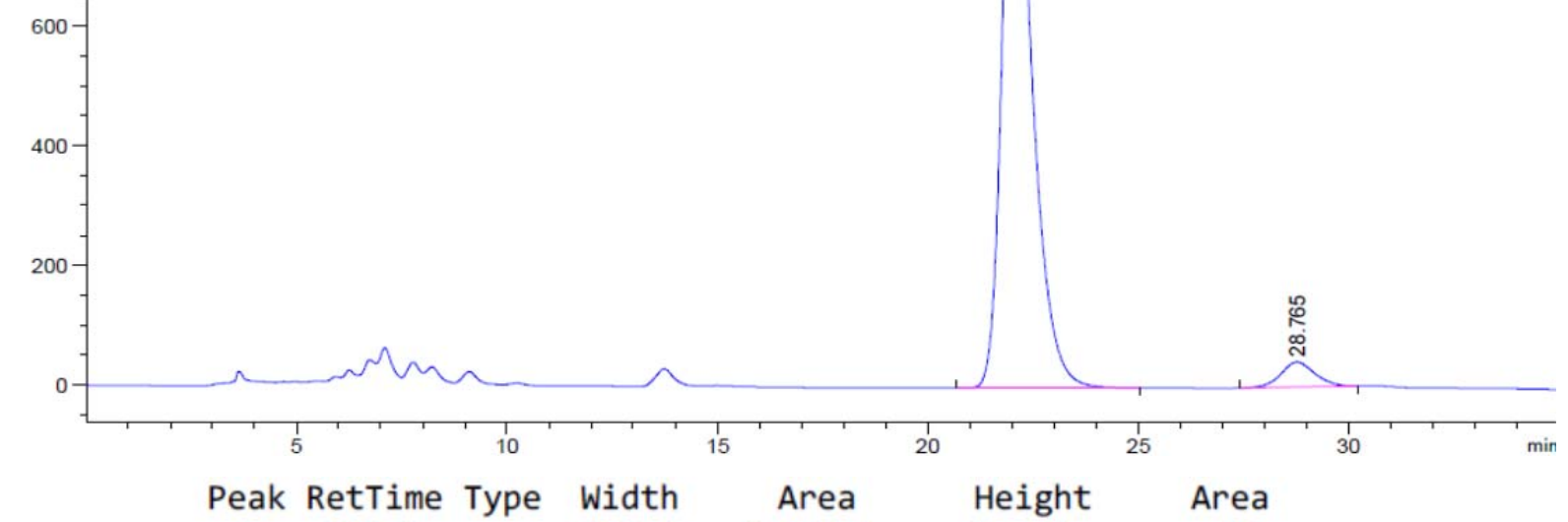

\# $\quad[\mathrm{min}] \quad[\mathrm{min}] \quad[\mathrm{mAU} * \mathrm{~s}] \quad[\mathrm{mAU}] \quad \%$

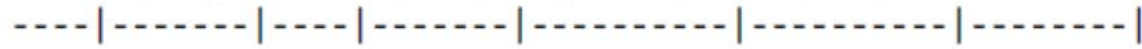

$\begin{array}{lllllll}1 & 22.041 & \text { BB } & 0.7117 & 5.21138 \mathrm{e} 4 & 1094.06934 & 95.6273\end{array}$

$\begin{array}{lllllll}2 & 28.765 & \text { BB } & 0.8507 & 2382.98389 & 42.08170 & 4.3727\end{array}$

Totals : $\quad 5.44968 \mathrm{e} 4 \quad 1136.15104$ 
Gram scale synthesis: We checked our method's potential for gram scale synthesis, which found to be excellent as well as it is. $6 \mathrm{e}(3.17 \mathrm{mmol}, 1$ gram) was tested with same reaction condition as mentioned in general procedure and provided 7e (980 mg, 93\% yield) with $86 \%$ ee and after n-hexane wash we got 7 e (670 mg, 63\% yield) with ee 93\%.
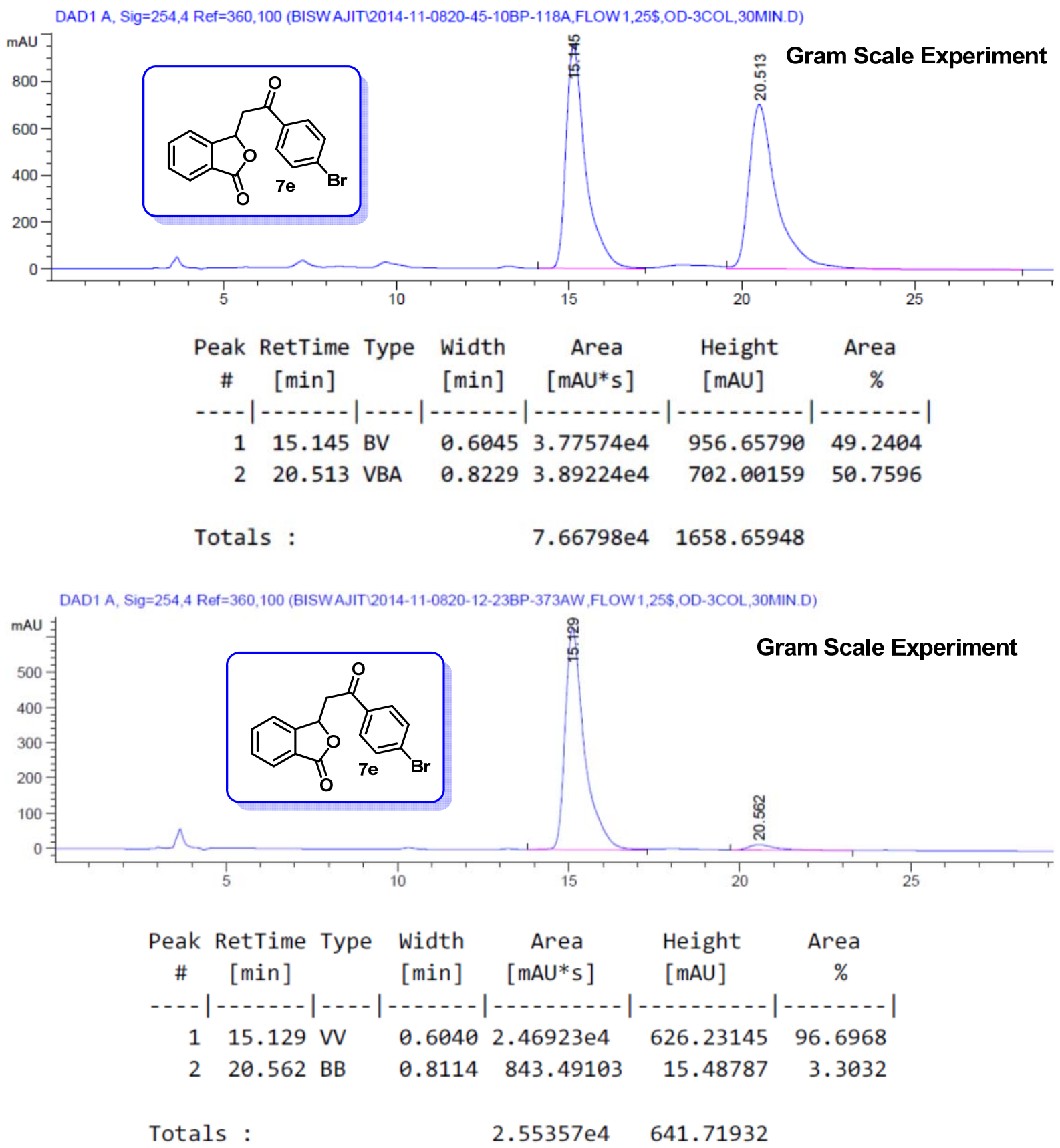


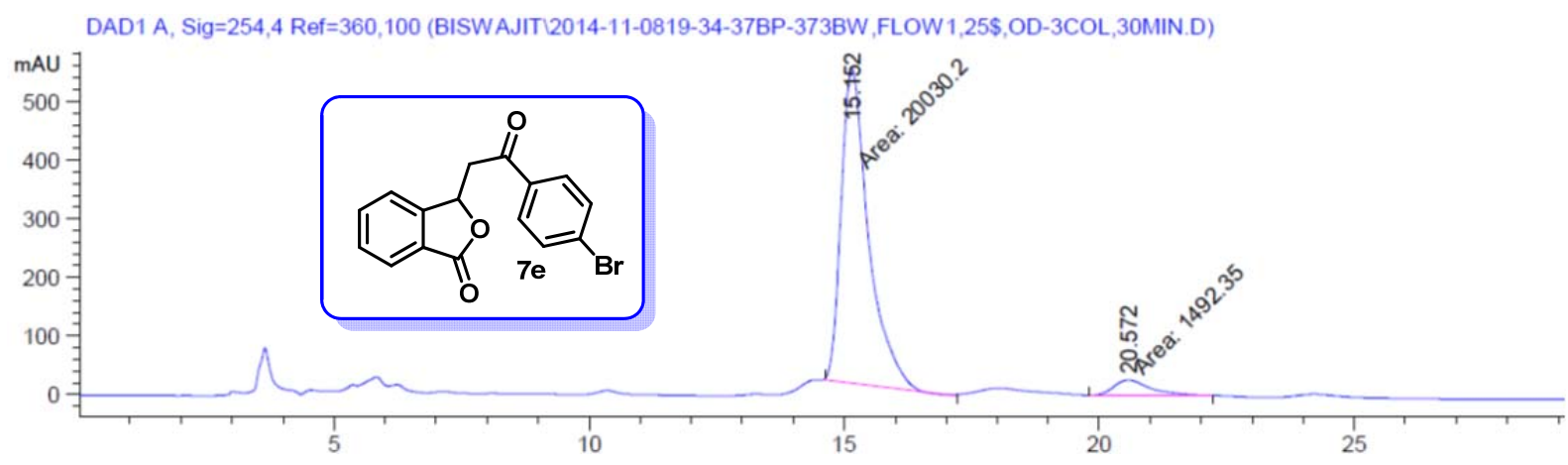

\begin{tabular}{|c|c|c|c|c|c|}
\hline $\begin{array}{c}\text { Peak } \\
\#\end{array}$ & $\begin{array}{l}\text { RetTime Type } \\
{[\min ]}\end{array}$ & $\begin{array}{l}\text { Width } \\
\text { [min] }\end{array}$ & $\begin{array}{c}\text { Area } \\
{\left[\mathrm{mAU}^{*} \mathrm{~s}\right]}\end{array}$ & $\begin{array}{l}\text { Height } \\
\text { [mAU] }\end{array}$ & $\begin{array}{c}\text { Area } \\
\%\end{array}$ \\
\hline & & & & & \\
\hline 1 & $15.152 \mathrm{MM}$ & 0.6213 & $2.00302 \mathrm{e} 4$ & 537.29193 & 93.0661 \\
\hline 2 & 20.572 MM & 0.9006 & 1492.34790 & 27.61860 & 6.9339 \\
\hline
\end{tabular}

Totals : $\quad 2.15225 \mathrm{e} 4 \quad 564.91053$

Determination of absolute configuration: In our previous report, ${ }^{1}$ we reported the configuration of 1,3-dihydroisobenzofurans by converting it to TBDMS protected alcohol which was compared with the literature optical rotation $[(-)-(\mathrm{S})]^{1}$. The configuration was found to be R. We compared the HPLC chromatogram of previously ${ }^{1}$ oxidized 2-(1,3dihydroisobenzofuran-1-yl)-1-phenylethanone with this present phthalide 7a by which the configuration was found to be same $(\mathrm{R})$ and optical rotation was also found to be same. 

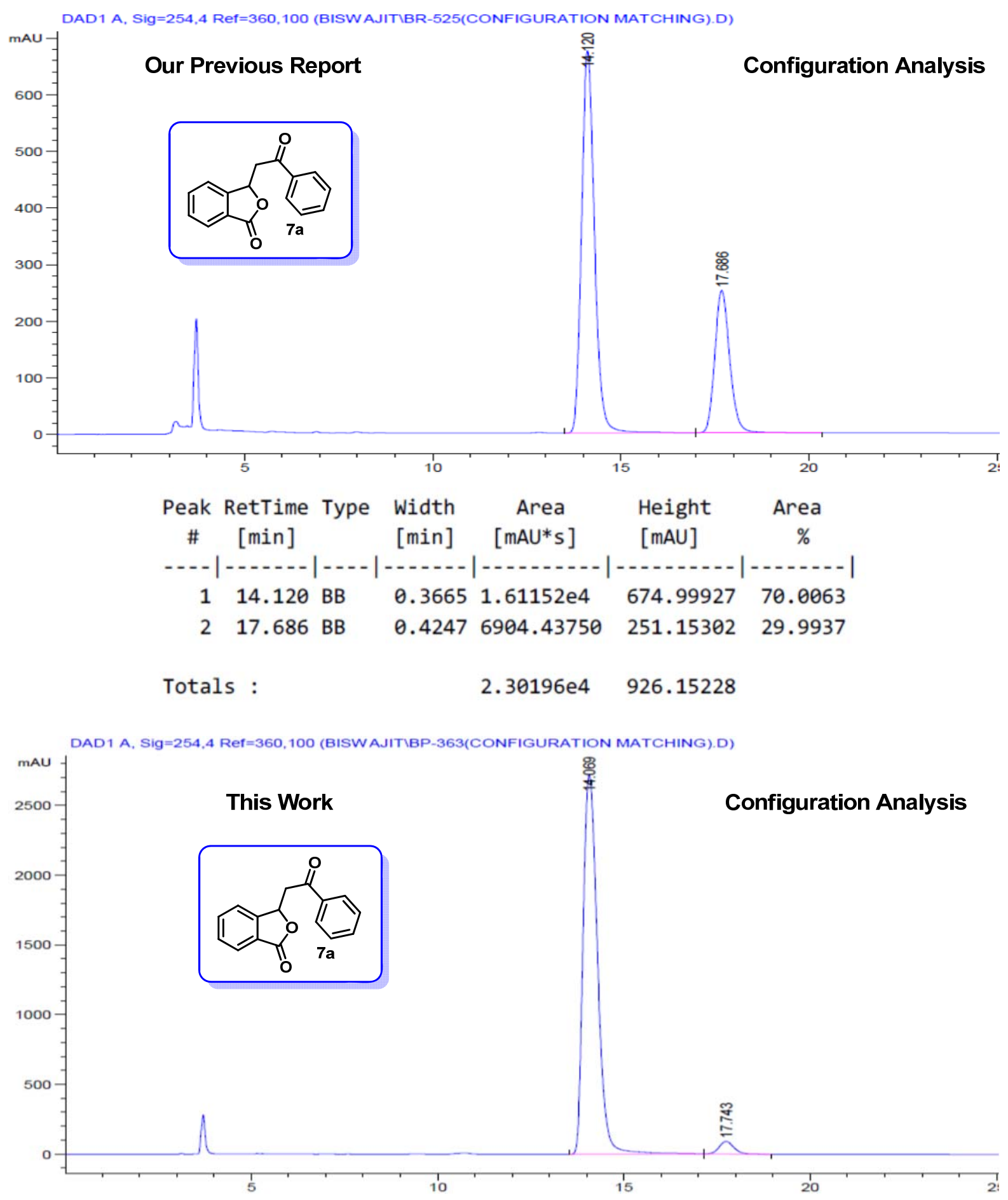

\begin{tabular}{|c|c|c|c|c|c|c|}
\hline $\begin{array}{c}\text { Peak } \\
\quad \#\end{array}$ & $\begin{array}{l}\text { RetTime } \\
\text { [min] }\end{array}$ & Type & $\begin{array}{l}\text { Width } \\
\text { [min] }\end{array}$ & $\begin{array}{c}\text { Area } \\
{\left[\mathrm{mAU}^{*} \mathrm{~s}\right]}\end{array}$ & $\begin{array}{l}\text { Height } \\
{[\mathrm{mAU}]}\end{array}$ & $\begin{array}{c}\text { Area } \\
\%\end{array}$ \\
\hline-1 & & & & - & | & -----1 \\
\hline 1 & 14.069 & BB & 0.4214 & $7.30522 \mathrm{e} 4$ & 2719.12134 & 96.7763 \\
\hline 2 & 17.743 & BB & 0.4221 & 2433.45703 & 89.24718 & 3.2237 \\
\hline ta & & & & 7.54857e4 & 2808.36852 & \\
\hline
\end{tabular}




\section{References:}

1. Barnala, R.; Das, B. G.; Ghorai, P. Org. Lett. 2014, 16, 5580.

2. Barnala, R.; Maity, S.; Das, B. G.; Ghorai, P. J. Org. Chem. 2015, 80, 7008.

3. Maity, S.; Parhi, B.; Ghorai, P. Angew. Chem., Int. Ed. (DOI: 10.1002/anie.201511165)

4. Asano, K.; Matsubara, S. J. Am. Chem. Soc. 2011, 133, 16711. 